\title{
Minimal Walks and Applications
}

\author{
Rafael de Lima Bordoni
}

\author{
DISSERTAÇÃO APRESENTADA \\ $\mathrm{AO}$ \\ Instituto de MATEMÁticA E EstatísticA \\ DA \\ Universidade DE SÃO PAULO \\ PARA \\ OBTENÇÃO DO TÍTULO \\ DE \\ MESTRE EM CiÊNCIAS
}

\author{
Programa: Mestrado em Matemática \\ Orientador: Prof. Dr. Artur Hideyuki Tomita
}

Durante o desenvolvimento deste trabalho o autor recebeu auxílio financeiro da CNPq

São Paulo, outubro de 2018 


\section{Minimal Walks and Applications}

Esta versão da dissertação contém as correções e alterações sugeridas pela Comissão Julgadora durante a defesa da versão original do trabalho, realizada em 10/08/2018. Uma cópia da versão original está disponível no

Instituto de Matemática e Estatística da Universidade de São Paulo.

Comissão Julgadora:

- Prof. Dr. Artur Hideyuki Tomita (orientador) - IME-USP

- Prof ${ }^{a}$. Dr ${ }^{\mathrm{a}}$. Ana Carolina Boero - UFABC

- Prof. Dr. Leandro F. Aurichi - ICMC-USP 


\section{Agradecimentos}

Em primeiro lugar, eu agradeço a minha família e amigos. A meu irmão Ramon, meus pais Maria Aparecida e Cairo e a minha sobrinha Laura, que acabou de nascer e eu já a amo. Mesmo que vocês estejam longe, saibam que eu nunca esqueci de vocês. Em nenhum segundo. Aos meus amigos Marli, Marisa, Ana e Luiz, que sempre estiveram presente e me fizeram companhia. Saibam todos vocês aqui mencionados que por mais que eu não demonstre, não quer dizer que eu não sinta. Só porque eu guardo meus sentimentos pra mim não quer dizer que eles sejam menos intensos. Eu posso não dizer, mas eu os amo muito.

Em segundo lugar, a meu orientador Artur Tomita, que sempre foi paciente, compreensivo e respeitou o meu ritmo. Ao $\mathrm{CNPq}$, cujo o apoio foi fundamental a esse projeto. Sem esse apoio, nada disso seria possível.

Em terceiro lugar, a todos os meus professores da escola que fizeram a diferença em minha vida. Professores Cel. Gaeta, Bomfim, René e Edmilson, obrigado por acreditarem em mim. Vocês me inspiraram e me mostraram todas as possibilidades que eu possuo. Muito obrigado por me mostrarem do que somos capazes.

Por fim, quero agradecer a OBMEP, que tudo começou. Me botou no caminho dessas pessoas incríveis e iniciou essa corrente de eventos que me fez quem sou hoje.

A todos e a todas, do fundo do meu coração, muito obrigado. 


\section{Resumo}

BORDOnI, R. L. Passeios Mínimos e Aplicações. 2018. 86 f. Dissertação (Mestrado) Instituto de Matemática e Estatística, Universidade de São Paulo, São Paulo, 2018.

Passeios mínimos são um método de demonstrações em teoria dos conjuntos e topologia geral. A ideia foi inventada por Stevo Todorcevic e é profundamente explicada em seu livro [Tod07]. Apesar do trabalho principal dessa dissertação ser a existência do L espaço em [Moo06], a intenção é explicar os fundamentos dos passeios mínimos um tanto detalhadamente aqui. Não apenas os fundamentos, mas algumas outras aplicações também. Além disso, foi incluso um extenso apêndice para cobrir o básico de lógica, teoria dos modelos e teoria dos conjuntos necessárias para o entendendimento desse trabalho da forma mais didática possível, com a intenção de torna-lo acessível a qualquer estudante de graduação.

Palavras-chave: topologia geral, teoria dos conjuntos, teoria dos modelos. 


\section{Abstract}

BORDONI, R. L. Minimal Walks and Applications. 2018. 86 f. Dissertação (Mestrado) - Instituto de Matemática e Estatística, Universidade de São Paulo, São Paulo, 2018.

Minimal Walks are a method of demonstrations in set theory and general topology. The idea was first brought by Stevo Todorcevic, and is deeply explained in [Tod07]. Although the main work of this document will be the construction of the L space in [Moo06], we intend to explain walk's fundamentals in a bit more detail here. Not only they are the foundation of Moore's work, we also intend to show more of their applications. Aside from that, we included an extensive appendix to cover the basics of logic, model theory and set theory necessary to understanding this work in the most didactic way, with the intention to make it accessible to any undergraduate student.

Keywords: general topology, set theory, model theory. 


\section{Contents}

$\begin{array}{ll}\text { Introduction } & 1\end{array}$

1 Minimal Walks $\quad 3$

1.1 Trace functions . . . . . . . . . . . . . . . . . . . . 3

1.2 Weight functions and coherent sequences . . . . . . . . . . . . 7

1.3 Full lower trace . . . . . . . . . . . . . . . . . . . . . . . . . 10

2 Countable Elementary Submodels $\quad 13$

2.1 Basics . . . . . . . . . . . . . . . . . . . . . . . 13

2.2 Useful facts . . . . . . . . . . . . . . . . . . . . . . 16

3 The L Space $r$

3.1 Oscillations of the lower trace . . . . . . . . . . . . . . . . . 19

3.2 The $o$ function . . . . . . . . . . . . . . . . . . . 25

3.3 The construction . . . . . . . . . . . . . . . . 27

4 Applications in Model Theory 33

4.1 Oscillations on the upper trace . . . . . . . . . . . . . . . . . 33

4.2 Results . . . . . . . . . . . . . . . . . . . . 37

A Mathematical Logic $\quad 39$

A.1 Languages . . . . . . . . . . . . . . . . . . . . . . . . . . . . . . 39

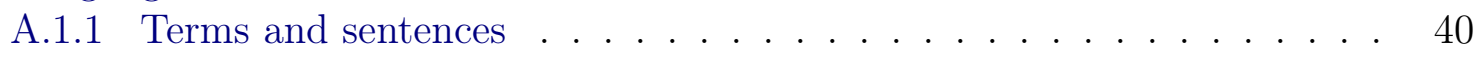

A.1.2 Formulas . . . . . . . . . . . . . . . . . . . 43

A.2 Logics . . . . . . . . . . . . . . . . . . . . . 46

A.2.1 Propositional logic . . . . . . . . . . . . . . . . . . . 47

A.2.2 First order logic . . . . . . . . . . . . . . . . . . 56

A.3 Theories . . . . . . . . . . . . . . . . . . 60

A.3.1 Initial concepts . . . . . . . . . . . . . . . . 61

A.3.2 Results . . . . . . . . . . . . . . . . . 63

A.4 Models . . . . . . . . . . . . . . . . . . . . 65

$\begin{array}{ll}\text { B Set Theory } & \mathbf{7 1}\end{array}$

B.1 Axioms of ZFC . . . . . . . . . . . . . . . . . 71

B.2 Ordinals and cardinals . . . . . . . . . . . . . . . . . . . . . . . . . . . . . . . . . . . . . . . . .

B.3 Sets of hereditary cardinality . . . . . . . . . . . . . . 89

$\begin{array}{ll}\text { Bibliography } & 93\end{array}$ 


\section{Introduction}

At it's core, walks let us construct mathematical structures to fulfill a certain role. It can be a sequence, a function, some linearly ordered set or just any structure with a given property we need. Todorcevic used it for the first time in [Tod87] and later this technique showed it's promise by having many applications in both topology and combinatorial set theory. In 2006, Moore used this technique to construct the L space.

This construction will be the main result showcased in this document, so we are going to talk about the history of this problem first. Let's start with some definitions.

Definition 0.0.1. Let $X$ be a regular Hausdorff topological space. We say that $X$ is an $\mathrm{L}$ space if $X$ is hereditarily Lindelöf but not hereditarily separable. We say that $X$ is an $\mathrm{S}$ space if $X$ is hereditarily separable but not hereditarily Lindelöf.

For many years, they were considered duals and mathematicians tried to understand them in conjunction. It was believed that any model of set theory that contained an S space would also contain an L space and vice versa. The following theorem on [Zen80] helped solidifying that belief.

Theorem 0.0.2. Let $X$ and $Y$ be completely regular spaces. If $Y$ is second countable, $X^{n}$ is hereditarily Lindelöf for all $n<\omega$ if and only if $C_{p}(X, Y)$ is hereditarily separable. Also, $X^{n}$ is hereditarily separable for all $n<\omega$ if and only if $C_{p}(X, Y)$ is hereditarily Lindelöf.

We call $C_{p}(X, Y)$ the set of all continuous functions $f: X \longrightarrow Y$ as a topological subset of $Y^{X}$. Later on, Todorcevic proved under PFA that there are no S spaces.

Theorem 0.0.3. Let $X$ be a regular Hausdorff space. Assuming PFA, if $X$ is hereditarily separable, then $X$ is hereditarily Lindelöf.

It was only a decade ago that Moore proved that $\mathrm{L}$ spaces do in fact exist under ZFC with no additional axioms in [Moo06]. This will be the main result of this document. 
Regardless, Todorcevic's technique was also applied to many other problems other than solving the existence of S and L spaces. Todorcevic himself was the one who used it to construct many things from models and topological spaces, as the creator of the technique. For instance, he used walks in model theory, as showcased in [Tod07] and the last application of this document, which is...

Theorem 0.0.4. A model $M$ of set theory which correctly understands $\omega_{1}$ also contains a partition of $\omega_{1}$ into infinitely many pairwise-disjoint subsets that are stationary in the class $V$ of sets.

This result uses a construction similar to that of the $\mathrm{L}$ space, with an analogous object that generates similar properties but applied to very different places. This goes to show that walks can have a wide range of applications. Even then, these two similar objects are just the tip of the iceberg. On [Tod07], Todorcevic defines other objects with different properties and proves more results, mostly on set theory and combinatorics. 


\section{Chapter 1}

\section{Minimal Walks}

So, let's get started. First, we need to define the places where we take the steps of each walk.

Definition 1.0.1. Given a countable ordinal $\alpha$, let $C_{\alpha}=\left\{c_{\alpha}(n): n<\omega\right\}$ be a cofinal sequence in $\alpha$. A C-sequence is a set $\left\{C_{\alpha}: \alpha<\omega_{1}\right\}$ of such sets. It's easier to assume that $C_{\alpha+1}=\{\alpha\}$ for every $\alpha<\omega_{1}$ and that $C_{\alpha}$ is crescent when $\alpha$ is limit.

Note that since the order type of $C_{\alpha}$ is $\omega$, then there are only finite many ordinals smaller than a given $\gamma<\alpha$ in $C_{\alpha}$. The idea is that we use the C-sequences to build ladders for traversing $\omega_{1}$ from one ordinal to a smaller one. Throughout the rest of this document, we will assume that we have some fixed C-sequence.

\subsection{Trace functions}

Definition 1.1.1. Given $\alpha \leq \beta$ countable ordinals, we recursively define the upper trace $\operatorname{Tr}(\alpha, \beta)$ by:

- $\operatorname{Tr}(\alpha, \alpha)=\{\alpha\}$

- $\operatorname{Tr}(\alpha, \beta)=\operatorname{Tr}\left(\alpha, \min \left(C_{\beta} \backslash \alpha\right)\right) \cup\{\beta\}$.

Alternatively, we can define it by the finite set of ordinals $\operatorname{Tr}(\alpha, \beta)=\left\{\beta_{0}, \cdots, \beta_{n}\right\}$ such that $\beta=\beta_{0}>\beta_{1}>\cdots>\beta_{n-1}>\beta_{n}=\alpha$ and $\beta_{i+1}=\min \left(C_{\beta_{i}} \backslash \alpha\right)$. This last definition 
is easier to understand. This set represents the steps taken from $\beta$ to $\alpha$ in what we call a minimal walk.

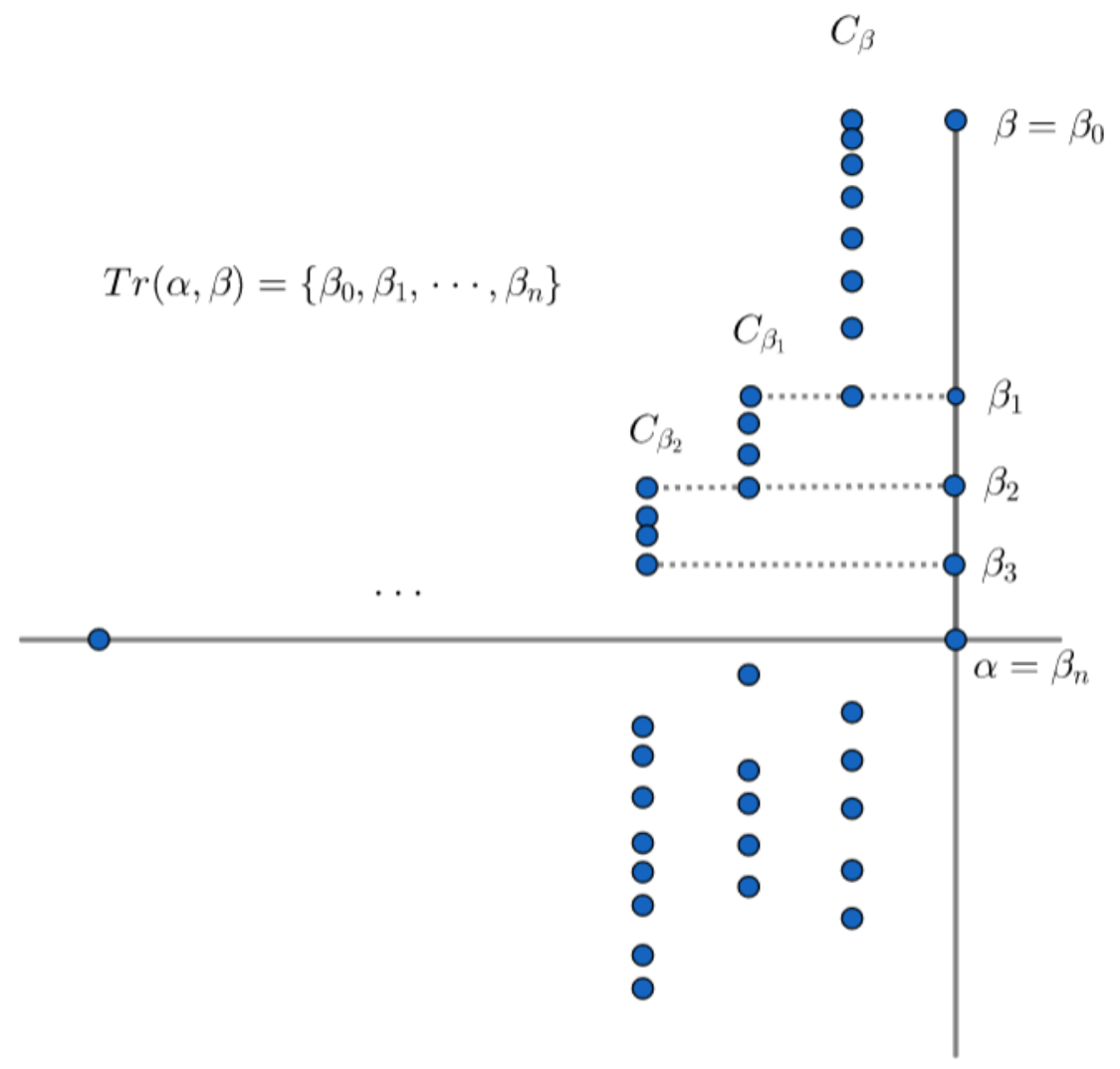

Definition 1.1.2. Given $\alpha \leq \beta$ countable ordinals, we recursively define the lower trace $\mathrm{L}(\alpha, \beta)$ by:

- $\mathrm{L}(\alpha, \alpha)=\varnothing$

- $\mathrm{L}(\alpha, \beta)=\mathrm{L}\left(\alpha, \min \left(C_{\beta} \backslash \alpha\right)\right) \cup\left\{\max \left(C_{\beta} \cap \alpha\right)\right\} \backslash \max \left(C_{\beta} \cap \alpha\right)$.

Alternatively, we can define it by the finite set of ordinals $\mathrm{L}(\alpha, \beta)=\left\{\xi_{0}, \cdots, \xi_{n-1}\right\}$ such that $\xi_{i}=\max \cup\left\{C_{\beta_{0}} \cap \alpha, \cdots, C_{\beta_{i}} \cap \alpha\right\}$, where the $\beta_{j}$ 's are the steps in $\operatorname{Tr}(\alpha, \beta)$. This is possible because each $C_{\beta_{j}} \cap \alpha$ is finite as noted earlier and there are finite many of these sets in reunion, so we can take their maximum point. We can also note that they are not necessarily all different, but at least each one is not greater than the next, so we have that $\xi_{0} \leq \xi_{1} \leq \cdots \leq \xi_{n}<\alpha$. This means that in the end, we have $\mathrm{L}(\alpha, \beta)<\operatorname{Tr}(\alpha, \beta)$, with both $\alpha$ and $\beta$ being the minimum and maximum of $\operatorname{Tr}(\alpha, \beta)$ and that $|\mathrm{L}(\alpha, \beta)|<|\operatorname{Tr}(\alpha, \beta)|$.

So, now we have $\operatorname{Tr}(\alpha, \beta)$ going from $\beta$ down to $\alpha$ and $\mathrm{L}(\alpha, \beta)$ going up, getting closer to $\alpha$. Next, we will talk about some properties. 


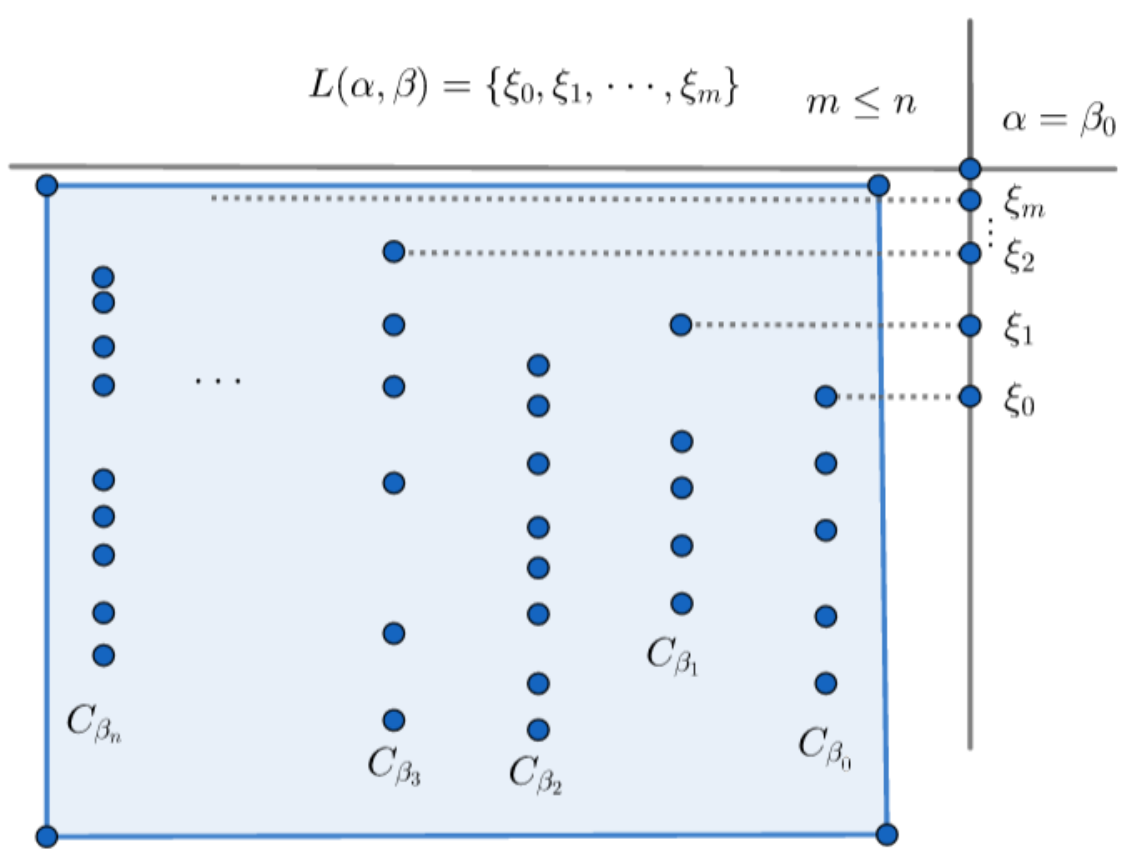

Proposition 1.1.3. Given countable ordinals $\alpha<\beta<\gamma$ such that $\mathrm{L}(\beta, \gamma)<\mathrm{L}(\alpha, \beta)$, then

(i) If $\mathrm{L}(\beta, \gamma)<\alpha$, then $\operatorname{Tr}(\alpha, \gamma)=\operatorname{Tr}(\alpha, \beta) \cup \operatorname{Tr}(\beta, \gamma)$;

(ii) If $\mathrm{L}(\beta, \gamma)<\mathrm{L}(\alpha, \beta)$, then $\mathrm{L}(\alpha, \gamma)=\mathrm{L}(\alpha, \beta) \cup \mathrm{L}(\beta, \gamma)$.

When we say that a set of ordinals $A$ is lesser than another set of ordinals $B$, we mean that all $x \in A$ are lesser than each $y \in B$. We will use this notation from now on constantly. Now, let's prove this proposition.

Proof. $\mathrm{L}(\beta, \gamma)<\alpha$ implies that $C_{\zeta} \cap \alpha=C_{\zeta} \cap \beta$ whenever $\zeta \in \operatorname{Tr}(\beta, \gamma)$ because if there were $\zeta_{0} \in \operatorname{Tr}(\beta, \gamma)$ such that $C_{\zeta_{0}} \cap \alpha \subset C_{\zeta_{0}} \cap \beta$, then there would be $x \in C_{\zeta_{0}} \cap \beta$ greater than or equal to $\alpha$ and since $\mathrm{L}(\beta, \gamma)=\left\{\xi_{0}, \cdots, \xi_{k}\right\}$ with $\xi_{i}=\max \bigcup\left\{C_{\beta_{j}} \cap \beta: 0 \leq j \leq i\right\}$, then $x$ or something greater than $x$ would be in $\mathrm{L}(\beta, \gamma)$, which means that $\mathrm{L}(\beta, \gamma) \nless \alpha$.

With that, we have that $\beta \in \operatorname{Tr}(\alpha, \gamma)$ because

$$
C_{\zeta} \cap \alpha=C_{\zeta} \cap \beta \Longrightarrow C_{\zeta} \backslash \alpha=C_{\zeta} \backslash \beta \Longrightarrow \min \left(C_{\zeta} \backslash \alpha\right)=\min \left(C_{\zeta} \backslash \beta\right)
$$


whenever $\zeta \in \operatorname{Tr}(\beta, \gamma)$. This means, by the alternate definition of the upper trace, that $\operatorname{Tr}(\alpha, \gamma)=\operatorname{Tr}(\alpha, \beta) \cup \operatorname{Tr}(\beta, \gamma)$, finishing the first part of this proof.

For the second part, we need to recall that $\mathrm{L}(\alpha, \beta)<\alpha$ for all $\alpha<\beta$, so $\mathrm{L}(\beta, \gamma)<$ $\mathrm{L}(\alpha, \beta)$ implies that $\mathrm{L}(\beta, \gamma)<\alpha$, therefore we already have that $\operatorname{Tr}(\alpha, \gamma)=\operatorname{Tr}(\alpha, \beta) \cup$ $\operatorname{Tr}(\beta, \gamma)$. With that in mind, let's denote $\operatorname{Tr}(\alpha, \gamma)$ by

$$
\operatorname{Tr}(\alpha, \gamma)=\left\{\gamma_{0}, \gamma_{1}, \cdots, \gamma_{n_{0}}, \cdots, \gamma_{n-1}, \gamma_{n}\right\}
$$

with $\gamma_{0}=\gamma, \gamma_{n_{0}}=\beta$ and $\gamma_{n}=\alpha$. This way, with the alternate definition of the lower trace in mind, we can write $\mathrm{L}(\alpha, \gamma)=\left\{\xi_{0}, \cdots, \xi_{n-1}\right\}$ with each $\xi_{i}$ as $\max \bigcup\left\{C_{\gamma_{j}} \cap \alpha: 0 \leq\right.$ $j \leq i\}$. If $i<n_{0}$, then $C_{\gamma_{i}} \cap \alpha=C_{\gamma_{i}} \cap \beta$ and therefore $\mathrm{L}\left(\beta_{\gamma}\right)=\left\{\xi_{0}, \cdots, \xi_{n_{0}-1}\right\}$. On the other hand, $\max \left(C_{\gamma_{n_{0}}} \cap \alpha\right)>\xi_{l_{0}-1}$ because it is the first element of $\mathrm{L}(\alpha, \beta)$ and $\mathrm{L}(\beta, \gamma)<\mathrm{L}(\alpha, \beta)$. This means that if $i \geq n_{0}$,

$$
\max \bigcup_{j=0}^{i}\left(C_{\gamma_{j} \cap \alpha}\right)=\max \bigcup_{j=n_{0}}^{i}\left(C_{\gamma_{j} \cap \alpha}\right) .
$$

This means that $\mathrm{L}(\alpha, \beta)=\left\{\xi_{n_{0}}, \xi_{n_{0}+1}, \cdots, \xi_{n-1}\right\}$, which ends our proof.

Hopefuly, this gives some insight on how walks behave. Even though the definition seems arbitrary, they are designed to walk together if they step on the same ordinal along their way. This next proposition is also pretty easy to prove, but will be used quite a lot in this document.

Proposition 1.1.4. Let $\alpha<\beta$ be countable ordinals greater than zero such that $\beta$ is limit. Then

$$
\lim _{\alpha \rightarrow \beta} \min \mathrm{L}(\alpha, \beta)=\beta
$$

Proof. By the alternate definition, we know that $\mathrm{L}(\alpha, \beta)=\left\{\xi_{0}, \cdots, \xi_{n-1}\right\}$ where each $\xi_{i}$ is equal to $\max \bigcup\left\{C_{\beta_{j}} \cap \alpha: 0 \leq j \leq i\right\}$, which means that $\xi_{0}=\min \mathrm{L}(\alpha, \beta)$, since $\xi_{0} \leq \cdots \leq$ $\xi_{n-1}$. So we have

$$
\lim _{\alpha \rightarrow \beta} \min \mathrm{L}(\alpha, \beta)=\lim _{\alpha \rightarrow \beta} \max C_{\beta_{0}} \cap \alpha .
$$

Since $\beta_{0}=\beta$ and $\beta$ is limit, then $C_{\beta}$ is a sequence converging to $\beta$, meaning that it's limit is $\beta$. 


\subsection{Weight functions and coherent sequences}

This next section will be about four new functions linked to the upper trace. We will go a little deeper now.

Definition 1.2.1. Given $\alpha<\beta$ countable ordinals and $\operatorname{Tr}(\alpha, \beta)=\left\{\beta_{0}, \cdots, \beta_{n}\right\}$, we call the natural number $\left|C_{\beta_{i}} \cap \alpha\right|$ the weight of $\beta_{i}$ for all $i<n$.

Notice that we had to leave $\beta_{n}$ out because $\beta_{n}=\alpha$ means it's weight is infinite.

Definition 1.2.2. We define the full code of the walk as a finite sequence $\varrho_{0}(\alpha, \beta)$, recursively constructed by:

- $\varrho_{0}(\alpha, \alpha)=\varnothing$

- $\varrho_{0}(\alpha, \beta)=\left\langle\left|C_{\beta} \cap \alpha\right|\right\rangle \wedge \varrho_{0}\left(\alpha, \min \left(C_{\beta} \backslash \alpha\right)\right)$,

where the symbol $\wedge$ means concatenating the sequences in that order.

Alternatively, given $\operatorname{Tr}(\alpha, \beta)=\left\{\beta_{0}, \cdots, \beta_{n}\right\}$, we have that $\varrho_{0}(\alpha, \beta)=\left\langle\left|C_{\beta_{0}} \cap \alpha\right|, \cdots,\right| C_{\beta_{n-1}} \cap$ $\alpha|\rangle$. The full code is the sequence formed by the weights of the steps in the walk from $\beta$ to $\alpha$ in the order they appear in $\operatorname{Tr}(\alpha, \beta)$.

Definition 1.2.3. Given $\alpha \leq \beta$ countable ordinals, the maximal weight $\varrho_{1}(\alpha, \beta)$ is defined as

$$
\varrho_{1}(\alpha, \beta)=\max \varrho_{0}(\alpha, \beta)
$$

It shall also be denoted by $e_{\beta}(\alpha)=\varrho_{1}(\alpha, \beta)$.

This is the most important of the weight functions on this section. Let's define two more functions. They are quite simple, but Todorcevic showed on [Tod07] that they can also lead to coherent sequences of functions, something we will explain later in this section. They also appear on [Tod10], another one of Todorcevic's works.

Definition 1.2.4. Given $\alpha<\beta$ countable ordinals, we define the length function by

$$
\varrho_{2}(\alpha, \beta)=|\operatorname{Tr}(\alpha, \beta)|-1
$$

and the last step function by 


$$
\varrho_{3}(\alpha, \beta)=\left\{\begin{array}{l}
1 \Longleftrightarrow\left|C_{\beta_{\varrho_{2}(\alpha, \beta)-1}} \cap \alpha\right|=\varrho_{1}(\alpha, \beta) \\
0 \Longleftrightarrow\left|C_{\beta_{\varrho_{2}(\alpha, \beta)-1}} \cap \alpha\right| \neq \varrho_{1}(\alpha, \beta)
\end{array}\right.
$$

So, the length function is literally the number of steps it takes to walk from $\beta$ to $\alpha$ in $\operatorname{Tr}(\alpha, \beta)$ while the last step function checks if the last step before $\alpha$ had the maximum weight.

Let's go back to the maximal weight now. We also denote the maximal weight by $e_{\beta}(\alpha)=\varrho_{1}(\alpha, \beta)$ because this way we can define a function $e_{\beta}: \beta \longrightarrow \omega$ for every $\beta<\omega_{1}$. With that in hand, we can define the sequence $\left\langle e_{\beta}: \beta<\omega_{1}\right\rangle$ of functions with increasing domains. This way it makes sense for us to ask if they are coherent, which means...

Definition 1.2.5. Given a sequence $\left\langle f_{\beta}: \beta<\omega_{1}\right\rangle$ of functions such that $f_{\beta}: \beta \longrightarrow \omega_{1}$, we say that the sequence is coherent if for all $\beta_{0}<\beta$, the restriction $f_{\beta} \uparrow_{\beta_{0}}$ is different than $f_{\beta_{0}}$ in only finite many points.

Before answering this question, we will prove an interesting proposition that will help us see the maximal weight function with more clarity. Most importantly, it is also an important lemma for answering our question.

Lemma 1.2.6. Given $\alpha<\beta$ countable ordinals and $n<\omega$, there is $\alpha_{0}<\alpha$ such that for every $\alpha_{0}<\gamma<\alpha$, we have:

(i) $\operatorname{Tr}(\gamma, \beta)=\operatorname{Tr}(\gamma, \alpha) \cup \operatorname{Tr}(\alpha, \beta)$;

(ii) $\left|C_{\alpha} \cap \gamma\right|>n$.

Proof. By 1.1.3, we know that first item is true for every $\alpha_{0} \geq \max \mathrm{L}(\alpha, \beta)$. For the second item, if $\alpha$ is not limit, we set $\alpha_{0}$ to be the ordinal such that $\alpha=\alpha_{0}+1$, since $C_{\alpha}=\{\alpha\}$ in this case. If $\alpha$ is limit, then $C_{\alpha}$ is a crescent sequence converging to $\alpha$, meaning that as $\alpha_{0}$ increases, so does $\left|C_{\alpha} \cap \alpha_{0}\right|$. We just set $\alpha_{0}$ big enough to get the job done.

Corollary 1.2.7. Let $\beta$ be a given countable ordinal. Then, $e_{\beta}$ is finite-to-one and for every limit ordinal $\alpha<\beta$, we have

$$
\lim _{\xi \rightarrow \alpha} e_{\beta}(\xi)=\infty .
$$


Proof. Let's suppose there is $n<\omega$ such that $e_{\beta}^{-1}(n)$ is infinite. Then we can take a crescent sequence $\left\langle\gamma_{i}: i<\omega\right\rangle$ converging to some $\gamma \leq \beta$. With the proposition we've just proven, we can find a $\gamma^{\prime}<\gamma$ such that for every $\gamma^{\prime}<\alpha<\gamma$ we have $\left|C_{\gamma} \cap \alpha\right|>n$ and $\gamma \in \operatorname{Tr}(\alpha, \beta)$, which means that for every $i<\omega$ such that $\gamma_{i}>\gamma^{\prime}$, we have $e_{\beta}\left(\gamma_{i}\right)>n$, contradicting that $\gamma_{i}$ is in $e_{\beta}^{-1}(n)$.

The last part is basically a consequence of the first. Given a certain $M<\omega$, we have that

$$
e_{\beta}^{-1}(\{0,1,2, \cdots, M\})=e_{\beta}^{-1}(0) \cup e_{\beta}^{-1}(1) \cup \cdots \cup e_{\beta}^{-1}(M),
$$

which is a finite union of finite sets, meaning that $e_{\beta}^{-1}(\{0,1,2, \cdots, M\})$ is finite and so we can take an ordinal $\delta>\max e_{\beta}^{-1}(\{0,1,2, \cdots, M\})$ and thus we'll have that $e_{\beta}(\alpha)>M$ for all $\alpha>\delta$.

Hopefully, this will give us some insight on how the maximal weight behaves. Notice that even though $e_{\beta}$ goes to infinity at every limit point less than $\beta$, it is also growing to infinity as it approaches $\beta$. If $\beta$ itself is a limit ordinal, then this result proves it. If it's not, then there is some limit ordinal $\alpha<\beta$ and some $k<\omega$ such that $\beta=\alpha+k$, and so the result yields for $\alpha$ and the rest of the functions are only finite many points.

Also, the functions $e_{\beta}$ being finite-to-one is an important fact we will recall through this document a few times.

We are now prepared to answer the question we left open. Let's get to it.

Proposition 1.2.8. The sequence $\left\langle e_{\beta}: \beta<\omega_{1}\right\rangle$ of functions is coherent.

Proof. Given $\beta<\beta^{\prime}$ countable ordinals, we will suppose that there are infinite many points where $e_{\beta}$ is different than $e_{\beta^{\prime}}$. This means that we can take a sequence $\left\langle\alpha_{k}: k<\omega\right\rangle$ of such points that is crescently converging to some $\delta \leq \beta$. We can use lemma 1.2.6 to find a $\delta_{0}<\delta$ such that for every $\delta_{0}<\alpha<\delta$, we have

1. $\operatorname{Tr}(\alpha, \beta)=\operatorname{Tr}(\alpha, \delta) \cup \operatorname{Tr}(\delta, \beta)$;

2. $\operatorname{Tr}\left(\alpha, \beta^{\prime}\right)=\operatorname{Tr}(\alpha, \delta) \cup \operatorname{Tr}\left(\delta, \beta^{\prime}\right)$;

Since $\alpha_{k} \rightarrow \delta$, there is $m<\omega$ such that $\delta_{0}<\alpha_{k}<\delta$ for all $k>m$. Notice how 1 and 2 mean that $\delta$ is in both $\operatorname{Tr}\left(\alpha_{k}, \beta\right)$ and $\operatorname{Tr}\left(\alpha_{k}, \beta^{\prime}\right)$, so since $e_{\beta}\left(\alpha_{k}\right) \neq e_{\beta^{\prime}}\left(\alpha_{k}\right)$, we get that 1 
and 2 are implying that the difference is coming from $\operatorname{Tr}(\delta, \beta)$ and $\operatorname{Tr}\left(\delta, \beta^{\prime}\right)$. We have by the definition of the maximal weight that

$$
\begin{aligned}
\operatorname{Tr}(\delta, \beta)=\left\{\beta_{0}, \cdots, \beta_{l}\right\} & , \quad \operatorname{Tr}\left(\delta, \beta^{\prime}\right)=\left\{\beta_{0}^{\prime}, \cdots, \beta_{l^{\prime}}^{\prime}\right\} \\
e_{\beta}(\delta)=\max \left\{\left|C_{\beta_{0}} \cap \delta\right|, \cdots,\left|C_{\beta_{l-1}} \cap \delta\right|\right\} & , \quad e_{\beta^{\prime}}(\delta)=\max \left\{\left|C_{\beta_{0}^{\prime}} \cap \delta\right|, \cdots,\left|C_{\beta_{l^{\prime}-1}^{\prime}} \cap \delta\right|\right\}
\end{aligned}
$$

and so we can take $\gamma=\max \left\{\left[\left(C_{\beta_{0}} \cap \delta\right) \cup \cdots \cup\left(C_{\beta_{l-1}} \cap \delta\right)\right] \cup\left[\left(C_{\beta_{0}^{\prime}} \cap \delta\right) \cup \cdots \cup\left(C_{\beta_{l^{\prime}-1}^{\prime}} \cap \delta\right)\right]\right\}$. Notice that $\gamma<\delta$, so we can also take $m<\omega$ such that, for all $k>m, \gamma<\alpha_{k}$ while 1 and 2 also holds. This way, $C_{\beta_{i}} \cap \delta=C_{\beta_{i}} \cap \alpha_{k}$ and $C_{\beta_{j}^{\prime}} \cap \delta=C_{\beta_{j}^{\prime}} \cap \alpha_{k}$ for all $k$, $i$ and $j$, since we made sure that there is nothing in $C_{\beta_{i}}$ and $C_{\beta_{j}^{\prime}}$ between $\delta$ and $\alpha_{k}$. This means that $e_{\beta}\left(\alpha_{k}\right)=e_{\beta}(\delta)$ and $e_{\beta^{\prime}}\left(\alpha_{k}\right)=e_{\beta^{\prime}}(\delta)$ for all $k>m$, which means that there are infinite many ordinals with image in $e_{\beta}(\delta)$ and $e_{\beta^{\prime}}(\delta)$, contradicting the fact that both $e_{\beta}$ and $e_{\beta^{\prime}}$ are finite-to-one.

And there we have it. The results from 1.1.3, 1.1.4, 1.2.7 and 1.2.8 are very important in the construction of the L-space. We will recall them a lot for this matter. To end this section, we will define one last function.

Definition 1.2.9. Given $\alpha<\beta$ countable ordinals, we define $\Delta(\alpha, \beta)$ to be the following functions:

$$
\begin{gathered}
\Delta(\alpha, \beta)=\min \left\{\xi<\alpha: e_{\alpha}(\xi) \neq e_{\beta}(\xi)\right\} \cup\{\alpha\} ; \\
\Delta_{0}(\alpha, \beta)=\min \left\{\xi<\alpha: \varrho_{0}(\xi, \alpha) \neq \varrho_{0}(\xi, \beta)\right\} \cup\{\alpha\} .
\end{gathered}
$$

\subsection{Full lower trace}

This will be the last concept to introduce about minimal walks. This last idea is a generalization of the lower trace, as the name of the section suggests.

Definition 1.3.1. Given $\alpha<\beta$ countable ordinals, the full lower trace of the minimal walk from $\beta$ to $\alpha$ is the set $\mathrm{F}(\alpha, \beta)$ recursively constructed by:

- $\mathrm{F}(\alpha, \alpha)=\{\alpha\}$

- $\mathrm{F}(\alpha, \beta)=\mathrm{F}\left(\alpha, \min \left(C_{\beta} \backslash \alpha\right)\right) \cup \bigcup_{\xi \in C_{\beta} \cap \alpha} \mathrm{F}(\xi, \alpha)$. 
This one is harder to give an alternate definition to. It's easy to see that $\mathrm{L}(\alpha, \beta) \subset$ $\mathrm{F}(\alpha, \beta) \leq \alpha$, as it keeps recalling all other ordinals that were refered to on the walk from $\beta$ to $\alpha$, which makes it larger but still finite.

Both the full code and the full lower trace are important ideas to find Suslin trees and categorize Mahlo cardinals, as done in [Tod07]. The full lower trace and the full code are linked and have two analog properties that we will now prove.

Proposition 1.3.2. Given $\alpha \leq \beta \leq \gamma$ countable ordinals, we have that:

(i) $\mathrm{F}(\alpha, \gamma) \subset \mathrm{F}(\alpha, \beta) \cup \mathrm{F}(\beta, \gamma)$;

(ii) $\mathrm{F}(\alpha, \beta) \subset \mathrm{F}(\alpha, \gamma) \cup \mathrm{F}(\beta, \gamma)$.

Proof. First, note how if there is any equality on $\alpha \leq \beta \leq \gamma$, these inclusions are obvious. So we just have to prove the case $\alpha<\beta<\gamma$, and assuming that makes an induction on $\gamma$ much easier. And that's what we are going to do.

Let $\gamma_{1}=\min \left(C_{\gamma} \backslash \alpha\right)$. Now first, let's do item (i). By the definition of the full lower trace, we have to prove that

$$
\mathrm{F}\left(\alpha, \gamma_{1}\right) \subset \mathrm{F}(\alpha, \beta) \cup \mathrm{F}(\beta, \gamma)
$$

and that for every $\xi \in C_{\gamma} \cap \alpha$,

$$
F(\xi, \alpha) \subset \mathrm{F}(\alpha, \beta) \cup \mathrm{F}(\beta, \gamma)
$$

Let's start by looking into $\mathrm{F}\left(\alpha, \gamma_{1}\right)$. By induction hypothesis, we get two cases: $\gamma_{1}<\beta$ or $\gamma_{1} \geq \beta$. On the first case, we apply item (ii) to $\gamma_{1}$ and get

$$
\mathrm{F}\left(\alpha, \gamma_{1}\right) \subset \mathrm{F}(\alpha, \beta) \cup \mathrm{F}\left(\gamma_{1}, \beta\right)
$$

Since $\alpha<\beta$, we have that $\gamma_{1}$ is also on $C_{\gamma} \cap \beta$, which means that by definition of $\mathrm{F}(\beta, \gamma)$,

$$
\mathrm{F}\left(\gamma_{1}, \beta\right) \subset \mathrm{F}(\beta, \gamma) \therefore \mathrm{F}\left(\alpha, \gamma_{1}\right) \subset \mathrm{F}(\alpha, \beta) \cup \mathrm{F}(\beta, \gamma)
$$

is just what we wanted. For the case $\gamma \geq \beta$, we apply item (i) to $\gamma_{1}$ and get

$$
\mathrm{F}\left(\alpha, \gamma_{1}\right) \subset \mathrm{F}(\alpha, \beta) \cup \mathrm{F}\left(\beta, \gamma_{1}\right)
$$


Since $\gamma_{1}=\min \left(C_{\gamma} \backslash \alpha\right) \geq \beta>\alpha$, this means that $C_{\gamma}$ has no points between $\alpha$ and $\beta$, so we also have that $\gamma_{1}=\min \left(C_{\gamma} \backslash \beta\right)$. With that in mind, we have by definition of $\mathrm{F}(\beta, \gamma)$ that

$$
\mathrm{F}\left(\beta, \gamma_{1}\right) \subset \mathrm{F}(\beta, \gamma) \therefore \mathrm{F}\left(\alpha, \gamma_{1}\right) \subset \mathrm{F}(\alpha, \beta) \cup \mathrm{F}(\beta, \gamma)
$$

which ends case $\mathrm{F}\left(\alpha, \gamma_{1}\right)$. Now let's delve into $\mathrm{F}(\xi, \alpha)$. By applying item (ii), we get

$$
\mathrm{F}(\xi, \alpha) \subset \mathrm{F}(\xi, \beta) \cup \mathrm{F}(\alpha, \beta)
$$

In a similar fashion to last case, $\alpha<\beta$ implies that $\xi$ is also in $C_{\gamma} \cap \beta$, which once again leads us to

$$
\mathrm{F}(\xi, \beta) \subset \mathrm{F}(\beta, \gamma) \therefore \mathrm{F}(\xi, \alpha) \subset \mathrm{F}(\alpha, \beta) \cup \mathrm{F}(\beta, \gamma)
$$

Proving item (ii) is basically the same. We use the same arguments again, so we will end this demonstration here.

This next proposition is very similar to the last one. In fact, it is so similar that it's demonstration is also pretty much the same as this one. So, to avoid doing the same things over and over, we will skip it's demonstration. For the readers who want more details, you can check this demonstration on [Tod07].

Proposition 1.3.3. Given $\alpha \leq \beta \leq \gamma$ countable ordinals, we have that

(i) $\varrho_{0}(\alpha, \beta)=\varrho_{0}(\min (F(\beta, \gamma) \backslash \alpha), \beta) \wedge \varrho_{0}(\alpha, \min (F(\beta, \gamma) \backslash \alpha))$;

(ii) $\varrho_{0}(\alpha, \gamma)=\varrho_{0}(\min (F(\beta, \gamma) \backslash \alpha), \gamma) \wedge \varrho_{0}(\alpha, \min (F(\beta, \gamma) \backslash \alpha))$.

And that should cover the basics. We need to cover the oscilation functions but to do so, we need to develop some other tools. These tools will also be very important to construct the L-space and to prove almost every other important theorem on this document, so we will dedicate this next chapter entirely to them. 


\section{Chapter 2}

\section{Countable Elementary Submodels}

Countable elementary submodels have been out for quite some years now. They are very powerful tools in set theory, used in a number of technical demonstrations. The theory and it's applications can be read on [Dow88], and also on chapter 24 of [Wee97]. We won't delve into logic and model theory too deep here on this document. Instead, we will only show and prove what we need and assume that the reader knows the basics to understand it. We will talk about Lowenheim-Skolem theorem since it is important for it.

So, the idea behind countable elementary submodels is that you can use the transitivity of formulas between the models to build very specific sets. Given a certain proof we are trying to do, we will always only use a finite number of axioms from ZFC, so we can find a model for these. Usually, $H(\theta)$ for a large enough cardinal $\theta$ does it. Thus, LowenheimSkolem theorem will find a countable elementary submodel containing all the sets and tools we are working with. For all our purposes, the model $H\left(\omega_{1}^{+}\right)$will cover all we need most of the time.

\subsection{Basics}

Let's start by recalling what it means to be an elementary submodel.

Definition 2.1.1. Given $M$ an $\mathcal{L}$-structure of some language $\mathcal{L}$, an elementary submodel of $M$ is an $\mathcal{L}$-structure $N \subset M$ such that for all $\mathcal{L}$-formula $\phi$ with parameters in $N, N \models \phi$ if and only if $M \models \phi$. We denote them by $N \prec M$.

This is huge. For example, it means that anything you can build in $N$, if $M$ under- 
stands each passage, then you can also build it in $M$ and it is often the same object due to absoluteness.

Example. For $\mathcal{L}=\{<\}$ and $\mathbb{R}$ being it's $\mathcal{L}$-structure, take $\mathbb{R}^{+}=(0,+\infty)$. Due to the logarithmic function being a strictly crescent bijection, we get that $\mathbb{R}^{+} \prec \mathbb{R}$. For example, $\mathbb{R}^{+}$satisfies properties such as $(\forall x)(\exists y)[x<y],(\forall x)(\exists y)[y<x]$ and $(\forall x)(\forall y)(\exists t)[x<t<y]$.

Notice that we mentioned a lot that we want our submodels to be countable, and $\mathbb{R}^{+}$is not countable. So it isn't the best example for the properties we are going to use. Intuitively, we could think that $\mathbb{Q} \prec \mathbb{R}$, but $\mathbb{Q}$ does not satisfies the property of the supremum.

The Lowenheim-Skolem theorem can find countable elementary submodels for most theories and models, at least for all our purposes. Let's recall it and some of it's consequences and implications.

Theorem 2.1.2 (Downward Lowenheim-Skolem's Theorem). Let $M$ be an infinite $\mathcal{L}$-structure of some language $\mathcal{L}$. For all cardinals $\kappa \leq|M|$ and $S \subset M$ such that $|S|=\kappa$, there is an elementary substructure $N \prec M$ such that $|N|=\kappa+|\mathcal{L}|$ and $S \subset N$.

There are many known proofs of this theorem around. We will try to take a simple route.

Proof. For each non-empty set $D \subset M$ definable, use the axiom of choice to take an element $e(D) \in D$. Given some set $X \subset M$, let's define

$$
c(X)=X \cup\{e(D): D \text { is definable over } X \text { and } D \neq \varnothing\}
$$

Since there are at most $|X|+|L|$ definable sets over $X$, then $|c(X)| \leq|X|+|L|$. Given $N_{0}=S$, let's recursively define

$$
N_{n+1}=c\left(N_{n}\right)
$$

Taking $N=\bigcup\left\{N_{n}: n<\omega\right\}$, we get that $|N|=\kappa+|L|$ and by the Tarski-Vaught test, $N \prec M$.

This theorem says exactly what we were looking for. We take $S$ to be the subset of everything we need and it means we have a countable elementary submodel with it, as long as our theory is a first-order logic and $S$ and $L$ are both countable, which in our case, they will always be. 
Theorem 2.1.3 (Upward Lowenheim-Skolem Theorem). Let $M$ be an infinite $\mathcal{L}$-structure of some language $\mathcal{L}$. For all cardinals $\kappa \geq|M|+|L|$, there is an extension $N \succ M$ such that $|N|=\kappa$.

Proof. Let's start by taking a set $\left\{c_{\alpha}: \alpha<\kappa\right\}$ of new constant symbols. Now, define the set

$$
T=\operatorname{eldiag}(M) \cup\left\{c_{\alpha}: \alpha<\kappa\right\} .
$$

Any finite subset of $T$ will be a finite amount of the new constants and the rest will be from eldiag $(M)$, and since $M$ is consistent, that means we can also find a model for this finite subset of $T$. This proves $T$ is consistent by compactness. If it's consistent, we can find $N \models T$, which means $M \succ N$ because $T$ contains eldiag $(M)$. Since we added $\kappa>|M|+|L|$ new constant symbols, $|N|=\kappa$, which ends our proof.

We won't really use the upward theorem, but since we are talking about the downward theorem, we might as well talk about the upward too, since it is equally important.

Let's talk about it's impact. First, it means that first-order logic theories that have infinite models can't be categorical, in other words, their models are not all isomorphic since we can find models of all cardinalities. In mathematics history, this was the first sign that mathematics couldn't have a "well behaved theory", that is, first-order categorical and consistent.

For some examples of it's intuition shattering consequences, it showed the existence of countable submodels of $H\left(\omega_{1}^{+}\right)$that recognize $\omega_{1}$ as uncountable. That seems contradictory, but that happens because for a set $A$ to be countable means existing some bijection $f$ : $\mathbb{N} \longrightarrow A$. If a countable model $M$ has his copy of $\omega_{1}$, even though his copy is countable in $H\left(\omega_{1}^{+}\right)$, it will not possess a bijection from $\mathbb{N}$ onto $\omega_{1}$. This means that some sets can be countable in some models and uncountable in others, and no rule is really being broken. It's just that our perception of cardinality as quantity is flawed in regards to cardinality's definition.

Other crazy things this theorem brought to light is the existence of uncountable models for true arithmetics. That seemed counterintuitive as inductive sets are supposed to be countable, but what is happening is, again, our perception being wrong in regards to the axioms of inductivity. At a glance, it would also mean that there were models of any size for $\mathcal{L}=(0,1,+, \times,<)$ with the theory of complete ordered fields, contradicting the fact that all of those fields are isomorphic to $\mathbb{R}$. That is not the case because completeness' axiomatization is not actually first-order logic. 
Well, with all of that, we can all agree that mathematics is not at all as intuitive as we think it is sometimes, and certainly will surprise us no matter how deep we think our knowledge of mathematics is. But we digress... Let's move on.

The next section will be where we explain and show the usefulness of countable elementary submodels. We will prove basic facts that everyone uses to work with them. Most are very easy and straightforward, but all are powerful nonetheless.

\subsection{Useful facts}

Proposition 2.2.1. Given $M$ a countable elementary submodel of $H(\theta)$ for any cardinal $\theta$ and some set $A \in M$, then $A$ is countable if and only if $A \subset M$.

Proof. If $A \subset M$, then $A$ is countable. If $A$ is countable, then by elementarity $M$ also thinks $A$ is countable, meaning there is $f: \mathbb{N} \longrightarrow A$ a bijective function. So $M$ contains it's range, finishing our proof.

Proposition 2.2.2. Given $M$ a countable elementary submodel of $H(\theta)$ for any cardinal $\theta$, then $\delta=M \cap \omega_{1}$ is an ordinal.

Proof. Since $\delta \subset \omega_{1}$, we already know that $\in$ is a well order. To see that it's transitive, just notice that $\delta$ only has countable elements, so they are all also subsets of $M$ by 2.2 .1 since $\delta \subset M$.

Proposition 2.2.3. Given $M$ a countable elementary submodel of $H(\theta)$ for any cardinal $\theta$ and some set $A \in M$, if $M \cap \omega_{1} \in A$, then $A$ is uncountable.

Proof. If $M \cap \omega_{1} \in A$, then $A \not \subset M$ and thus by 2.2 .1 it is uncountable.

Although this last proposition is simple, it is a reliable way to prove that elements in countable elementary submodels are uncountable. In fact, not only it needs to have $\delta$, it can have any other element that $M$ does not.

Proposition 2.2.4. Given $M$ a countable elementary submodel of $H(\theta)$ for any cardinal $\theta$ and some set $A \subset \omega_{1}$ in $M$, then $A$ is uncountable if and only if $A \cap M$ is unbounded in $M \cap \omega_{1}$. 
Proof. No uncountable set is bounded in $\omega_{1}$. Notice that since $M$ 's only ordinals are those less than $M \cap \omega_{1}=\delta$, if $A \cap M$ was bounded in $\delta$ by some countable ordinal $\gamma<\delta$, then $M \models A \cap \omega_{1}<\gamma$ and thus by elementarity, $H\left(\omega_{1}^{+}\right) \models A \cap \omega_{1}<\gamma$.

Proposition 2.2.5. Given a countable set $F \subset H(\theta)$ for any cardinal $\theta$, then the countable elementary submodels $M$ of $H(\theta)$ that contain $F$ are such that the ordinals $M \cap \omega_{1}$ form a set that has a club of $\omega_{1}$.

Proof. By Lowenheim-Skolem downwards theorem, we can find a countable elementary submodel $M$ containing $F \cup\{\alpha\}$ for each ordinal $\alpha<\omega_{1}$. By 2.2.2, there must be uncountably many different submodels like this, thus the ordinals $M \cap \omega_{1}$ form an uncountable set in $\omega_{1}$ and are then unbounded. To see that it contains a $c l u b$, we can see that it already contains all it's limit ordinals by seeing that every limit ordinal in it has a countable crescent sequence converging to it, so we can take the union of it's models and that will show that the limit is also included in the set.

Proposition 2.2.6. Given $M$ a countable elementary submodel of $H(\theta)$ for any cardinal $\theta$ and $X \in H\left(\omega_{1}^{+}\right)$definable by a formula with parameters in $M$, then $X \in M$.

Proof. By elementarity.

These are pretty much everything one needs to know to work with countable elementary submodels. We will use them to construct the L space, and will only need these results. We can move on to the next session now. 


\section{Chapter 3}

\section{The L Space}

\subsection{Oscillations of the lower trace}

We will begin by introducing another idea that also appears in both [Moo06] and [Tod07]. This is the base of Moore's construction. This idea is the foundation of many applications of minimal walks, since we basically use the results we will derive here to link minimal walks to other fields such as model theory and general topology.

Definition 3.1.1. Given $\alpha<\beta$ countable ordinals, we will write $\operatorname{Osc}_{\mathrm{L}}(\alpha, \beta)$ and $\operatorname{osc}_{\mathrm{L}}(\alpha, \beta)$ to denote the following:

- $\operatorname{Osc}_{\mathrm{L}}(\alpha, \beta)=\left\{\xi_{i+1} \in L(\alpha, \beta): e_{\alpha}\left(\xi_{i}\right) \leq e_{\beta}\left(\xi_{i}\right)\right.$ and $\left.e_{\alpha}\left(\xi_{i+1}\right)>e_{\beta}\left(\xi_{i+1}\right)\right\}$;

- $\operatorname{osc}_{\mathrm{L}}(\alpha, \beta)=\left|\operatorname{Osc}_{\mathrm{L}}(\alpha, \beta)\right|$.

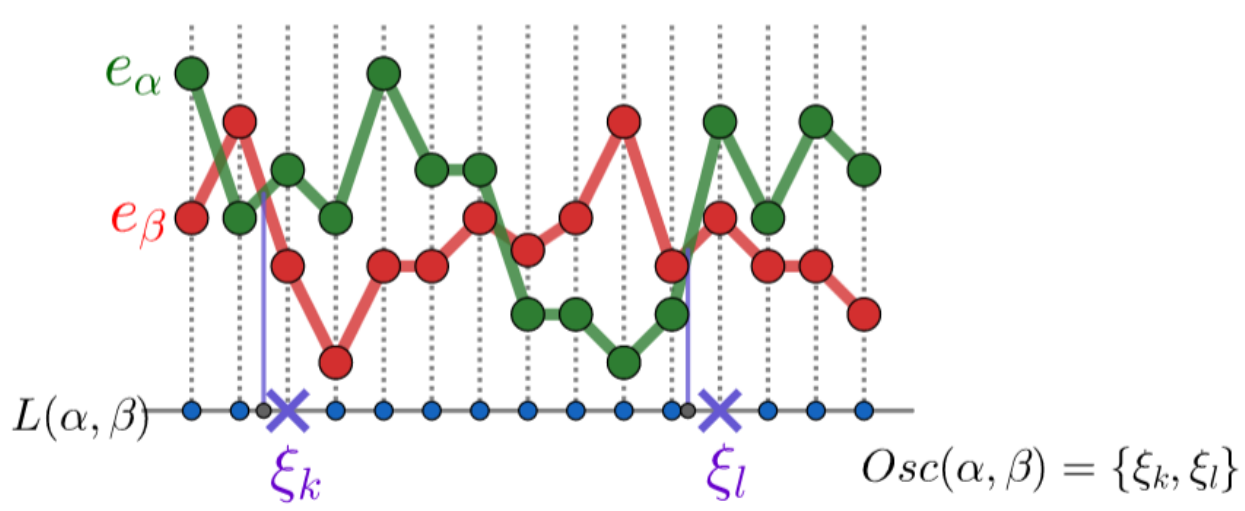

The oscillation of $\alpha$ and $\beta$ is basically the set of all points in $\xi$ where $e_{\alpha}$ surpasses $e_{\beta}$. 
We basically need one combinatorial theorem about the oscillation on the lower trace to build the $\mathrm{L}$ space. The use of those oscillations were first used in this very construction in 2006 by Moore in [Moo06].

This next part is a little complicated. We want to prove an important theorem that's a bit hard to prove. To do so, we will first prove a lemma that's also hard to prove. And to state. We will do it as Moore did in [Moo06].

Lemma 3.1.2. Let $\mathcal{A} \subset\left[\omega_{1}\right]^{k}$ and $\mathcal{B} \subset\left[\omega_{1}\right]^{l}$ be uncountable with pairwise disjoint elements. Then, there exists a club in $\omega_{1}$ with ordinals $\delta<\omega_{1}$ such that if $a \in \mathcal{A} \backslash \delta, b \in \mathcal{B} \backslash \delta$ and $R \in\{=,>\}$, then there are $a^{+} \in \mathcal{A} \backslash \delta$ and $b^{+} \in \mathcal{B} \backslash \delta$ such that for all $i<k$ and $j<l$,

(i) $\max \mathrm{L}(\delta, b(j))<\Delta\left(a(i), a^{+}(i)\right)$ and $\max \mathrm{L}(\delta, b(j))<\Delta\left(b(j), b^{+}(j)\right)$;

(ii) $\mathrm{L}(\delta, b(j))$ is an initial segment of $\mathrm{L}\left(\delta, b^{+}(j)\right)$;

(iii) If $\xi$ is in $L^{+}=\mathrm{L}\left(\delta, b^{+}(j)\right) \backslash \mathrm{L}(\delta, b(j))$, then $e_{a^{+}(i)}(\xi) R e_{b^{+}(j)}(\xi)$.

Before we prove this lemma, let's first understand what it's saying. Given $\delta, a$ and $b$, we can find $a^{+}$and $b^{+}$such that $\mathrm{L}\left(\delta, b^{+}(j)\right)=\mathrm{L}(\delta, b(j)) \cup L^{+}$and their maximal weight functions work as shown there on the scheme.

$$
\begin{gathered}
\xi \in L(\delta, b(j)) \Longrightarrow \begin{array}{l}
e_{a(i)}(\xi)=e_{a^{+}(i)}(\xi) \\
e_{b(j)}(\xi)=e_{b^{+}(j)}(\xi)
\end{array} \\
L(\delta, b(j)) \\
L\left(\delta, b^{+}(j)\right)=L(\delta, b(j)) \cup L^{+}
\end{gathered}
$$

Also, when we say $\mathcal{A} \backslash \delta$ we mean all sets in $\mathcal{A}$ whose elements are all greater than $\delta$, and when we write $a(i)$ we mean the i-th element of $a$, taken on the natural order of the ordinals. This proof will be a good showcase of the power of countable elementary submodels. The idea of the proof is to take a countable elementary submodel of $H\left(\omega_{1}^{+}\right)$and see that these properties we stated are all understandable by it and thus, we can use the properties of the second chapter to great effect. We are ready to start the proof.

Proof. Let $M$ be any countable elementary submodel of $H\left(\omega_{1}^{+}\right)$with everything that's important and let $\delta=M \cap \omega_{1}$. By 2.2.5, we only need to prove that these $\delta$ are the ones we are looking for, as by the proposition's statement. 
We are going to do this proof divided in the two cases of $R$ either being $=$ or $>$. First, let's start with the case $R$ being $=$.

Since the lower trace is finite and $\mathrm{L}(\delta, b(j))<\delta$, we can take an ordinal $\gamma_{0}<\delta$ greater than $\mathrm{L}(\delta, b(j))$ for all $j<l$ and, and by 1.2.8, we can also make it such that $e_{a(i)}(\xi)=e_{b(j)}(\xi)$ for all $i<k, j<l$ and $\gamma_{0}<\xi<\delta$. Also by 1.1.4, we can pick a $\gamma<\delta$ such that for all $\gamma<\xi<\delta, \gamma_{0}<\mathrm{L}(\xi, \delta)$.

Now, consider the following set:

$D=\left\{\delta^{+} \in \omega_{1}\right.$ : there are $a^{+} \in \mathcal{A} \backslash \delta^{+}$and $b^{+} \in \mathcal{B} \backslash \delta^{+}$satisfying properties $1,2,3$ and 4$\}$

where properties 1, 2, 3 and 4 are as follows:

1. For all $i<k$ and $j<l, e_{a^{+}(i)} \uparrow_{\gamma_{0}}=e_{a(i)} \uparrow_{\gamma_{0}}$ and $e_{b^{+}(j)} \uparrow_{\gamma_{0}}=e_{b(j)} \uparrow_{\gamma_{0}}$;

2. For all $j<l, \mathrm{~L}\left(\delta^{+}, b^{+}(j)\right)=\mathrm{L}(\delta, b(j))$;

3. For all $\gamma<\xi<\delta^{+}, \gamma_{0}<\mathrm{L}\left(\xi, \delta^{+}\right)$;

4. For all $\gamma_{0}<\xi<\delta^{+}, i<k$ and $j<l, e_{a^{+}(i)}(\xi)=e_{b^{+}(j)}(\xi)$.

Notice how $D$ is definable in $M$, since all constant parameters used in it's properties are $\gamma, \gamma_{0}, e_{a(i)} \Upsilon_{\gamma_{0}}$ and $e_{b(j)} \uparrow_{\gamma_{0}}$, which are in $M$ due to the restriction to $\gamma_{0}$, which is less than $\delta$, and due to 1.2 .8 making them different from $e_{\gamma_{0}}$ in only finite many points, thus we can define them manually in $M$ for all $i<k$ and $j<l$. Thus, we conclude that $D \in M$ due to 2.2.6.

Furthermore, you can clearly see that $\delta$ satisfies all these conditions by taking $a^{+}=a$ and $b^{+}=b$, which by 2.2.3 means that $D$ is in fact uncountable. This means that there is some $\delta^{+}>\delta$ in $D$, since uncountability is also unboundedness. So, let's take $a^{+} \in \mathcal{A} \backslash \delta^{+}$ and $b^{+} \in \mathcal{B} \backslash \delta^{+}$witnessing that $\delta^{+} \in D$. See that 1 implies that $\gamma_{0} \leq \Delta\left(a(i), a^{+}(i)\right)$ and $\gamma_{0} \leq \Delta\left(b(j), b^{+}(j)\right)$ for all $i<k$ and $j<l$, and since we took $\gamma_{0}$ such that $\mathrm{L}(\delta, b(j))<\gamma_{0}$ for all $j<l$, we have (i) being held.

Now, take $L^{+}=\mathrm{L}\left(\delta, \delta^{+}\right)$. By $2, \mathrm{~L}(\delta, b(j))=\mathrm{L}\left(\delta^{+}, b^{+}(j)\right)$. Since $\mathrm{L}(\delta, b(j))<\gamma_{0}$, with 3 we can conclude that $\mathrm{L}(\delta, b(j))=\mathrm{L}\left(\delta^{+}, b^{+}(j)\right)<L^{+}$. Thus, with 1.1.3, we conclude that

$$
\mathrm{L}\left(\delta, b^{+}(j)\right)=\mathrm{L}\left(\delta^{+}, b^{+}(j)\right) \cup L^{+}=\mathrm{L}(\delta, b(j)) \cup L^{+},
$$

which is (ii) as we wanted. 
For (iii), note that it implies from 4 , since $\gamma_{0}<L^{+}<\delta^{+}$and $R$ is $=$. This concludes the first part of this demonstration. Now we are going to prove the case where $R$ is $>$. Let us define a set $E$ of all limit ordinals $\nu<\omega_{1}$ such that for all $a_{0} \in \mathcal{A} \backslash \nu$, all $\nu_{0}<\nu, \epsilon<\omega_{1}$, $n<\omega$ and all finite sets $L^{+} \subset \omega_{1} \backslash \nu$, there is an $a_{1} \in \mathcal{A} \backslash \epsilon$ with $\nu_{0} \leq \Delta\left(a_{0}(i), a_{1}(i)\right)$ and $e_{a_{1}(i)}(\xi)>n$ whenever $i<k$ and $\xi \in L^{+}$. We can see by 2.2.6 that $E$ is in $M$, since, just like $D$, it understands both $\nu_{0} \leq \Delta\left(a_{0}(i), a_{1}(i)\right)$ and $e_{a_{1}(i)}(\xi)>n$, even though it does not understands the rest, it doesn't matter. The first thing we are going to prove is that $E$ is in fact uncountable. We will do this by proving that $\delta \in E$, just like we did with $D$.

So, let's take $a_{0}, \nu_{0}, \epsilon, n$ and $L^{+}$as in the definition of $E$ for $\nu=\delta$. If we can find an $a_{1}$ for a large $\nu_{0}$, it will also serve for all smaller $\nu_{0}$ due to 1.2.7. So all we need to do is find an $a_{1}$ for a large enough $\nu_{0}$, which in this case, assuming $\nu_{0}$ is an upper bound for all $\xi<\delta$ such that $e_{a_{0}(i)}(\xi) \leq n$ for some $i<k$. Now, let's take a set $\mathcal{A}_{0}=\left\{a \in \mathcal{A} \backslash \nu_{0}: e_{a(i)} \uparrow_{\nu_{0}}=\right.$ $e_{a_{0}(i)}\left\lceil_{\nu_{0}}\right.$ for all $\left.i<k\right\}$. You can see that because of the restriction, $\mathcal{A}_{0}$ is definable in $M$ and so it is in $M$ by 2.2.6. However, $a_{0} \in \mathcal{A}_{0}$, and since $a_{0}>\delta$ and is finite, it can't be in $M$. This means that $\mathcal{A}_{0}$ is uncountable by 2.2.1. Now we want to find $\delta^{+}$above $\epsilon, \delta$ and $\max L^{+}$and some $a_{1} \in \mathcal{A}_{0} \backslash \delta^{+}$such that for all $\xi \in L^{+}$and $i<k$, it holds that $e_{a_{1}(i)}(\xi)>n$. So, since $\delta^{+}$ has also to be less than $\omega_{1}$, this means that there actually countable many possibilities for elements in $\mathcal{A}_{0}$ that fails one of these conditions for any given $\delta^{+}$. Since $\mathcal{A}_{0}$ is uncountable, there must be some $\delta^{+}$and $a_{1}$ that fits all these conditions. So we just proved that $E$ is uncountable.

By 2.2.4, we can now find $\gamma_{0} \in E$ such that $\mathrm{L}(\delta, b(j))<\gamma_{0}<\delta$ for all $j<l$. Applying 1.1.4, find $\gamma<\delta$ such that for all $\gamma<\xi<\delta, \gamma_{0}<\mathrm{L}(\xi, \delta)$. Now we want to find a limit $\delta^{+}>\delta$ and a $b^{+} \in \mathcal{B} \backslash \delta^{+}$such that

1. For all $j<l, e_{b^{+}(j)} \Upsilon_{\gamma_{0}}=e_{b(j)} \uparrow_{\gamma_{0}}$

2. For all $j<l, \mathrm{~L}\left(\delta^{+}, b^{+}(j)\right)=\mathrm{L}(\delta, b(j))$;

3. For all $\gamma<\xi<\delta^{+}, \gamma_{0}<\mathrm{L}\left(\xi, \delta^{+}\right)$,

and we will find them just like we found the elements we needed to prove that $\delta \in E$. In fact, item 1 is just like the first thing we needed to do in that proof, so we know that there are actually uncountably many $b$ 's that satisfy it. Let's call $\mathcal{B}_{0}=\left\{b^{+} \in \mathcal{B} \backslash \gamma_{0}: e_{b^{+}(j)} \Upsilon_{\gamma_{0}}=e_{b(j)} \Upsilon_{\gamma_{0}}\right.$ for all $j<l\}$ the set of such $b$ 's, which we already know is uncountable. For item 2, consider $F=\left\{\delta^{+}<\omega_{1}\right.$ : there is $b^{+} \in \mathcal{B}_{0} \backslash \delta^{+}$such that $\mathrm{L}\left(\delta^{+}, b^{+}(j)\right)=\mathrm{L}(\delta, b(j))$ for all $\left.j<l\right\}$. It is definable in $M$ and $\delta$ is in it, so $F$ is uncountable. Now for item 3, take $F^{\prime}=\left\{\delta^{+} \in F\right.$ : $\left.\gamma<\xi<\delta^{+} \Longrightarrow \gamma_{0}<\mathrm{L}\left(\xi, \delta^{+}\right)\right\}$. Again, $F^{\prime} \in M$ and $\delta \in F^{\prime}$. This means that there are uncountably many $b^{+}$and $\delta^{+}$how we wanted. 
To end this demonstration, put $L^{+}=\mathrm{L}\left(\delta, \delta^{+}\right)$. By the definition of $E$, we can find $a^{+} \in \mathcal{A} \backslash \delta$ such that for all $i<k, j<l$ and $\xi \in L^{+}, \mathrm{L}(\delta, b(j))<\Delta\left(a(i), a^{+}(i)\right)$ and $e_{a^{+}(i)}(\xi)>e_{b^{+}(j)}(\xi)$. Now we are on a same point we were on the case $R$ is $=$ and we can end the demonstration just like we did on that case.

We are ready to state and prove the main theorem we talked about earlier.

Theorem 3.1.3. For every $\mathcal{A} \subset\left[\omega_{1}\right]^{k}$ and $\mathcal{B} \subset\left[\omega_{1}\right]^{l}$ uncountable sets with pairwise disjoint elements and $n<\omega$, there are $a \in \mathcal{A}$ and $b_{m} \in \mathcal{B}$ for every $m<n$ such that for all $i<k$, $j<l$ and $m<n$,

$$
\begin{gathered}
a<b_{m} \\
\operatorname{osc}_{\mathrm{L}}\left(a(i), b_{m}(j)\right)=\operatorname{osc}_{\mathrm{L}}\left(a(i), b_{0}(j)\right)+m .
\end{gathered}
$$

Before we start the proof, let's give an idea on how we are going to do it. Remember 3.1.2. It stated that, given $a$ and $b$, we could find $a^{+}$and $b^{+}$such that their weight functions are equal to $a$ and $b$ functions half the way and greater on the rest of the way. This means that we are creating an oscillation.

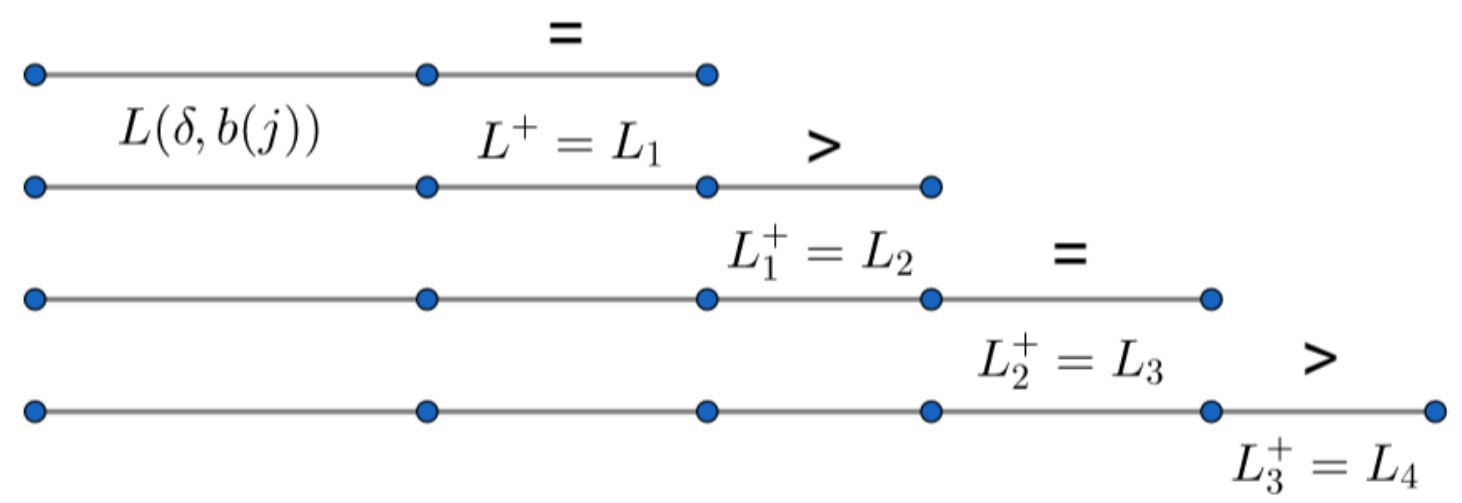

You see, we are going to again and again, each time on the points we got from the previous application, building upon the last interval, getting a bigger one where the maximal weight functions oscillate as many times as we want them to.

Proof. Let's fix some $\mathcal{A}$ and $\mathcal{B}$ as per the theorem's statement. We'll work with $n$ later. Now, let's take $M$ a countable elementary submodel of $H\left(\omega_{1}^{+}\right)$containing everything we need, setting $\delta=M \cap \omega_{1}$. With $\mathcal{A} \in M$ and $\mathcal{B} \in M$, by 3.1.2 we get that the whole club is in $M$, and as such, $\delta$ is in this $c l u b$ due to the demonstration of 3.1.2, where we proved that every countable elementary submodel $M$ containing these things we are using are such that $\delta=M \cap \omega_{1}$ is in the $c l u b$. 
Now, using 3.1.2, we will construct sequences $\left\langle a_{m}: m<\omega\right\rangle$ in $\mathcal{A} \backslash \delta,\left\langle b_{m}: m<\omega\right\rangle$ in $\mathcal{B} \backslash \delta$ and $\left\langle\xi_{m}: m<\omega\right\rangle$ in $\delta$ such that they have the following properties:

1. $\mathrm{L}\left(\delta, b_{m}(j)\right)$ is an initial segment of $\mathrm{L}\left(\delta, b_{m+1}(j)\right)$;

2. $\mathrm{L}\left(\delta, b_{m+1}(j)\right) \backslash \mathrm{L}\left(\delta, b_{m}(j)\right)=\mathrm{L}\left(\delta, b_{m+1}\left(j^{\prime}\right)\right) \backslash \mathrm{L}\left(\delta, b_{m}\left(j^{\prime}\right)\right)$ for all $j^{\prime}<l$;

3. $\xi_{m} \in \mathrm{L}\left(\delta, b_{m+1}(j)\right) \backslash \mathrm{L}\left(\delta, b_{m}(j)\right)$;

4. $\operatorname{Osc}\left(a_{m+1}(i), b_{m+1}(j) ; \mathrm{L}\left(\delta, b_{m+1}(j)\right)\right)=\operatorname{Osc}\left(a_{m}(i), b_{m}(j) ; \mathrm{L}\left(\delta, b_{m}(j)\right)\right) \cup\left\{\xi_{m}\right\}$;

5. If $m^{\prime}<m$, then $\xi_{m^{\prime}}<\Delta\left(a_{m}(i), a_{m+1}(i)\right)$ and $\xi_{m^{\prime}}<\Delta\left(b_{m}(j), b_{m+1}(j)\right)$;

6. $e_{a_{m}(i)}\left(\max \mathrm{L}\left(\delta, b_{m}(j)\right)\right)>e_{b_{m}(j)}\left(\max \mathrm{L}\left(\delta, b_{m}(j)\right)\right)$.

We will build them recursively. First, we will pick $a_{0} \in \mathcal{A} \backslash \delta$ and $b_{0} \in \mathcal{B} \backslash \delta$ arbitrarily. Now, suppose we have $a_{k+1}, b_{k+1}$ and $\xi_{k}$ for all $k<n$ and we are now going to define $a_{n+1}, b_{n+1}$ and $\xi_{n}$. First, we apply 3.1.2 with $R$ being $=$ to get $a_{n}^{+} \in \mathcal{A} \backslash \delta$ and $b_{n}^{+} \in \mathcal{B} \backslash \delta$. Next, we apply 3.1 .2 with $R$ being $>$ on $a_{n}^{+}$and $b_{n}^{+}$to get what we will call $a_{n+1} \in \mathcal{A} \backslash \delta$ and $b_{n+1} \in \mathcal{A} \backslash \delta$. Due to (ii) in 3.1 .2 , this means that

$$
\mathrm{L}\left(\delta, b_{n+1}(j)\right)=\mathrm{L}\left(\delta, b_{n}^{+}(j)\right) \cup L^{++}=\mathrm{L}\left(\delta, b_{n}(j)\right) \cup L^{+} \cup L^{++}
$$

and so 1 holds true. To see that 2 also holds true, let's look back at the demonstration of 3.1.2, where we find out who is $L^{+}$and who is $L^{++}$. We can see that it does not depends on $j$, corroborating our point. Now, to see 3 and 4 we need first to find out who is $\xi_{n}$. Looking at 4 , we know who he needs to be, and it would need to be in either $L^{+}$or $L^{++}$. Luckily, by (iii) in 3.1.2, we get

$$
\begin{gathered}
\xi \in L^{+} \Longrightarrow e_{a_{n}^{+}(i)}(\xi)=e_{b_{n}^{+}(j)}(\xi) \\
\xi \in L^{++} \Longrightarrow e_{a_{n+1}(i)}(\xi)>e_{b_{n+1}(j)}(\xi)
\end{gathered}
$$

Though because of (i), we get that $e_{a_{n}^{+}(i)} \uparrow_{L^{+}}=e_{a_{n+1}(i)} \Upsilon_{L^{+}}$and $e_{b_{n}^{+}(j)} \uparrow_{L^{+}}=e_{b_{n+1}(j)} \uparrow_{L^{+}}$, which gets us

$$
\begin{gathered}
\xi \in L^{+} \Longrightarrow e_{a_{n+1}(i)}(\xi)=e_{b_{n+1}(j)}(\xi) \\
\xi \in L^{++} \Longrightarrow e_{a_{n+1}(i)}(\xi)>e_{b_{n+1}(j)}(\xi)
\end{gathered}
$$

that means 4 and 3 holds true due to the definition of oscillations, which nets us $\xi_{n}=$ $\min L^{++}$. Notice that this choice of $\xi_{n}$ will also make it so 5 is true due to the arguments we used to make 4 also true. And to end this construction, notice that 6 is true due to $e_{a_{n+1}(i)}(\xi)>e_{b_{n+1}(j)}(\xi)$ being true for all $\xi \in L^{++}$and the fact that $\max \mathrm{L}\left(\delta, b_{n+1}(j)\right) \in L^{++}$ 
By now, it should be very clear. The whole lemma 3.1.2 is pieced together to create this sequence, which fits perfectly with what we tried to explain at the beginning before the proof.

We have to find $a$ such that $\operatorname{osc}_{\mathrm{L}}\left(a(i), b_{m}(j)\right)=\operatorname{osc}_{\mathrm{L}}\left(a(i), b_{0}(j)\right)+m$ and the sequence we build works on the oscillation on the set $\mathrm{L}\left(\delta, b_{m}(j)\right)$ instead of $\mathrm{L}\left(a(i), b_{m}(j)\right)$. First, let's fix that $n<\omega$ as per the theorem's statement. Now we can tackle this last issue.

Due to $n$ being fixed, we have finite many $\mathrm{L}\left(\delta, b_{m}(j)\right)$ for all $j<l$ and $m<n$, so we can take $\gamma_{0}<\delta$ an upper bound of all these sets. Also, with 1.2.6, we can also make this $\gamma_{0}$ an upper bound of all $\xi<\delta$ such that $e_{b_{m}(j)}(\xi) \neq e_{b_{m^{\prime}}\left(j^{\prime}\right)}(\xi)$ for all different $m<n, m^{\prime}<n$, $j<l$ and $j^{\prime}<l$.

Now with 1.1.4 and M's elementarity, we can select $a \in \mathcal{A}$ in a way such that $\gamma_{0}<$ $\mathrm{L}(a(i), \delta)$ and $\mathrm{L}\left(\delta, b_{n}(j)\right)<\Delta\left(a(i), a_{n}(i)\right)$. This way, it follows from 1.1.3 that, for every $m<n$,

$$
\mathrm{L}\left(a(i), b_{m}(j)\right)=\mathrm{L}(a(i), \delta) \cup \mathrm{L}\left(\delta, b_{m}(j)\right)
$$

and, to top it all off, $\gamma_{0}<\mathrm{L}(a(i), \delta)$ means that the $\mathrm{L}(a(i), \delta)$ part of $\mathrm{L}\left(a(i), b_{m}(j)\right)$ won't contain oscillations on the weight functions due to how we picked $\gamma_{0}$.

Now the proof is complete.

This is the main result about the oscillation of the lower trace function, created by Moore to construct the $\mathrm{L}$ space. An L space is a regular Hausdorff space that is hereditarily Lindelöf and is not hereditarily separable. In 1980, Zenor proved in [Zen80] that the existence of strong $\mathrm{L}$ spaces is linked to the existence of strong $\mathrm{S}$ spaces. Later, Todorcevic proved that with $M A_{\aleph_{1}}$, there are no strong $\mathrm{L}$ spaces nor $\mathrm{S}$ spaces and that it is consistent with $M A_{\aleph_{1}}$ that there are no S spaces and that there is an L space. Only in 2005, Moore showed that there is an L space with no use of additional axioms in [Moo06]. We will break down his construction on this chapter.

\subsection{The $o$ function}

To enter the realm of topology, we need a topological space. We will build things from $\mathbb{T}^{\omega_{1}}$, where $\mathbb{T}$ is the unit circle $\{z \in \mathbb{C}:|z|=1\}$. To start things off, we will state the old Kronecker's Approximation Theorem in a different version. See [Kro84] for the demonstration and more details. Then, with Kronecker's result and 3.1.3 in mind, we will make a definition 
and prove a proposition to link the things we have right now with topology.

Definition 3.2.1. Given a set $A \subset \mathbb{T}$, we say that $A$ is rationally independent when the set $\left\{x \in[-\pi, \pi]: e^{i x} \in A\right\}$ is linearly independent in $\mathbb{R}$ seen as a vector space over $\mathbb{Q}$.

Theorem 3.2.2 (Kronecker). Let $\left\langle z_{i}: i<k\right\rangle$ be rationally independent points of $\mathbb{T}$. For every $\epsilon>0$ there is $n_{\epsilon}<\omega$ such that, given $u \in \mathbb{T}^{k}$ and $v \in \mathbb{T}^{k}$, there is an $m<n_{\epsilon}$ such that for all $i<k$,

$$
\left|u_{i} z_{i}^{m}-v_{i}\right|<\epsilon
$$

Definition 3.2.3. Given $\left\langle z_{\alpha}: \alpha<\omega_{1}\right\rangle$ a sequence of rationally independent points of $\mathbb{T}$, for two countable ordinals $\alpha<\beta$, we define:

$$
o(\alpha, \beta)=z_{\alpha}^{\mathrm{osc}}(\alpha, \beta)+1
$$

Now, for the remainder of this work, we will just assume that we have a fixed sequence $\left\langle z_{\alpha}: \alpha<\omega_{1}\right\rangle$ of rationally independent points of $\mathbb{T}$, just like we did with the $C$-sequences in the first chapter.

Proposition 3.2.4. Let $\mathcal{A} \subset\left[\omega_{1}\right]^{k}$ and $\mathcal{B} \subset\left[\omega_{1}\right]^{l}$ be uncountable sets with pairwise disjoint elements. Given a sequence $\left\langle U_{i}: i<k\right\rangle$ of open neighborhoods of $\mathbb{T}$ and a function $\phi: k \longrightarrow l$, there are $a \in \mathcal{A}$ and $b \in \mathcal{B}$ with $a<b$ and for every $i<k$,

$$
o(a(i), b(\phi(i))) \in U_{i}
$$

Proof. Due to T's topology, we can safely assume that each $U_{i}$ is a radius $\epsilon$ ball centered on some $v_{i}$, with some fixed $\epsilon>0$. So, with Kronecker's theorem, for each $a \in \mathcal{A}$ we can find a $n_{\epsilon}$ for the sequence $\left\langle z_{a(i)}: i<k\right\rangle$ and the fixed $\epsilon$ of before. But since $\mathcal{A}$ is uncountable and there are only countable many possibilities for $n_{\epsilon}$, there is a specific $n_{\epsilon}$ with uncountable many elements of $\mathcal{A}$ that fall on it. So we can assume that $\mathcal{A}$ is actually this subset. In other words, we can safely assume that all $a \in \mathcal{A}$ have the same $n_{\epsilon}$.

Having all of those assumptions, we can now use 3.1.3 to get an $a \in \mathcal{A}$ and a $\left\langle b_{m}\right.$ : $m<n_{\epsilon}\left\langle\right.$ in $\mathcal{B}$ such that for all $i<k, j<l$ and $m<n_{\epsilon}$,

$$
\begin{gathered}
a<b_{m} \\
\operatorname{Osc}_{\mathrm{L}}\left(a(i), b_{m}(j)\right)=\operatorname{osc}_{\mathrm{L}}\left(a(i), b_{0}(j)\right)+m .
\end{gathered}
$$

Setting $u_{i}=o\left(a(i), b_{0}(\phi(i))\right)$ for every $i<k$, by Kronecker's theorem we can conclude that there is $m<n_{\epsilon}$ such that for every $i<k,\left|u_{i} z_{a(i)}^{m}-v_{i}\right|<\epsilon$, which means that $o\left(a(i), b_{m}(\phi(i))\right) \in U_{i}$. 
At last, one more definition before we start. This is more a change of notation to make things easier to use in fact.

Definition 3.2.5. For every $\beta<\omega_{1}$, define $w_{\beta}: \omega_{1} \longrightarrow \mathbb{T}$ by putting $w_{\beta}(\alpha)=o(\alpha, \beta)$ if $\alpha<\beta$. Otherwise, put $w_{\beta}(\alpha)=1$. Let's call $\mathcal{L}=\left\{w_{\beta}: \beta<\omega_{1}\right\}$ the set of all such functions viewed as a subspace of $\mathbb{T}^{\omega_{1}}$, and given some uncountable set $X \subset \omega_{1}$, let's call $\mathcal{L}_{X}=\left\{w_{\beta} \uparrow_{X}: \beta \in X\right\}$ viewed as a topological subspace of $\mathbb{T}^{X}$.

\subsection{The construction}

Now, let's do an analysis of both $\mathcal{L}$ and $\mathcal{L}_{X}$ on how and why we chose those last definitions. Why the need to extend the $o$ function with a constant function? Notice that pretty much everything we prove for $\mathcal{L}_{X}$ is also true for $\mathcal{L}$, since we can just take $X=\omega_{1}$.

But first, for memory's sake, let's recall what an L space is.

Definition 3.3.1. Let $X$ be a regular Hausdorff topological space. If it is hereditarily Lindelöf and not hereditarily separable, we call it an $\mathrm{L}$ space.

Proposition 3.3.2. $\mathcal{L}_{X}$ is not separable.

Proof. Let's take any countable set $D \subset \mathcal{L}_{X}$ and see that it can not be dense. First, let's call $D^{\prime}=\left\{\xi \in \omega_{1}: w_{\xi}\left\lceil_{X} \in D\right\}\right.$. Since $D$ is countable, then so is $D^{\prime}$, which means there is some $\delta<\omega_{1}$ in $X$ such that $D^{\prime}<\delta$. So, given the definition of each $w_{\xi}$, we have that for all $\xi \in D^{\prime}, w_{\xi}(\delta)=1$. This means that $D$ does not intercept the open set where the $\delta$ 's entry is a $1 / 2$ ball around $i$ and all other entries are $\mathbb{T}$. This proves that $\mathcal{L}_{X}$ is not separable.

In fact, there is a little more to this demonstration. We can use it to go a little deeper.

Proposition 3.3.3. $\mathcal{L}_{X}$ is hereditarily non separable.

Proof. Let's take an uncountable subspace $S$ of $\mathcal{L}_{X}$. Let's say $Y=\left\{\xi \in \omega_{1}: w_{\xi} \uparrow_{X} \in S\right\}$. Notice that $S \neq \mathcal{L}_{Y}$ due to the different domain of the functions. It goes the same way as before. Just take a countable subset of $S$ and the rest goes exactly how it went.

You might have guessed where this is going. It looks like $\mathcal{L}_{X}$ is our L space since we already proved it's hereditarily non separable and it's clearly regular due to compactness. We only have to prove it is also hereditarily Lindelöf now. You are right, $\mathcal{L}_{X}$ is an L space. To prove it, we need an auxiliary result first. 
Lemma 3.3.4. Given $X$ and $Y$ uncountable subsets of $\omega_{1}$ such that $X \cap Y$ is countable, we have that there are no continuous injections from any uncountable subspace of $\mathcal{L}_{X}$ into $\mathcal{L}_{Y}$.

Proof. Suppose there is such an injection $g$. We can say that $g$ is such that $g\left(w_{\alpha}\right)=w_{f(\alpha)}$ where $f$ is an injection from $X_{0}$ into $Y$, where $X_{0} \subset X$ is uncountable. Since $X_{0} \backslash Y$ is uncountable, we can take uncountable sequences $\left\langle\beta_{\xi}: \xi<\omega_{1}\right\rangle$ in $X_{0} \backslash Y$ and $\left\langle\zeta_{\xi}: \xi<\omega_{1}\right\rangle$ in $Y$. Not only that, we want them to be such that $f\left(\beta_{\xi}\right)>\zeta_{\xi}$ and, if $\xi<\xi^{\prime}$, then $\beta_{\xi}<\zeta_{\xi^{\prime}}$. Such sequence can easily be constructed with transfinite recursion by just taking the next two points that fit these criteria. Let $\Xi \subset \omega_{1}$ be uncountable such that for some open set $V \subset \mathbb{T}, g\left(w_{\beta_{\xi}}\right)\left(\zeta_{\xi}\right) \notin \bar{V}$ for all $\xi \in \Xi$. Now, for each $\xi \in \Xi$, consider the following set:

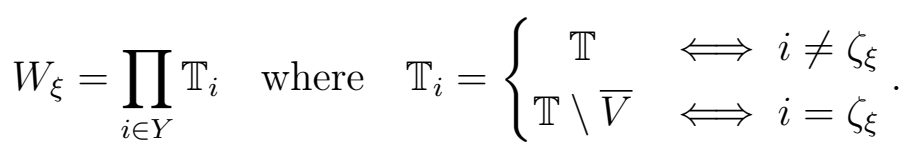

You can see that $W_{\xi}$ is an open set and thus, since $g$ is continuous by hypothesis, there is an open basic neighborhood $U_{\xi} \subset g^{-1}\left(W_{\xi}\right)$ of $w_{\beta_{\xi}}$. Now, for each $\xi \in \Xi$, since $U_{\xi}$ is an open basic set, it is a product with only finite entries different than $\mathbb{T}$, so let's say $a_{\xi}$ is the set of those entries, and let's put $\mathcal{E}=\left\{a_{\xi} \in X^{<\omega}: \xi \in \Xi\right\}$. Since $\mathcal{E}$ is uncountable, there is $k<\omega$ such that there are uncountable many $a_{\xi}$ 's with size $k$. Let's say $\mathcal{E}^{\prime}=\left\{a_{\xi} \in \mathcal{E}:\left|a_{\xi}\right|=k\right\}$. Now $\mathcal{E}^{\prime}$ is an uncountable set of finite sets of the same size $k$. Next, we are going to apply the $\Delta$-system lemma to it, finding us an uncountable set $\Xi^{\prime} \subset \Xi$ such that $\left\{a_{\xi}: \xi \in \Xi^{\prime}\right\}$ is a $\Delta$-system with a root $a$. Also, since $a_{\xi} \backslash a$ must be different for each different $\xi \in \Xi^{\prime}, \Xi^{\prime}$ is uncountable and there are only countable many ordinals below $a$, there must be uncountable many $\xi \in \Xi^{\prime}$ such that $a<a_{\xi} \backslash a$. Let's call $\Xi^{\prime \prime}$ the set of those $\xi$ 's. Now, since $\mathbb{T}$ is second countable, let's fix a countable basis $\mathcal{U}$ of it. For each $i<k$, each $\xi \in \Xi^{\prime \prime}$ and $U \in \mathcal{U}$, consider the set $S_{\xi}(U, i)=\left\{w \in \mathcal{L}_{X}: w\left(a_{\xi}(i)\right) \in U\right\}$. Notice that for each $w \in \mathcal{L}_{X}, i<k$ and $\xi \in \Xi^{\prime \prime}$, there are $U \in \mathcal{U}$ such that $w \in S_{\xi}(U, i)$. So, given a fixed $i<k$, there are uncountable many $\xi \in \Xi^{\prime \prime}$ but only countable many $U \in \mathcal{U}$ such that $w_{\beta_{\xi}} \in S_{\xi}(U, i)$. Not only that, but by the definition of $a_{\xi}$ and $\Xi$, we can make $U$ small enough so that $S_{\xi}(U, i)$ is a subset of $U_{\xi}$. So, fixing $i=0$, we can pick a small enough $U_{0} \in \mathcal{U}$ where there are uncountable many $\xi \in \Xi^{\prime \prime}$ such that $w_{\beta_{\xi}} \in S_{\xi}\left(U_{0}, 0\right)$ and let's put $\Xi_{0}^{\prime \prime}=\left\{\xi \in \Xi^{\prime \prime}: w_{\beta_{\xi}} \in S_{\xi}\left(U_{0}, 0\right)\right\}$. Then, for $i=1$, we can find an open basic $U_{1}$ such that $\Xi_{1}^{\prime \prime}=\left\{\xi \in \Xi_{0}^{\prime \prime}: w_{\beta_{\xi}} \in S_{\xi}\left(U_{1}, 1\right)\right\}$ is also uncountable. Doing this process till $i=k-1$ nets us an uncountable set $\Xi^{\prime \prime \prime}=\left\{\xi \in \Xi^{\prime \prime}:(\forall i<k)\left(w_{\beta_{\xi}} \in S_{\xi}\left(U_{i}, i\right)\right)\right\}$. Now, for all $\xi \in \Xi^{\prime \prime \prime}$, either $\beta_{\xi}<f\left(\beta_{\xi}\right)$ or $\beta_{\xi}>f\left(\beta_{\xi}\right)$. Again, finite possibilities for uncountable many $\xi$ 's. Pick the inequality that has uncountable many occurrences and call $\Xi^{\prime \prime \prime \prime}$ the family of such $\xi$ 's. Finally, since $\left|a_{\xi}\right|=k$ for all $\xi \in \Xi^{\prime \prime \prime \prime}$, we know that there are finite many possibilities 
for $\left|\zeta_{\xi} \cap a_{\xi}\right|$. With the same argument, we can take an uncountable $\Xi^{*} \subset \Xi^{\prime \prime \prime \prime}$ such that $\left|\zeta_{\xi} \cap a_{\xi}\right|=\left|\zeta_{\xi^{\prime}} \cap a_{\xi^{\prime}}\right|$ for all $\xi \in \Xi^{*}$ and $\xi^{\prime} \in \Xi^{*}$. Notice that this quantity must be less than $k$ because $\left\{\max a_{\xi}: \xi \in \Xi^{*}\right\}$ is unbounded in $\omega_{1}$ due to uncountably many different $a_{\xi}$ and $\zeta_{\xi}<\omega_{1}$. It is also important to note that $a<\zeta_{\xi}$ because otherwise it means the whole uncountable sequence $\zeta_{\xi}$ is less than $\max a$, which can't be the case.

To summarize, we have an uncountable set $\Xi^{*} \subset \Xi$, a $k<\omega, a_{\xi} \in[X]^{k}$ for every $\xi \in \Xi^{*}$, an open basic $U_{i}$ of $\mathbb{T}$ for all $i<k$ and some fixed $j \leq k$ such that:

1. $\left\{a_{\xi}: \xi \in \Xi^{*}\right\}$ is a $\Delta$-system with root $a$;

2. $a<a_{\xi} \backslash a$ for all $\xi \in \Xi^{*}$;

3. $w_{\beta_{\xi}} \in\left\{w \in \mathcal{L}_{X}:(\forall i<k)\left(w\left(a_{\xi}(i)\right) \in U_{i}\right)\right\} \subset U_{\xi}$ for all $\xi \in \Xi^{*}$;

4. either $\beta_{\xi}<f\left(\beta_{\xi}\right)$ for all $\xi \in \Xi^{*}$ or $\beta_{\xi}>f\left(\beta_{\xi}\right)$ for all $\xi \in \Xi^{*}$;

5. $\left|\zeta_{\xi} \cap a_{\xi}\right|=j$ for all $\xi \in \Xi^{*}$.

Up to this point on this demonstration, things might have been looking excessive and pointless. From now on, things will start to fit together and make sense. Hopefully.

Let's define the two following families:

$$
\begin{gathered}
\mathcal{A}=\left\{a_{\xi} \cup\left\{\zeta_{\xi}\right\} \backslash a \in\left[\omega_{1}\right]^{k+1-j}: \xi \in \Xi^{*}\right\} \\
\mathcal{B}=\left\{\left\{\beta_{\xi}, f\left(\beta_{\xi}\right)\right\} \in\left[\omega_{1}\right]^{2}: \xi \in \Xi^{*}\right\}
\end{gathered}
$$

The idea is to apply 3.2.4 to these families. Let's clarify a few things about their weirdness. $\mathcal{A}$ has to do with property 5 , which actually means that $a_{\xi}(j)<\zeta_{\xi}<a_{\xi}(j+1)$ for all $\xi \in \Xi^{*}$. Also, now that we took out $a, \mathcal{A}$ is indeed a family of pairwise disjoint sets. Also note that $a$ does not include $a_{\xi}(j+1)$ because $a<\zeta_{\xi}$ as discussed before. In the worse case, $a$ might include $a_{\xi}(j)$, but that won't be a problem. $\mathcal{B}$ has to do with property 4 as one might expect.

Now, to fully apply 3.2.4, one also needs a finite sequence of open neighborhoods of $\mathbb{T}$. That's when property 3 comes in handy with our fixed $U_{i}$ for each $i<k$. Since the elements of $\mathcal{A}$ have $k+1-j=n<k$ elements, we don't need all $U_{i}$ at the same time. By looking at $\mathcal{A}$ 's definition, we are only going to take those for which $a_{\xi}(i) \notin a$, of course. We also need another one to fit the last element $\zeta_{\xi}$ in all of them. That one will be $V$, the 
one open set we took arbitrarily at the beginning to define $\Xi$. For last, we need a function $\phi: n \longrightarrow 2$. We will make is based on property 4 in the following way: if the inequality is $<$, then $\phi(j+1-|a|)=1$ and $\phi(x)=0$ for all other $x$ in $n$. If the inequality is $>$, then $\phi(j+1-|a|)=0$ and $\phi(x)=1$ for all other $x$. Now we can fully apply the theorem and we used every weird detail we called.

So, the theorem gives us $a \in \mathcal{A}$ and $b \in \mathcal{B}$ such that $a<b$ and $o(a(i), b(\phi(i))) \in U_{i}$. In our case, it gives us $\xi$ and $\xi^{\prime}$ in $\Xi^{*}$ such that

6. $w_{\beta_{\xi^{\prime}}}\left(a_{\xi}(i)\right)=o\left(a_{\xi}(i), \beta_{\xi^{\prime}}\right) \in U_{i}$;

7. $g\left(w_{\beta_{\xi^{\prime}}}\right)=w_{f\left(\beta_{\xi^{\prime}}\right)}\left(\zeta_{\xi}\right)=o\left(\zeta_{\xi}, f\left(\beta_{\xi^{\prime}}\right)\right) \in V$.

You get these because of the $o(a(i), b(\phi(i))) \in U_{i}$ part of 3.2.4, the way we defined $\phi$ and property 3. To end this proof, notice that 6 means $w_{\beta_{\xi^{\prime}}} \in U_{\xi}$ and 7 implies that $g\left(w_{\beta_{\xi^{\prime}}}\right) \notin W_{\xi}$, which contradicts our choice of $U_{\xi}$.

Theorem 3.3.5. For every uncountable $X \subset \omega_{1}, \mathcal{L}_{X}$ is hereditarily Lindelöf.

Proof. Let's suppose that $\mathcal{L}_{X}$ is not hereditarily Lindelöf. We are first going to prove that then it would contain an uncountable discrete subspace. So, let's take $S \subset \mathcal{L}_{X}$ a non Lindelöf subspace with an open covering $\mathcal{C}$ that does not admits a countable subcovering. Define recursively a sequence $\left\langle x_{\alpha}: \alpha<\omega_{1}\right\rangle$ of points in $S$ and $\left\langle V_{\alpha}: \alpha<\omega_{1}\right\rangle$ of open sets in $\mathcal{C}$ such that $x_{\alpha} \notin \bigcup\left\{V_{\xi}: \xi<\alpha\right\}$, for all $\alpha<\beta<\omega_{1}, V_{\alpha} \cap V_{\beta}=\varnothing$ and $x_{\alpha} \in V_{\alpha} \backslash \overline{\left\{x_{\xi}: \xi<\alpha\right\}}$. Note that $\overline{\left\{x_{\xi}: \xi<\alpha\right\}}$ is countable, and thus we can always select $x_{\alpha}$ from $V_{\alpha} \backslash \overline{\left\{x_{\xi}: \xi<\alpha\right\}}$. It is countable because each point $x_{\xi}$ that forms it is of the form $w_{\beta} \uparrow_{X}$, and since there are countable many $x_{\xi}$ 's, this means that there is some $\gamma \in X$ such that $w_{\beta}(\gamma)=1$ for all $w_{\beta}$ in $\left\{x_{\xi}: \xi<\alpha\right\}$. This means that they vary only in countable many coordinates and thus, their closure can only contain countable many points. Of course, by the way we built it, $\left\{x_{\alpha}: \alpha<\omega_{1}\right\}$ is discrete. Now, to make things easier, let's rename this set for $D=\left\{w_{\alpha} \Upsilon_{X}: \alpha \in A\right\}$, where $A \subset X$ is uncountable.

Now, since $D$ is discrete, for each $w_{\alpha} \Upsilon_{X} \in D$, there is a basic open set $U_{\alpha}=\prod\left\{V_{\alpha, \xi}\right.$ : $\xi<X\}$ that isolates $w_{\alpha} \Upsilon_{X}$ from the rest of $D$. Since $U_{\alpha}$ is a basic open, there is a finite set $F_{\alpha} \subset X$ of coordinates such that $U_{\alpha, \xi} \neq \mathbb{T}$ if and only if $\xi \in F_{\alpha}$. So, applying the $\Delta$-system lemma, we can find an uncountable subset $A^{\prime} \subset A$ such that for all $\alpha<\beta$ in $A^{\prime}, F_{\alpha} \cap F_{\beta}=F$.

Now, for each $\xi \in F$, there are only countable many possibilities for $U_{\alpha, \xi}$ if we consider that $\mathbb{T}$ is second countable. With an argument similar to the one we used in 3.3.4, we can 
find an uncountable $A^{\prime \prime} \subset A^{\prime}$ such that, for each $\xi \in F$, we have that $U_{\alpha, \xi}=U_{\beta, \xi}=V_{\xi}$ for all $\alpha \in A^{\prime \prime}$ and $\beta \in A^{\prime \prime}$. Since $D$ is still discrete, we have to remember that for each two ordinals $\alpha$ and $\beta$ in $A^{\prime \prime}$, the sets $U_{\alpha}$ and $U_{\beta}$ are still disjoints, thus, it means that the $\xi$ that makes $w_{\beta}(\xi) \notin U_{\xi, \alpha}$ is in $F_{\alpha} \backslash F$.

Now, we will construct an uncountable sequence by recursion. Take an arbitrary $\alpha_{0} \in A^{\prime \prime}$. Now, given $\alpha_{\xi}$ defined for all $\xi<\gamma$ such that

$$
\bigcup_{\beta<\xi}\left(\left\{\alpha_{\beta}\right\} \cup F_{\alpha_{\beta}} \backslash F\right) \cap\left(\left\{\alpha_{\xi}\right\} \cup F_{\alpha_{\xi}} \backslash F\right)=\varnothing
$$

take $\alpha_{\gamma} \in A^{\prime \prime}$ such that

$$
\bigcup_{\xi<\gamma}\left(\left\{\alpha_{\xi}\right\} \cup F_{\alpha_{\xi}} \backslash F\right) \cap\left(\left\{\alpha_{\gamma}\right\} \cup F_{\alpha_{\gamma}} \backslash F\right)=\varnothing,
$$

which exists because $A^{\prime \prime} \backslash\left\{\alpha_{\xi}: \xi<\gamma\right\}$ is uncountable. So, with that sequence, we can define ourselves a set $W=\bigcup\left\{\left\{\alpha_{\xi}\right\} \cup F_{\alpha_{\xi}} \backslash F: \xi<\omega_{1}\right\}$, which is a set such that $\mathcal{L}_{W}$ has $\left\{\alpha_{\xi}: \xi<\omega_{1}\right\}$ as a discrete subspace. This happens because $\mathcal{L}_{W}$ has $\left\{\alpha_{\xi}: \xi<\omega_{1}\right\}$ and all it's coordinates that gives them neighborhoods disjoints from each other. Now all we have to do is split it into two uncountable disjoint sets $Y$ and $Z$ such that $\mathcal{L}_{Y}$ and $\mathcal{L}_{Z}$ each contain a discrete subspace. We do this by splitting $\left\{\alpha_{\xi}: \xi<\omega_{1}\right\}$ in two uncountable sequences and giving each sequence and it's coordinates that isolate them to each set.

This way we can easily make a bijection from $\mathcal{L}_{Y}$ 's discrete into $\mathcal{L}_{Z}$ 's discrete because they have the same cardinality, and since every bijection from a discrete space into another discrete space is continuous, we end up contradicting lemma 3.3.4.

And there you have it. $\mathcal{L}_{X}$ is an $\mathrm{L}$ space. 


\section{Chapter 4}

\section{Applications in Model Theory}

This technique has applications outside the realm of set theory and general topology too. One of the first applications by Todorcevic was in model theory, as showcased in [Tod87]. Also, since last chapter was about oscillations on the lower trace function, this one will be featuring oscillations on the upper trace function instead.

\subsection{Oscillations on the upper trace}

When we were talking about the oscillations on the lower trace on the last chapter, we mentioned that the oscillations on the upper trace are more complicated to define because they are actually the oscillation of two sets instead of two functions. To make it work, we need to define functions that make it so the definition yields what we want.

Definition 4.1.1. Given $\alpha<\beta$ countable ordinals, let's define two functions $f_{\alpha}$ and $f_{\beta}$ from $\operatorname{Tr}\left(\Delta_{0}(\alpha, \beta), \alpha\right) \cup \operatorname{Tr}\left(\Delta_{0}(\alpha, \beta), \beta\right)$ into 2 in the following manner:

- If $\xi \in \operatorname{Tr}\left(\Delta_{0}(\alpha, \beta), \alpha\right)$, then $f_{\alpha}(\xi)=1$. Otherwise, $f_{\alpha}(\xi)=0$;

- If $\xi \in \operatorname{Tr}\left(\Delta_{0}(\alpha, \beta), \beta\right)$, then $f_{\beta}(\xi)=1$. Otherwise, $f_{\beta}(\xi)=0$.

Notice that because of the definition of the upper trace, $\left(\operatorname{Tr}\left(\Delta_{0}(\alpha, \beta), \alpha\right) \cap \operatorname{Tr}\left(\Delta_{0}(\alpha, \beta), \beta\right)\right)$ is an initial segment of both $\operatorname{Tr}\left(\Delta_{0}(\alpha, \beta), \alpha\right)$ and $\operatorname{Tr}\left(\Delta_{0}(\alpha, \beta), \beta\right)$. So, what this oscillation is really counting is in how many pieces does $\operatorname{Tr}\left(\Delta_{0}(\alpha, \beta), \beta\right)$ splits $\operatorname{Tr}\left(\Delta_{0}(\alpha, \beta), \alpha\right)$, that is, how many maximal intervals without points from $\operatorname{Tr}\left(\Delta_{0}(\alpha, \beta), \beta\right)$ does $\operatorname{Tr}\left(\Delta_{0}(\alpha, \beta), \alpha\right)$ has. 


$$
o s c(\alpha, \beta)=3
$$

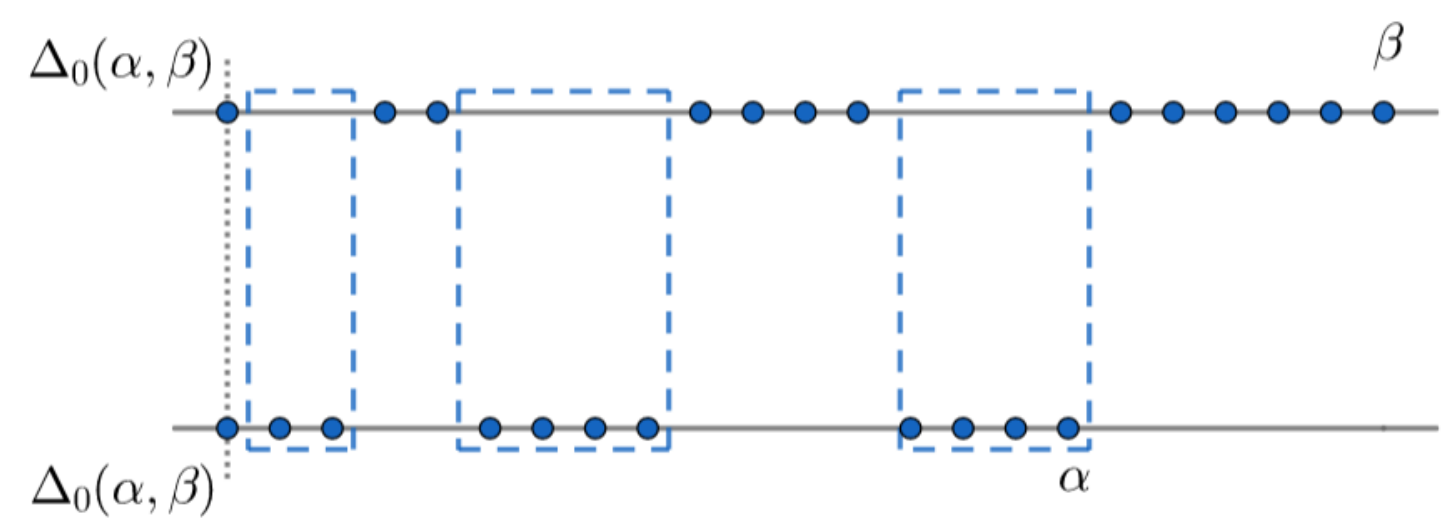

Now, here are the formal definitions:

Definition 4.1.2. Given $\alpha<\beta$ countable ordinals, we will write $\operatorname{Osc}_{\operatorname{Tr}}(\alpha, \beta)$ and $\operatorname{osc}_{\operatorname{Tr}}(\alpha, \beta)$ to denote the following:

- $\operatorname{Osc}_{\operatorname{Tr}}(\alpha, \beta)=\left\{\xi_{i+1} \in \operatorname{Tr}(\Delta(\alpha, \beta), \alpha) \cup \operatorname{Tr}(\Delta(\alpha, \beta), \beta): f_{\alpha}\left(\xi_{i}\right) \leq f_{\beta}\left(\xi_{i}\right)\right.$ and $f_{\alpha}\left(\xi_{i+1}\right)>$ $\left.f_{\beta}\left(\xi_{i+1}\right)\right\}$;

- $\operatorname{osc}_{\operatorname{Tr}}(\alpha, \beta)=\left|\operatorname{Osc}_{\operatorname{Tr}}(\alpha, \beta)\right|$.

Just like the main theorem about the oscillations on the lower trace, this result is also complicated and difficult to prove. But after you get accustomed to this type of demonstration, this one gets easier too. The result is pretty much the same, but for the upper trace instead.

Theorem 4.1.3. Given an uncountable set $\mathcal{A} \subset \omega_{1}$ and $n<\omega$, there exists $l<\omega$ such that for all $k<n$, there are $\alpha<\beta$ in $\mathcal{A}$ such that

$$
\operatorname{Osc}_{\operatorname{Tr}}(\alpha, \beta)=l+k
$$

Proof. Let's take a sequence $\left\langle\mathcal{M}_{i}: i<n+1\right\rangle$ where each $\mathcal{M}_{i}$ is an $\in$-chain of countable elementary submodels of $H\left(\omega_{1}^{+}\right)$containing all the relevant objects we need such that, for all $i<n$, the minimal element of $\mathcal{M}_{i+1}$ contains $\mathcal{M}_{i}$ as an element. Putting $M_{n}$ the minimal element of $\mathcal{M}_{n}$ and $\delta_{n}=M_{n} \cap \omega_{1}$, let's take some $\beta \in \mathcal{A}$ such that $\beta>\delta_{n}$. Now, consider that $\mathcal{M}_{n-1} \in M_{n}$ and that $\mathcal{M}_{n-1}$ is uncountable, which means $\mathcal{M}_{n-1} \cap M_{n} \neq \varnothing$ by elementarity. Since $\mathcal{M}_{n-1}$ is an $\in$-chain of countable elements, then $\mathcal{M}_{n-1} \cap M_{n}$ is an initial segment of 
$\mathcal{M}_{n-1}$. Now, consider the set $\left\{\xi \in \omega_{1}:\left(\exists M \in \mathcal{M}_{n-1}\right)\left(\xi=M \cap \omega_{1}\right)\right\}$. With elementarity, this set belongs to $M_{n}$ and thus is unbounded in $\delta_{n}$, which means we can actually take some $M_{n-1} \in \mathcal{M}_{n-1} \cap M_{n}$ with $M_{n-1} \cap \omega_{1}=\delta_{n-1}$ such that $\mathrm{L}\left(\delta_{n}, \beta\right)<\delta_{n-1}<\delta_{n}$. Repeating this argument, we can find some $M_{n-2} \in \mathcal{M}_{n-2} \cap M_{n-1}$ with $M_{n-2} \cap \omega_{1}=\delta_{n-2}$ such that $\mathrm{L}\left(\delta_{n-1}, \delta_{n}\right)<\delta_{n-2}<\delta_{n-1}$ and so on. This will give us an $\in$-chain $\left\langle M_{i}: i<n+1\right\rangle$ of countable elementary submodels of $H\left(\omega_{1}^{+}\right)$containing everything relevant such that $\mathrm{L}\left(\delta_{i+1}, \delta_{i+2}\right)<\delta_{i}<$ $\delta_{i+1}$ for all $i<n$ if we set $\delta_{n+1}=\beta$, with $M_{i} \cap \omega_{1}=\delta_{i}$ for all $i<n+1$.

Let $\xi \in C_{\delta_{0}}$ be above $\mathrm{L}\left(\delta_{0}, \beta\right)$. By 1.1.3, $\delta_{0} \in \operatorname{Tr}(\xi, \beta)$. Also, because $\mathrm{L}\left(\delta_{1}, \delta_{2}\right)<\delta_{0}$, we conclude that $\delta_{1} \in \operatorname{Tr}\left(\delta_{0}, \beta\right) \subset \operatorname{Tr}(\xi, \beta)$. In fact, with the same argument, we get that $\delta_{i} \in \operatorname{Tr}(\xi, \beta)$ for all $i \leq n+1$. Because $\xi \in C_{\delta_{0}}$ and the definition of the upper trace, $\delta_{0}$ is actually the last step before $\xi$ in $\operatorname{Tr}(\xi, \beta)$. With these, consider $t=\varrho_{0}(\xi, \beta)$ and $t_{i}=\varrho_{0}\left(\delta_{i}, \beta\right)$ for all $i<n+1$. Of course, we have that $t_{n} \sqsubset t_{n-1} \sqsubset \cdots \sqsubset t_{1} \sqsubset t_{0} \sqsubset t=t_{0} \wedge\left|C_{\delta_{0}} \cap \xi\right|$, where $x \sqsubset y$ means that $x \subset y$ and that $x$ is an initial segment in the ordering of $y$. Now, consider the following set:

$$
\mathcal{A}_{0}=\left\{\gamma \in \mathcal{A}: t=\varrho_{0}(\xi, \gamma) \text { and } \varrho_{0 \gamma} \uparrow_{\xi}=\varrho_{0 \beta} \Upsilon_{\xi}\right\}
$$

Clearly, $\beta \in \mathcal{A}_{0}$. Also, notice that $\mathcal{A}_{0} \in M_{0}$ because $\varrho_{0 \beta}$ is restricted to $\xi$, which means that $\mathcal{A}_{0}$ is uncountable. For each $\Sigma \subset \mathcal{A}_{0}$, let's make a tree ordered by $\sqsubseteq$ of the following things:

$$
\mathcal{T}_{\Sigma}=\left\{x \in\left[\omega_{1}\right]^{\leq|t|}:(\exists \alpha \in \Sigma)(x \sqsubseteq \operatorname{Tr}(\xi, \alpha))\right\}
$$

Let's say that a subset $\Sigma \subset \mathcal{A}_{0}$ is large if $\mathcal{T}_{\Sigma}$ contains a subtree $\mathcal{T}$ whose root is $\{\xi\}$ and, for each node $x$ with $|x|<|t|$, it either has an immediate successor or there is an $i<n+1$ such that there are uncountably many $\gamma<\omega_{1}$ such that $x \cup\{\gamma\} \in \mathcal{T}$ and $\varrho_{0}(\gamma, \alpha)=t_{i}$ for all $\alpha \in \Sigma$ such that $x \sqsubseteq \operatorname{Tr}(\xi, \alpha)$. We are going to prove that $\mathcal{A}_{0}$ is large.

We are going to do so by building the tree $\mathcal{T}$. Consider for each node $x$ and for each $i<n+1$ the set

$$
S_{x, i}=\left\{\gamma<\omega_{1}:\left(\forall \alpha \in \mathcal{A}_{0}\right)\left(x \sqsubseteq \operatorname{Tr}(\xi, \alpha) \longrightarrow \varrho_{0}(\gamma, \alpha)=t_{i}\right)\right\}
$$

Since we know the root must be $\{\xi\}$, then let's start analyzing that point. Since it's the beginning of the sequence, let's check the set $S_{\{\xi\}, 0}$. By the definition of $\mathcal{A}_{0}$, we get that $\delta_{0} \in S_{\{\xi\}, 0}$, and by the definition of $S_{\{\xi\}, 0}$, we get that $S_{\{\xi\}, 0} \in M_{0}$, which implies that $S_{\{\xi\}, 0}$ is uncountable. Good, so we can add $\{\xi\}$ and $\{\xi, \gamma\}$ for all $\gamma \in S_{\{\xi\}, 0}$ to $\mathcal{T}$. Since the whole traces of $\operatorname{Tr}\left(\xi, \delta_{i}\right)$ are present in $\mathcal{T}_{\mathcal{A}_{0}}$, we can go ahead and add all nods that go up from 
$\left\{\xi, \delta_{0}\right\}=\operatorname{Tr}\left(\xi, \delta_{0}\right)$ to $\operatorname{Tr}\left(\xi, \delta_{1}\right) \backslash\left\{\delta_{1}\right\}=x_{1}$ to our subtree. With the same argument as before, we get that $S_{x_{1}, 1}$ has $\delta_{1}$ and is uncountable, which lets us put all the nodes $x_{1} \cup\{\gamma\}$ for all $\gamma \in S_{x_{1}, 1}$. Again, we can add the nodes that go from $x_{1}$ up to $x_{2}=\operatorname{Tr}\left(\xi, \delta_{2}\right) \backslash\left\{\delta_{2}\right\}$ and add the nodes $x_{2} \cup\{\gamma\}$ for all $\gamma \in S_{x_{2}, 2}$, which is uncountable and has $\delta_{2}$. This way, $\mathcal{T}$ has a central stalk of all $x \sqsubseteq \operatorname{Tr}(\xi, \beta)$, with uncountable many branches growing from specific nodes. All we have to do now is analyze these nodes now. So, by the definition of $\mathcal{A}_{0}$, we get that these nodes are such that there is an $\alpha \in \mathcal{A}$ such that the walk from $\alpha$ to the node has $|t|$ many steps, which means we can add those steps going up to top to complete the tree.

Ok, now we have that $\mathcal{A}_{0}$ is large. Also notice that if we split it into countable many disjoint pieces, the uncountable pieces must be large because we can just take the nods in the $\mathcal{T}$ we built that also come from them and the new tree will also fit what we want. This way we can find a large $\Sigma \subset \mathcal{A}_{0}$ such that $\Sigma \in M_{0}$ and $\Delta_{0}(\alpha, \beta)$ is the same ordinal for all $\alpha \in \Sigma$. By the definition of $\mathcal{A}_{0}$, we get that this ordinal is greater than $\xi$, and because $\mathcal{A}_{0} \in M_{0}$, it must be less than $\delta_{0}$. Let's call this ordinal $\xi_{0}$. So, to reiterate, we have a large $\Sigma \in M_{0}$ and a $\xi<\xi_{0}<\delta_{0}$ such that $\Delta_{0}(\alpha, \beta)=\xi_{0}$ for all $\alpha \in \Sigma$. Let's look at $\operatorname{Tr}\left(\xi_{0}, \beta\right)$. Since $\xi_{0}<\delta_{0}<\max (\operatorname{Tr}(\xi, \beta) \backslash\{\beta\})$, we get that the first step of this walk will be the same as the walk from $\beta$ to $\xi$ and it will keep going down till $\delta_{0}$, where it will divert and go somewhere else instead of $\xi$. So if we put $s=\varrho\left(\xi_{0}, \beta\right)$, we get that $t_{n} \sqsubset t_{n-1} \sqsubset \cdots \sqsubset t_{0} \sqsubset s$.

Now, since the formula for knowing when a set is large is understood by $M_{0}$, let's take a subtree $\mathcal{T} \in M_{0}$ witnessing that $\Sigma$ is large. Since $\mathcal{T}$ is such that $\{\xi\}$ has uncountable many successors, we can pick one successor $\{\xi, \zeta\}$ such that $\xi_{0}<\zeta$. By 1.1.3, we get that for every $\alpha \in \Sigma, \operatorname{Tr}\left(\xi_{0}, \alpha\right)=\operatorname{Tr}\left(\xi_{0}, \zeta\right) \cup \operatorname{Tr}(\zeta, \alpha)$ and due to the definition of the subtree of large sets, $\varrho(\zeta, \alpha)=t_{0}$. Pick a branching node $x_{1} \sqsupset\{\xi, \zeta\}$ such that the immediate successors $x_{1} \cup\{\gamma\} \in \mathcal{T}$ are such that $\varrho_{0}(\gamma, \alpha)=t_{1}$ for all $\alpha \in \Sigma$ in which $x_{1} \sqsubseteq \operatorname{Tr}(\xi, \alpha)$. Let $l$ be the oscillation of the walks $\operatorname{Tr}\left(\xi_{0}, \zeta\right) \cup x_{1}=\operatorname{Tr}\left(\xi_{0}, \max x_{1}\right)$ and $\operatorname{Tr}\left(\xi_{0}, \beta\right)$. Now pick an arbitrary $k<n$. Looking at things from $M_{1}$ now, take a immediate successor $x_{1} \cup\left\{\gamma_{1}\right\}$ such that $\gamma_{1}>\delta_{1} \cap \operatorname{Tr}\left(\xi_{0}, \beta\right)$ and then choose a branch $x_{2}$ extending $x_{1} \cup\left\{\gamma_{1}\right\}$ whose full code $t_{2}$. Then, in $M_{2}$, take an immediate successor $x_{2} \cup\left\{\gamma_{2}\right\}$ such that $\gamma_{2}>\delta_{2} \cap \operatorname{Tr}\left(\xi_{0}, \beta\right)$ and then choose an extending branch $x_{3}$ of it such that satisfies $t_{3}$. Keep proceeding that way till the $k$-th step, which nets us some $x_{k} \cup\left\{\gamma_{k}\right\} \in \mathcal{T} \cap M_{k}$ such that $\gamma_{k}>\delta_{k} \cap \operatorname{Tr}\left(\xi_{0}, \beta\right)$. Now in $M_{k}$, we just pick some $\alpha \in \Sigma$ such that $x_{k} \cup\left\{\gamma_{k}\right\} \sqsubseteq \operatorname{Tr}(\xi, \alpha)$.

We arrived at the end of the demonstration. Consider the traces $\operatorname{Tr}\left(\xi_{0}, \alpha\right)$ and $\operatorname{Tr}\left(\xi_{0}, \beta\right)$. The oscillation points will be $\gamma_{1}, \cdots, \gamma_{k}$ and the ones in $\operatorname{Tr}\left(\xi_{0}, \zeta\right) \cup x_{1}=\operatorname{Tr}\left(\xi_{0}, \max x_{1}\right)$ and $\operatorname{Tr}\left(\xi_{0}, \beta\right)$, meaning that $\operatorname{osc}_{\operatorname{Tr}}(\alpha, \beta)=l+k$. 
And that was the hard part. Now we can show the applications of this theorem.

\subsection{Results}

Just like the L space, we will first need a way to link oscillations to something else before we can apply 4.1.3. And like the $\mathrm{L}$ space, this will be a function projecting oscillations onto other mathematical objects. In this particular case, we won't be applying it to topology, so the function this time will be fairly different. Let's get to it.

Definition 4.2.1. Take a function $\mathrm{osc}_{\mathrm{Tr}}^{*}: \omega_{1} \times \omega_{1} \longrightarrow \omega$ given by

$$
\operatorname{osc}_{\operatorname{Tr}}^{*}(\alpha, \beta)=\max \left\{m<\omega: 2^{m} \mid \operatorname{osc}_{\operatorname{Tr}}(\alpha, \beta)\right\}
$$

In this definition, the term $a \mid b$ stands for $a$ divides $b$. As you can guess, due to 4.1.3 we can find $\alpha$ and $\beta$ that satisfies $\operatorname{osc}_{\operatorname{Tr}}^{*}(\alpha, \beta)=k$ for every $k$ we want. Not only this function, but almost any projection we can make of the upper oscillation function will allow us to find entries that returns whatever value we want, which means that one can take more advantage of the upper trace oscillation than what will be shown here with this function. It's just that for this specific result, all we need is this simple function, so let's not over complicate things.

Lemma 4.2.2. Given $\mathcal{A} \subset \omega_{1}$ an uncountable set and $0<m<\omega$, there are $\alpha \in \mathcal{A}$ and $\beta \in \mathcal{A}$ such that

$$
\operatorname{Osc}_{\operatorname{Tr}}^{*}(\alpha, \beta)=m
$$

Proof. Let's apply 4.1 .3 for $\mathcal{A}$ and $2^{m+1}+1$. We get an $l<\omega$ such that we can find $\alpha_{k}$ and $\beta_{k}$ in $\mathcal{A}$ such that $\operatorname{osc}_{\operatorname{Tr}}\left(\alpha_{k}, \beta_{k}\right)=l+k$ for all $k<2^{m+1}+1$. This means we can satisfy any natural number from $l$ to $l+2^{m+1}$, which means that there are $k_{1}<k_{2}<2^{m+1}+1$ such that $\operatorname{osc}_{\operatorname{Tr}}\left(\alpha_{k_{1}}, \beta_{k_{1}}\right)$ and $\operatorname{osc}_{\operatorname{Tr}}\left(\alpha_{k_{2}}, \beta_{k_{2}}\right)$ consecutive multiples of $2^{m}$. If the first one is also a multiple of another greater power of 2 , this means that $\operatorname{osc}_{\operatorname{Tr}}\left(\alpha_{k_{1}}, \beta_{k_{1}}\right)=2^{m} C_{1}$, where $C_{1}$ is some even natural number. This means that $\operatorname{osc}_{\operatorname{Tr}}\left(\alpha_{k_{1}}, \beta_{k_{1}}\right)=2^{m} C_{2}$ has $2^{m}$ as the greater power of 2 that divides it because $C_{2}=C_{1}+1$ is odd. That means that either $\alpha_{k_{1}}$ and $\beta_{k_{1}}$ or $\alpha_{k_{2}}$ and $\beta_{k_{2}}$ will get the job done.

Our final result is in the realm of model theory. This result was first given by Todorcevic [Tod87] in 1987. Later, at 2007, Larson [Lar07] proved that this result couldn't be extended to an uncountable partition of $\omega_{1}$. 
Before we get to the result, we need a lemma from [Kun83].

Lemma 4.2.3. Given $\kappa>\omega$ a regular cardinal and, for every $\alpha<\kappa, C_{\alpha}$ a club in $\kappa$, then

$$
D=\left\{\gamma:(\forall \alpha<\gamma)\left(\gamma \in C_{\alpha}\right)\right\}
$$

is a club in $\kappa$. We say that $D$ is the diagonal intersection of $C_{\alpha}$.

It's not very hard to see that this is true. Since all $C_{\alpha}$ are $c l u b$, it follows that $D$ should have the necessary limit ordinals. We will omit this demonstration because it requires the demonstration of several other lemmas. For those interested, it's in chapter 2, lemma 6.14 in [Kun83].

Theorem 4.2.4. Every inner model $M$ of set theory that correctly understands $\omega_{1}$ can partition it into countably many pairwise-disjoint stationary sets in the class $V$ of all sets.

Proof. Since $M$ has $\omega_{1}$ as a whole, then it also contains a $C$-sequence $\left\{C_{\alpha}: \alpha<\omega_{1}\right\}$. That's the one we are going to use. This means that the oscillation function osc $\operatorname{Tr}_{\mathrm{Tr}}^{*}$ is also in $M$. For every $\alpha<\omega_{1}$ and $n<\omega$, consider the following set:

$$
S_{\alpha, n}=\left\{\beta>\alpha: \operatorname{osc}_{\operatorname{Tr}}^{*}(\alpha, \beta)=n\right\}
$$

Notice that this set also belongs to $M$ and that given a fixed $\alpha<\omega_{1}$, the sets $\left\{S_{\alpha, n}: n<\omega\right\}$ will split $\omega_{1}$ in countably many parts. It follows that we just need to prove that there is an $\alpha<\omega_{1}$ in which all $S_{\alpha, n}$ are stationary in $V$.

Let's suppose that for all $\alpha$ there is $n_{\alpha}<\omega$ such that $S_{\alpha, n_{\alpha}}$ is not stationary. Take $C_{\alpha}$ a $c l u b$ for every $\alpha<\omega_{1}$ such that $S_{\alpha, n_{\alpha}} \cap C_{\alpha}=\varnothing$, which exists since $S_{\alpha, n_{\alpha}}$ is not stationary. Let's take $D$ the diagonal intersection of these sets. For every $\alpha \in D$, there are countably many possibilities for $n_{\alpha}$, which means we can take an uncountable $D^{\prime} \subset D$ and $k<\omega$ such that for all $\alpha \in D^{\prime}, n_{\alpha}=k$. By 4.2.2, there $\alpha^{\prime}<\beta^{\prime}$ in $D^{\prime}$ such that

$$
\operatorname{OSc}_{\operatorname{Tr}}\left(\alpha^{\prime}, \beta^{\prime}\right)=k \text {. }
$$

By definition of $D$, we also have that $\beta^{\prime} \in C_{\alpha^{\prime}}$, which means that $\beta^{\prime} \notin S_{\alpha^{\prime}, k}$. It follows that $\operatorname{osc}_{\operatorname{Tr}}\left(\alpha^{\prime}, \beta^{\prime}\right) \neq k$.

And that's the main result based on oscillating the upper trace. 


\section{Appendix A}

\section{Mathematical Logic}

In order to understand the fundamentals of mathematics and all of this work, one needs to be fluent and comfortable when talking logic. In this appendix, we intend to give the reader some basic background in languages, syntax and propositions. Nothing presented here will be new. The point is to be didactic enough to provide a solid basis to anyone interested in this area to understand this document and possibly pursue deeper knowledge and understanding in other books, papers and other texts.

Talking about books and references, [Eps07] is a good book to start studying logic for any undergraduate student. It explains in detail the concepts presented here as well as other deeper concepts. [Rau06] is more compact and goes deeper in model theory and set theory as well, but of course it is more dense in exchange. It also has many exercises. Another interesting book is [EFT13], which delves deeper into model theory as well as logic. It's harder to separate model theory and logic actually, as both walk hand in hand.

So, without further ado, let's begin.

\section{A.1 Languages}

At it's core, logic is a part of linguistics. It's a way to make sense out of symbols, a way to see words, verbs and phrases on a sequence of symbols. Notice that I said that it's a way to make sense of them, not meaning. Sense and meaning are very different things. For example, I can say "flowers can talk" and you can understand it. It's something that makes sense, even though it is not true. Now, when I say "hair nine walk", you can't actually understand it because it doesn't make any sense at all. The nouns and verbs are out of 
English structure, so we can't even say if it's true or false at all! Let alone give meaning to it. Even worse is a phrase like "3!.304,7adf?l4", where you can't even identify things like nouns and verbs.

That's the concept we intend to flesh out here.

\section{A.1.1 Terms and sentences}

Definition A.1.1. A language is a sequence $\mathcal{L}=(C, F, R)$ where $C$ is a set of constant symbols, $F$ is a set of functional symbols and $R$ is a set of relational symbols. Also, we are free to use other symbols for variables and auxiliary symbols, such as (, [ and $\{$.

To understand each of these sets of symbols, we have to look at them in conjunction with each other, which makes explaining them separately a bit hard. Let's give an example first.

Example. Take $\mathcal{L}_{\mathbb{N}}=(\mathbb{N},\{+, \times\},\{=,<\})$. In this language, an example of phrase is $3+5=$ 8 , where $3+5$ and 8 are terms, our words or objects. In the phrase $x+y<(a \times b)+c$, the terms are $x+y$ and $(a \times b)+c$.

You see, in each phrase we are relating two terms with each other with one of the relation symbols. A phrase like $x, a+1$ or 0 make no sense on it's own, we have to compare them through $=$ or $<$. This is why we say that terms are objects and relation symbols are verbs. To make a phrase, we need two objects and a verb, at least most of the times.

We still don't know exactly what a term is, but it's easier to explain functional symbols first before going on to define term in a more formal way. Functional symbols are basically functions that accepts terms as inputs and outputs another term. In our example, the functions + and $\times$ take two terms and gives us one term back but not all functional symbols need to work like this.

Example. Take $\mathcal{L}_{V}=\left(\mathbb{R}^{2}, \mathbb{R} \cup\{+\},\{=\}\right)$ where we think of $\mathbb{R}^{2}$ as a vector space, with the normal addition operation + and, for each $\alpha \in \mathbb{R}$, the operation $\alpha$ means multiplying a vector with $\alpha$.

In this example, we have one binary function + and infinite unary functions, one for each real number. The same thing is true for relational symbols, they can also relate more than two or even only one term. The important thing to understand here is that functional 
symbols yields a term while relational symbols yield a phrase, or better yet, a sentence, an affirmation. You can't write things such as $(2=3)+1<x$ in $\mathcal{L}_{\mathbb{N}}$. So, in a more formal way... Definition A.1.2. Given a language $\mathcal{L}$, we say that a single variable or constant $\mathcal{L}$-symbol is a term with complexity 1 . Given a $n$-function $\mathcal{L}$-symbol $f\left(x_{1}, \cdots, x_{n}\right)$, we say that a term has complexity $k$ if it is of the form $f\left(t_{1}, \cdots, t_{n}\right)$, where $t_{i}$ is a term of complexity $k-1$ for each $i=1, \cdots, n$.

Now, for last, you can think of the constant symbols as any basic term that you want to highlight for some reason. In most cases, they are needed when the theory that the language is being used for has an axiom that needs a specific term doing something special. For example, in $\mathcal{L}_{\mathbb{N}}$ being used to describe our common arithmetics, we usually use constant symbols 0 and 1 to signify the neutral element for the addition and multiplication of natural numbers. The rest of the natural numbers don't really need a constant symbol for them on the language. Of course, we can define symbols for specific terms, for example,

$$
\begin{aligned}
& 2 \stackrel{\text { def }}{=} 1+1 ; \\
& 3 \stackrel{\text { def }}{=} 2+1 ; \\
& 4 \stackrel{\text { def }}{=} 3+1 ;
\end{aligned}
$$

but still, to define the language, we only need the 1 and the 0 . Another note on unnecessary symbols is about the symbol $=$. In truth, it is a symbol from propositional logic, so we don't need to add it to our languages because we already have it, as well as other well known logic symbols such as $\exists, \forall, \longrightarrow, \longleftrightarrow, \neg, \wedge$ and $\vee$, which we are going to explain more on the next session. But for now, let's focus on understanding the concept of a language.

So, these are all the formalities. The whole idea behind the language is to let us write something out of a set of symbols. Given $\mathcal{L}_{\mathbb{N}}=\{0,1,+, \times,<\}$ our new and optimized natural number arithmetics, we know how to write things out of it. We can write and read things like $(n \times x)+1<y,(a+1) \times(b+1)=0$, and we know that something like $+<a \times$ doesn't make any sense. You see, the point is to look at something written with these symbols and recognize if it makes sense or not.

Definition A.1.3. Given a language $\mathcal{L}$ and $R\left(x_{1}, \cdots, x_{n}\right)$ an $n$-relation symbol of $\mathcal{L}$, we say that a finite sequence $p$ of $\mathcal{L}$-symbols is a sentence if it is of the form $R\left(t_{1}, \cdots, t_{n}\right)$, where $t_{i}$ is a proper $\mathcal{L}$-term for all $i=1, \cdots, n$.

I'll repeat it again: the point is to look at something written in $\mathcal{L}$ and recognize if it is 
a sentence or not. As of yet, we can't give meaning to the sentences we write. For example, taking $\mathcal{L}_{\mathbb{N}}$ again, we know that both $1+1=1$ and $2+5=7$ are valid sentences, but we know that they are fundamentally different. For all we know, $2+5=7$ is true while $1+1=1$ is not. But how can we conclude this with all the ideas presented so far? Even worse, what it means for a sentence to be true or false? We haven't defined any of this. And that's because interpretation, meaning and falsehood are not concepts on the language's domain.

So for now, we will give more examples of languages and sentences. We will use dots to represent where each term goes on each function and relation symbol.

Example. $\mathcal{L}_{\mathbb{R}}=\{\{0,1\},\{\cdot+\cdot, \cdot \times \cdot\},\{\cdot<\cdot\}\}$ with the field structure of $\mathbb{R}$ as we know it. Examples of terms would be $a+b,((x+y) \times(a+b))+1$ and 1 , while examples of sentences would be $a=b$ and $a \times t=t+1$. As you can see, using only these symbols, it makes no difference if we say that it has the structure of $\mathbb{R}$ or $\mathbb{N}$. That's because, once again, structure is not language's domain. If two languages have the same symbols, it's only expected that a valid term or sentences in one is also valid in the other.

Example. Let's take $\mathcal{L}=\{\cdot \in \cdot\}$ the language used in set theory. Yes, it only uses one symbol, I know it's strange at first. Since we know the symbol $\in$, is it functional or relational? One can think that without function and constant symbols, terms can still exist as terms of complexity 1 as variable symbols while sentences cannot exist without a relational symbol. But it's not actually true, given that we said earlier that $=$ is common to all languages. Still, this symbol is indeed a relational symbol, since we know beforehand that things like $x \in \mathbb{R}$ and $\pi \in \mathbb{N}$ make sense on their own, so they must be sentences. Such language has only two types of sentences then: $a \in b$ and $a=b$.

Example. So far, we only gave examples of languages already in use on other areas of mathematics. Let's take a more abstract one, with no meaning to us whatsoever:

$$
\mathcal{L}=\{\{\dagger, \diamond, \star\},\{\mapsto \cdot \subset \cdot / \cdot / \cdot \supset\},\{\bowtie \cdot, \cdot \sqcap \cdot\}\}
$$

Terms written in $\mathcal{L}$ include things like $\mapsto(\mapsto(\diamond))$ and $\subset x / \mapsto y /(\subset x / \dagger / \dagger \supset) \supset$. Sentences would look something like $\bowtie \mapsto \subset \star / \mapsto \subset t / \dagger / \mapsto t \supset / \mapsto(\subset \star / \diamond / x \supset) \supset$. You see, we can barely tell if those are really terms and sentences at a glance, we have to put quite an effort to do so. Even when we do know what they are, we can't tell nothing more about each string of these symbols. That's the point we're trying to make. All of the previous terms and propositions should feel like these: meaningless.

That last example was way too radical. Let's make one last example, another abstract one but one in which we feel more comfortable and familiar with. 
Example. Take $\mathcal{L}=\{\alpha, \beta, f(\cdot, \cdot), g(\cdot), \cdot R \cdot, \cdot S \cdot\}$. Sentences would be like:

$$
\begin{gathered}
f(x, y)=f(y, g(x)) ; \\
g(g(x)) R g(x) ; \\
f(\alpha, \alpha)=f(\beta, \beta) ; \\
f(g(x), g(y)) S f(x, y) .
\end{gathered}
$$

That feels a lot better. Even looks like real mathematics! But since we don’t know what $\alpha$ and $\beta$ are and neither have defined $f, g, R$ and $S$, none of this means anything to us. We can see that these things make sense, but we know nothing about them. It all goes to show the power of notation and familiarity. A powerful notation helps a lot.

\section{A.1.2 Formulas}

Here is when we start with logic systems. A logic system is, in a certain point of view, some kind of sublanguage common to all other languages. This new language has symbols that will form more complex phrases that we will call formulas. Just as sentences contain terms, formulas will contain sentences. Formulas will be our most important type phrases, and they are the building blocks of what we call mathematics. They are mathematical logic's main object of study.

We have two types of new symbols we want to introduce: connectives and quantifiers.

\begin{tabular}{|c|c|}
\hline \multicolumn{2}{|c|}{ Connectives } \\
\hline Symbol & Name \\
\hline$\neg$ & not \\
$\vee$ & and \\
$\longrightarrow$ & or \\
$\longleftrightarrow$ & implies \\
\hline
\end{tabular}

\begin{tabular}{|c|l|}
\hline \multicolumn{2}{|c|}{ Quantifiers } \\
\hline Symbol & Name \\
\hline$\exists$ & exists \\
$\forall$ & for all \\
\hline
\end{tabular}


We are going to explain the connectives first. In a certain sense, they are akin to functional symbols, but instead of taking terms and outputting terms, connectives take formulas and output formulas. The negation is a unary symbol while the rest are binary symbols. For the quantifiers, they are their own type of symbol. For now, let's just say that they accept variables (variables, not other types of terms) and one formula to form another formula. With that information in mind, we can properly define formulas the same way we defined terms.

Definition A.1.4. Let $\mathcal{L}$ be a language. Given $\varphi$ a finite sequence of $\mathcal{L}$-symbols, connectives and quantifiers, we say that $\varphi$ is a formula if:

- $\varphi$ is a sentence, in this case, we say that it is also a atomic formula, or a formula with complexity 1 ;

- Given $\alpha$ and $\beta$ other formulas such that the their maximum complexity is $n$, we say $\varphi$ is a compound formula of complexity $n+1$ if either $\varphi=\exists x(\alpha), \varphi=\forall x(\alpha), \varphi=\neg \alpha$, $\varphi=\alpha \wedge \beta, \varphi=\alpha \vee \beta, \varphi=\alpha \longrightarrow \beta$ or $\varphi=\alpha \longleftrightarrow \beta$;

This is our main object of study: formulas. They will be our propositions, premises, conclusion, affirmations and even theorems and axioms. They are the ultimate phrases and will build all of mathematics. A theory is essentially a set of formulas, and a proof is a sequence of formulas. But as of yet, we still didn't gave them any meaning. For now, they are still only a structure we use to write symbols. Before doing that, we are going to talk a little more about variables, conventions and notation.

So, the quantifier symbols might have bugged you a bit for the way they work on the language. They are the only "operator" that takes different types of inputs and write them on an unusual way. Actually, since you already know what they are going to mean in the future, you already know why they should work like this, but even then they have a linguistic impact on the formulas. Let's take a more practical example to explain it. Let's take this formula, on the language $\mathcal{L}=\{0,1,+, \times\}$ :

$$
\varphi=\forall x(\exists y(x+y=0))
$$

This is a very familiar formula, one of the formulas that defines what a field is. It is saying that every $x$ has a $y$ such that $x+y=0$. You see, on this formula, we use two symbols of variables but they are not actually variables on the sense that we got used to. The variables that we are used to can be swapped for anything on their domain, and that's 
what defines variables in mathematics: they are placeholders. But on this case, we can't swap them for something else. An analogy on computer coding would be to think that the usual variables are variables that the user inputs a value for them when running the program while these variables in $\varphi$ are variables we define on the code to help the program run, where the user never actually sees their value or is even aware of their existence. In our case, what binds our variables to our code, our formula? The quantifiers.

Let's change $\varphi$ a little bit so we can see more clearly the message that I'm trying to convey. Consider this formula:

$$
\varphi_{0}=\exists y(x+y=0) .
$$

Now it makes sense for us to ask if this formula has a "real" variable or not. We can clearly see that $x$ is the variable in this case. Going back to our previous knowledge on algebra and giving back the meaning to these symbols, we know that we can judge if $\varphi_{0}$ is true or not depending on the $x$ and the universe we are in. If we are in $\mathbb{R}, \varphi_{0}$ is true for all $x$. If we are in $\mathbb{N}$, it is only true for $x=0$. It is very different than $\varphi$, where we can judge it's truthfulness right away.

Definition A.1.5. Given a language $\mathcal{L}$ and an $\mathcal{L}$-formula $\varphi$, we say that a variable $x$ appearing in $\varphi$ is bound to $\varphi$ if it's first appearance is right after a quantifier. Otherwise, it is a free variable.

You see, the discussion that led us to this definition was all about interpreting the formulas and their quantifiers but this definition is totally free of these concepts. Bound and free variables are a linguistic concept. We could have defined it right after we defined formulas, but this discussion is important to understand this definition. We keep going back to this discussion about interpreting formulas and sentences because we rely on the interpretation to guide us, to let us know what are we doing, to let us know what question are we trying to answer. But logic is meant to exist in a vacuum. It does exist in a vacuum, it was built to do so and thus it can be understood in isolation, but doing so is probably the worst way to go. That's why we keep going back to these discussions. We already know where we want to arrive and how we want to walk, so let's not forget.

Definition A.1.6. Given a language $\mathcal{L}$ and an $\mathcal{L}$-formula $\varphi$, we say that $\varphi$ is an open formula if it has free variables. Otherwise, we say that it is a closed or formal formula.

You see, the whole point of quantifiers is to actually bind variables to the formula that precedes them. We usually call this formula the scope of the bound variable and it's 
quantifier. We will see later that a quantifier that binds a variable that does not appear in it's scope is useless. We should also take care not to write variables on the start of a formula and bind it later to an inner formula. Doing so is not entirely wrong, but it creates confusion because it means that we are using the same symbol to two different objects.

To end this discussion about formulas, let's introduce notation conventions that make our writing more edible. Let's fix a language $\mathcal{L}$. If we have an $\mathcal{L}$-formula $\varphi$ in which the variables $x_{1}, \cdots, x_{n}$ are free, we will write it like this:

$$
\varphi\left(x_{1}, \cdots, x_{n}\right)
$$

If it starts with consecutive quantifiers, we can eliminate the parenthesis in a certain manner. Let's give an example:

$$
\forall x(\forall y(\exists z(\varphi(x, y, z))))=\forall x \forall y \exists z(\varphi(x, y, z))=(\forall x \forall y \exists z)(\varphi(x, y, z)) .
$$

You might find other variations among these lines in other books and papers. I think what's really important is that you stick to the same pattern to avoid confusion. Also notice how we only bound variables to formulas in which they were previously free. That's because of what we talked about some paragraphs earlier. If you always bind variables to formulas in which they were free before and take care not to compose it later to other formulas in which the same variable symbol appears later, be they free or bound, it will cause problems.

We are ready for the next part of mathematical logic, the part where we finally give meaning to these symbols. But first, let's give one last classification for formulas.

Definition A.1.7. Given a language $\mathcal{L}$ and an $\mathcal{L}$-formula $\varphi$, we say that it is an existential formula if it is of the form $\varphi=\exists x(\psi(x))$ and that it is an universal formula if it is of the form $\varphi=\forall x(\psi(x))$, where in both cases $\psi(x)$ is some $\mathcal{L}$-formula with a free variable $x$.

\section{A.2 Logics}

Now that we have an alphabet and a structure on how to write words with it, we need a structure to make sense out of our words. We are finally going to give meaning to our symbols and make them work like we have always used them. This what a logic does. 
So, let's give the symbols the meaning we made them to have. To do so, we need to discuss what a theory is, and it will also give us the idea of what a proof is, which takes us to theorems. Let's start with a discussion, as always.

What is a theory? What we expect to find when we see this word? Usually it's a whole new area of study, with it's set axioms as a starting point and a bunch of theorems derived from the axioms and previous theorems. The logical view of theories should be no different. With what we learned so far, we would need a language and a set of formulas to be our axioms.

But first, we need to develop a logic system. A system in which we write sentences, interpret them and conclude things out of them. You know, when we write things like "X implies $\mathrm{Y}$ " and "X is true" and then we conclude "Y is true", we are using logic. That's the concept we want to formalize here, for what is a theory if not a bunch of premises that we use to conclude new premises to later use to discover new premises?

\section{A.2.1 Propositional logic}

To make a logic system, we need to interpret the conjunctions, quantifiers and the equal symbols. The rest depends on language, so they should be abstracted here. From now on, let's assume that every formula we write is of some fixed language $\mathcal{L}$.

Now, let's think about how we want those symbols to work, starting from the conjunctions. So, when we write $\alpha \wedge \beta$, we expect it to mean "if $\alpha$ and $\beta$ are both true, then $\alpha \wedge \beta$ is also true". What about $\alpha \longrightarrow \beta$ ? It should be "if both $\alpha$ and $\alpha \longrightarrow \beta$ are true, then $\beta$ is also true". You see, their intention is to give us new formulas if we have other formulas already. In other words, we are inferring an affirmation out of other affirmations. Isn't that how we prove things in mathematics? That's why connectives and quantifiers are very important for mathematics. With them, we can form the inferring rules which we need to make proofs.

Definition A.2.1. An inferring rule is a rule that lets us conclude a formula out of other formulas. The starting formulas are called the rule's premises, and the formula we get out of it is it's conclusion. If the premises are $P_{1}, \cdots, P_{n}$ and the conclusion is $C$, we write:

$$
\frac{P_{1}, \cdots, P_{n}}{C}
$$

So, now that we have ways to go from one phrase to another, we have a way to go 
from our axioms to our theorems. Let's take $\Gamma$ a set of $\mathcal{L}$-formulas and some inferring rules. If we put only true formulas in $\Gamma$ and use rules that only yield true conclusions out of true statements, then we have a way to make proofs. That is, we take what we know and conclude new things with it by using the rules of inferring, that means we can finally give proofs a formal definition.

Definition A.2.2. Given a set $\Gamma$ of $\mathcal{L}$-formulas and some $\mathcal{L}$-formula $\varphi$, a proof of $\varphi$ in $\Gamma$ is a finite sequence $\left(p_{1}, \cdots, p_{n}\right)$ of $\mathcal{L}$-formulas such that $p_{n}=\varphi$ and, for each $i=1, \cdots, n, p_{i}$ is either in $\Gamma$ or is a conclusion from an inferring rule derived from formulas in $\left\{p_{1}, \cdots, p_{i-1}\right\}$. If there is a proof for $\varphi$ in $\Gamma$, we say that $\Gamma$ deduces $\varphi$ and we write $\Gamma \vdash \varphi$.

Now we know what a proof is, and we haven't even formally defined truth yet. Can we even do it, though? Philosophically speaking, the concept of truth is discussed even today. Mathematically, every definition is some proposition we say is true about whatever we are trying to define. If we don't know what truth is, how can we say something is true to define truth in the first place? So, if we can't define truth, does it mean that nothing is truly true? But if nothing is true, does that mean everything is false? But we also need to know what truth is to know what falsehood is. It all seems like a trap, a vortex from which we cannot escape with common reason. Luckily, we are mathematicians. None of this matters to us. We just give names to ideas so we can refer to them easier later on, even though these names make no sense at all with this idea. Sometimes we even give the same name to totally different, unrelated ideas. Other times we give more than one name to the same idea. So let's just name something "truth" and call it a day.

Definition A.2.3. Given a set $\Gamma$ of $\mathcal{L}$-formulas, we say that some $\mathcal{L}$-formula $\varphi$ is true in $\Gamma$ if and only if $\Gamma \vdash \varphi$. We say that $\varphi$ is false in $\Gamma$ if and only if $\Gamma \vdash \neg \varphi$.

Most books don't define truth this way. Usually, they go with truth tables and functions, assigning a truth value for each atomic formula and a table for each connective that allows us to conclude if any formula is true or false based on the atomic formulas that compose it. I prefer this definition because this is how we actually think on the other areas of mathematics. Also, by this definition we can't claim every formula is either true or false, which is how things are, not only in the rest of mathematics but also in the real life too. The bad part about it is that it might happen that some formula is both true and false at the same time, depending on $\Gamma$. We will talk about these things in more detail later, but this "problem" is not actually a problem. It's not because this definition is not really important. As said before, it's just a name given to this idea, and there is nothing wrong with a set $\Gamma$ deducing both $\varphi$ and $\neg \varphi$. It sounds weird to say something is true and false at the same time, but we are used to say that something is open and closed at the same time 
too. Differently than topology though, we won't use any $\Gamma$ that says some $\varphi$ is both true and false. Again, more on that later.

Ok, enough digressions. The first system we are going to learn is called propositional logic. You see, there are many ways to axiomatize it. Most of them, and the most famous and used indeed, only use the symbols $\longrightarrow$ and $\neg$ because they are enough to get the job done. The good part about the truth tables is that they are a very effective tool to find equivalent expressions. If the reader is familiar with them, they can easily check that $\longrightarrow$ and $\neg$ can write formulas that have the same truth values than $\wedge, \vee$ and $\longleftrightarrow$. If they are not familiar with truth tables, we can simply define those symbols as expressions using only $\longrightarrow$ and $\neg$. That's what we are going to do.

\section{Propositional Logic}

\section{Language:}

$$
\mathcal{L}=\{\neg, \longrightarrow\}
$$

\section{Axioms:}

1. $\alpha \longrightarrow(\beta \longrightarrow \alpha)$

2. $(\alpha \longrightarrow(\beta \longrightarrow \gamma)) \longrightarrow((\alpha \longrightarrow \beta) \longrightarrow(\alpha \longrightarrow \gamma))$

3. $(\neg \alpha \longrightarrow \neg \beta) \longrightarrow((\neg \alpha \longrightarrow \beta) \longrightarrow \alpha)$

\section{Inferring rules:}

$$
\frac{\alpha, \alpha \longrightarrow \beta}{\beta} \quad \mathrm{MP}, \text { "modus ponens" }
$$

In this table, $\alpha, \beta$ and $\gamma$ are variables for formulas. That is, these axioms are true for all formulas in their place. Together with the connectives, these axioms and modus ponens, they form what we call first order logic, but for now we are going to focus on propositional logic.

The inferring rule combined with these axioms are enough to characterize the symbols $\neg$ and $\longrightarrow$ as we know them. Not only for them, but they also help us define the missing symbols. Let's define them. 


$$
\begin{aligned}
\alpha \wedge \beta: & \neg(\alpha \longrightarrow \neg \beta) \\
\alpha \vee \beta: & \neg \alpha \longrightarrow \beta \\
\alpha \longleftrightarrow \beta: & (\alpha \longrightarrow \beta) \wedge(\beta \longrightarrow \alpha)
\end{aligned}
$$

We made these symbols and these axioms and rules so that our symbols behave like we want them to behave, that is, $\alpha \longrightarrow \beta$ meaning "is $\alpha$ is true, then $\beta$ is true" and the other symbols behaving similarly to how we already use them. How do we know they act like this? By demonstrating these basic properties.

So, not only to show that this axiomatization is good, but to also show examples of proofs and how they work, we are going to prove some basic logic facts that we expect these symbols to satisfy.

Example. $\vdash \alpha \longrightarrow \alpha$. When we write $\vdash \varphi$ we mean that $\varphi$ is deduced by the axiomatization of propositional logic alone.

$$
\begin{array}{ll|l}
\text { 1. } & \alpha \longrightarrow((\alpha \longrightarrow \alpha) \longrightarrow \alpha) & \text { Ax. } 1 \\
\text { 2. } & (\alpha \rightarrow((\alpha \rightarrow \alpha) \rightarrow \alpha)) \rightarrow((\alpha \rightarrow(\alpha \rightarrow \alpha)) \rightarrow(\alpha \rightarrow \alpha)) & \text { Ax. } 2 \\
\text { 3. } & (\alpha \longrightarrow(\alpha \longrightarrow \alpha)) \longrightarrow(\alpha \longrightarrow \alpha) & \text { MP 1, 2 } \\
\text { 4. } & \alpha \longrightarrow(\alpha \longrightarrow \alpha) & \text { Ax. 1 } \\
\text { 5. } & \alpha \longrightarrow \alpha & \text { MP 4, 3 }
\end{array}
$$

We are going to prove our first important result in propositional logic. This result will make deducing new formulas far easier.

Theorem A.2.4 (Deduction Theorem). Given a language $\mathcal{L}$, a set $\Sigma$ of $\mathcal{L}$-formulas and two formulas $\varphi$ and $\psi$, then $\Sigma \cup\{\varphi\} \vdash \psi$ if and only if $\Sigma \vdash \varphi \longrightarrow \psi$.

Proof. The converse is easy, if $\Sigma \vdash \varphi \longrightarrow \psi$, then a proof of $\varphi \longrightarrow \psi$ in $\Sigma$ is also a proof in $\Sigma \cup\{\varphi\}$ and, with modus ponens, can be extended into a proof of $\psi$. We need only to do the other way. Let's say $\left(\psi_{1}, \cdots, \psi_{n}\right)$ is a formal proof of $\psi$, with $\psi_{n}=\psi$. Let's prove that $\Sigma \vdash \varphi \longrightarrow \psi_{n}$ by induction on $n$.

For the case $k=1$, we know that $\psi_{k}$ is either an axiom, belongs to $\Sigma$ or $\varphi$ because there are not two or more other formulas for it to be a MP out of. If it's an axiom or belongs to $\Sigma$, we have the following deduction of $\varphi \longrightarrow \psi_{1}$ in $\Sigma$ :
1. $\psi_{1}$ Hyp.
2. $\psi_{1} \longrightarrow\left(\varphi \longrightarrow \psi_{1}\right)$ Ax. 2
3. $\varphi \longrightarrow \psi_{1}$ MP, (1) and (2) 
In the case $\psi_{1}=\varphi$, we need to prove that $\Sigma \vdash \varphi \longrightarrow \varphi$, and we already done that on the examples of before.

Now, let's suppose $\Sigma \vdash \varphi \longrightarrow \psi_{i}$ for all $i<k$. In this case, $\psi_{k}$ can be either an axiom, in $\Sigma, \varphi$ or MP of lines $j<k$ and $l<k$. If it's an axiom, belongs to $\Sigma$ or $\varphi$, we can proceed like we did on case $k=1$. For the last case, we know that $\Sigma \vdash \varphi \longrightarrow \psi_{j}$ and $\Sigma \vdash \varphi \longrightarrow \psi_{l}$ by induction hypothesis, so there are deductions $\left(\psi_{j, 1}, \cdots, \psi_{j, m_{j}}=\varphi \longrightarrow \psi_{j}\right)$ and $\left(\psi_{l, 1}, \cdots, \psi_{l, m_{l}}=\varphi \longrightarrow\left(\psi_{j} \longrightarrow \psi_{k}\right)\right.$ in $\Sigma$, which allows us to do the following:

$$
\begin{array}{rl|l}
1 . & \psi_{j, 1} & \text { Hyp. } \\
& \vdots & \\
m_{j}-1 . & \psi_{j, m_{j}-1} & \text { Hyp. } \\
m_{j} . & \varphi \longrightarrow \psi_{j} & \text { Hyp. } \\
m_{j}+1 . & \psi_{l, 1} & \text { Hyp. } \\
& \vdots & \\
m_{j}+m_{l}-1 . & \psi_{l, m_{l}-1} & \\
m_{j}+m_{l} . & \varphi \longrightarrow\left(\psi_{j} \longrightarrow \psi_{k}\right) & \text { Hyp. } \\
m_{j}+m_{l}+1 . & \left(\varphi \longrightarrow\left(\psi_{j} \longrightarrow \psi_{k}\right)\right) \longrightarrow\left(\left(\varphi \longrightarrow \psi_{j}\right) \longrightarrow\left(\varphi \longrightarrow \psi_{k}\right)\right) & \text { Hyp. } \\
m_{j}+m_{l}+2 . & \left(\varphi \longrightarrow \psi_{j}\right) \longrightarrow\left(\varphi \longrightarrow \psi_{k}\right) & \text { Ax. } \\
m_{j}+m_{l}+3 . & \varphi \longrightarrow \psi_{k} & \text { MP. } \\
&
\end{array}
$$

This theorem is probably the most important in all propositional logic. This theorem will allow us to prove the rest of the properties of all other connectives. Notice that this theorem in conjunction with modus ponens are the main properties we expect from $\longrightarrow$. This result only shows how we are on the right track to standardizing mathematic symbols formally. Let's do more proofs now that we have this powerful tool.

Corollary A.2.5. The transitivity properties are true:

(i) $\alpha \longrightarrow \beta, \beta \longrightarrow \gamma \vdash \alpha \longrightarrow \gamma$;

(ii) $\alpha \longrightarrow(\beta \longrightarrow \gamma), \beta \vdash \alpha \longrightarrow \gamma$.

Proof. By the deduction theorem, we just need to prove that $\alpha \longrightarrow \beta, \beta \longrightarrow \gamma, \alpha \vdash \gamma$ and that $\alpha \longrightarrow(\beta \longrightarrow \gamma), \beta, \alpha \vdash \gamma$. Both are very easy. 
\begin{tabular}{ll|l} 
1. & $\alpha$ \\
2. & $\alpha \longrightarrow \beta$ & Hyp. \\
3. & $\beta$ \\
4. & $\beta \longrightarrow \gamma$. \\
5. & $\gamma$ & MP. \\
Hyp. & MP.
\end{tabular}

\begin{tabular}{ll|l} 
1. & $\alpha$ \\
2. & $\alpha \longrightarrow(\beta \longrightarrow \gamma)$ & Hyp. \\
3. & $\beta \longrightarrow \gamma$ & Hyp. \\
4. & $\beta$ & MP. \\
5. & $\gamma$ & Hyp. \\
MP.
\end{tabular}

Proposition A.2.6. The following properties are true:

(i) $\vdash \neg \neg \alpha \longrightarrow \alpha$;

(ii) $\vdash \alpha \longrightarrow \neg \neg \alpha$.

Proof. For the first one:
1. $(\neg \alpha \longrightarrow \neg \neg \alpha) \longrightarrow((\neg \alpha \longrightarrow \neg \alpha) \longrightarrow \alpha)$
Ax. 3
2. $\neg \alpha \longrightarrow \neg \alpha$
Example
3. $(\neg \alpha \longrightarrow \neg \neg \alpha) \longrightarrow \alpha$
transitivity 1,2
4. $\neg \neg \alpha \longrightarrow(\neg \alpha \longrightarrow \neg \neg \alpha)$
Ax. 1
5. $\neg \neg \alpha \longrightarrow \alpha$

For the second one:
1. $(\neg \neg \neg \alpha \longrightarrow \neg \alpha) \longrightarrow((\neg \neg \neg \alpha \longrightarrow \alpha) \longrightarrow \neg \neg \alpha)$ Ax. 3
2. $\neg \neg \neg \alpha \longrightarrow \neg \alpha$
First part
3. $(\neg \neg \neg \alpha \longrightarrow \alpha) \longrightarrow \neg \neg \alpha$
MP 1, 2
4. $\alpha \longrightarrow(\neg \neg \neg \alpha \longrightarrow \alpha)$
Ax. 1
5. $\alpha \longrightarrow \neg \neg \alpha$
transitivity 3,4

Proposition A.2.7. $\vdash \neg \alpha \longrightarrow(\alpha \longrightarrow \beta)$.

Proof. By the deduction theorem, we will prove $\neg \alpha, \alpha \vdash \beta$ instead. 


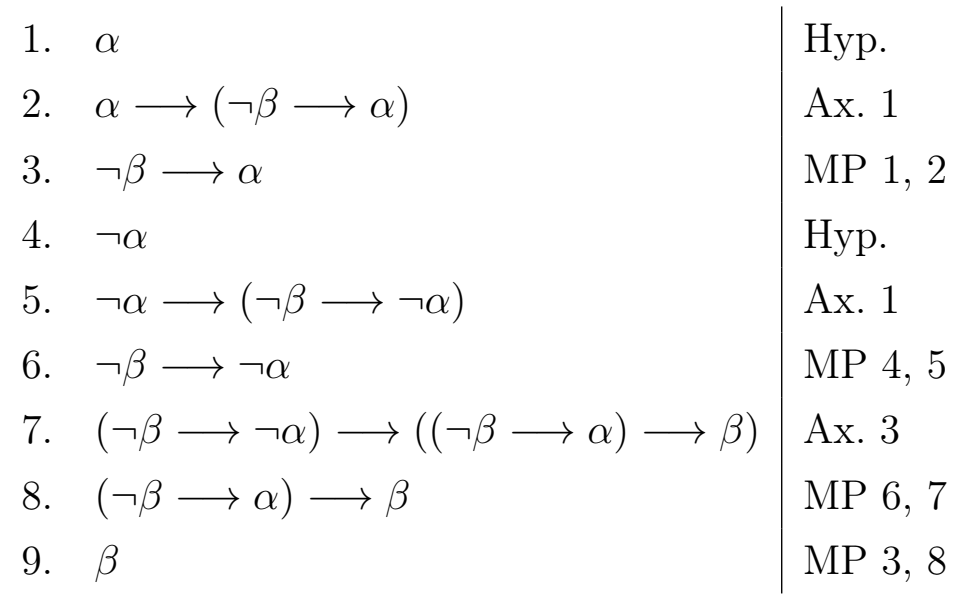

Let's stop to reflect upon the meaning of this result. We are saying that if, in a given theory, both $\alpha$ and $\neg \alpha$ are true, then all other formulas written are also true in that theory. That's why we won't be using any theories that allow such thing.

You see, by the interpretation of $\neg$, if a theory says that both $\alpha$ and $\neg \alpha$ are true it is actually contradicting itself. No theory should be contradictory, and this result proves why. In the end, both A.2.6 and A.2.7 are what characterize $\neg$ as we wanted, with the former being about the principle of the third excluded and the latter being about contradictions. We will go back to this idea later.

Proposition A.2.8. The contrapositive properties are true:

(i) $(\neg \beta \longrightarrow \neg \alpha) \longrightarrow(\alpha \longrightarrow \beta)$;

(ii) $(\alpha \longrightarrow \beta) \longrightarrow(\neg \beta \longrightarrow \neg \alpha)$.

Proof. For (i), the deduction theorem means we can prove $\neg \beta \longrightarrow \neg \alpha, \alpha \vdash \beta$ instead.

\begin{tabular}{ll|l} 
1. & $\neg \beta \longrightarrow \neg \alpha$ \\
2. & $(\neg \beta \longrightarrow \neg \alpha) \longrightarrow((\neg \beta \longrightarrow \alpha) \longrightarrow \beta)$ & Hyp. \\
3. & $(\neg \beta \longrightarrow \alpha) \longrightarrow \beta$ & Ax. 3 \\
4. & $\alpha \longrightarrow(\neg \beta \longrightarrow \alpha)$ & MP 1, 2 \\
5. & $\alpha$ & Ax. 1 \\
6. & $\neg \beta \longrightarrow \alpha$ & Hyp. \\
7. & $\beta$ & $\operatorname{MP~4,~5~}$ \\
& $\operatorname{MP~3,6}$
\end{tabular}

For (ii), analogously, we need to prove $\alpha \longrightarrow \beta, \neg \beta \vdash \neg \alpha$. 


\begin{tabular}{ll|l} 
1. & $\alpha \longrightarrow \beta$ \\
2. & $\neg \neg \alpha \longrightarrow \alpha$ & Hyp. \\
3. & $\neg \neg \alpha \longrightarrow \beta$ & Prop. A.2.6 \\
4. & $\beta \longrightarrow \neg \neg \beta$ & transitiviy 1, 2 \\
5. & $\neg \neg \alpha \longrightarrow \neg \neg \beta$ & Prop. A.2.6 \\
6. & $(\neg \neg \alpha \longrightarrow \neg \neg \beta) \longrightarrow(\neg \beta \longrightarrow \neg \alpha)$ & First part \\
7. & $\neg \beta \longrightarrow \neg \alpha$ & transitivity 3, 4 \\
8. & $\neg \beta$ & MP 5, 6 \\
9. & $\neg \alpha$ & Hyp. \\
& MP 7, 8
\end{tabular}

Proposition A.2.9. The conjunction rule is true:

$$
\vdash \alpha \longrightarrow(\beta \longrightarrow \alpha \wedge \beta) \text {, also can be written as } \frac{\alpha, \beta}{\alpha \wedge \beta}
$$

Proof. You see, by the deduction theorem, proving this formula is equivalent to proving $\alpha, \beta \vdash \alpha \wedge \beta$, which is exactly what that rule-like notation would mean. First, consider the following:

$$
\begin{aligned}
& \alpha, \alpha \longrightarrow \neg \beta \vdash \neg \beta: \\
& \text { 1. } \alpha \quad \text { Hyp. } \\
& \text { 2. } \alpha \longrightarrow \neg \beta \quad \text { Hyp. } \\
& \begin{array}{l|l}
\text { 3. } \neg \beta & \text { MP 1, } 2
\end{array}
\end{aligned}
$$

By two uses of the deduction theorem, we get that $\vdash \alpha \longrightarrow((\alpha \longrightarrow \neg \beta) \longrightarrow \neg \beta)$. Now, from the contrapositive, we get

$$
\vdash((\alpha \longrightarrow \neg \beta) \longrightarrow \neg \beta) \longrightarrow(\neg \neg \beta \longrightarrow \neg(\alpha \longrightarrow \neg \beta)),
$$

which by transitivity with the first formula, nets us

$$
\vdash \alpha \longrightarrow(\neg \neg \beta \longrightarrow \neg(\alpha \longrightarrow \neg \beta))
$$

which by deduction two times means $\alpha, \neg \neg \beta \vdash \neg(\alpha \longrightarrow \neg \beta)$, which can finally be rewritten as $\alpha, \neg \neg \beta \vdash \alpha \wedge \beta$. However, deduction and A.2.6 gives us $\beta \vdash \neg \neg \beta$, which means $\alpha, \beta \vdash \neg \neg \beta$ and thus, a deduction of $\neg \neg \beta$ can be continued into a deduction of $\alpha \wedge \beta$ since all it needs is $\alpha$ and $\neg \neg \beta$ as hypothesis, which finally gives us $\alpha, \beta \vdash \alpha \wedge \beta$.

Proposition A.2.10. The inverses of the conjunction rule are also true, that is,

$$
\frac{\alpha \wedge \beta}{\alpha} \quad \text { and } \quad \frac{\alpha \wedge \beta}{\beta}
$$


Proof. We want to prove that $\neg(\alpha \longrightarrow \neg \beta) \vdash \alpha$ and $\neg(\alpha \longrightarrow \neg \beta) \vdash \beta$. Using the deduction theorem, we can prove instead $\vdash \neg(\alpha \longrightarrow \neg \beta) \longrightarrow \alpha$ and $\vdash \neg(\alpha \longrightarrow \neg \beta) \longrightarrow \beta$.
1. $(\neg \alpha \longrightarrow(\alpha \longrightarrow \neg \beta)) \longrightarrow(\neg(\alpha \longrightarrow \neg \beta) \longrightarrow \alpha)$
2. $\neg \alpha \longrightarrow(\alpha \longrightarrow \neg \beta)$
Contrapositive
3. $\neg(\alpha \longrightarrow \neg \beta) \longrightarrow \alpha$
A. 2.7
MP 1, 2
1. $(\neg \beta \longrightarrow(\alpha \longrightarrow \neg \beta)) \longrightarrow(\neg(\alpha \longrightarrow \neg \beta) \longrightarrow \beta)$
2. $\neg \beta \longrightarrow(\alpha \longrightarrow \neg \beta)$
Contrapositive
3. $\neg(\alpha \longrightarrow \neg \beta) \longrightarrow \beta$
Ax. 1
MP 1,2

These rules are what define $\wedge$ as we know it. Assuming them let us also trivially characterize $\longleftrightarrow$ too, as follows.

Corollary A.2.11. The following rules are true:

(i) $\alpha \longrightarrow \beta, \beta \longrightarrow \alpha \vdash \alpha \longleftrightarrow \beta$;

(ii) $\alpha \longleftrightarrow \beta \vdash \alpha \longrightarrow \beta$;

(iii) $\alpha \longleftrightarrow \beta \vdash \beta \longrightarrow \alpha$.

These also allow us to simplify the contrapositive and A.2.6 on the following way:

(iv) $\alpha \longleftrightarrow \neg \neg \alpha$;

(v) $(\alpha \longrightarrow \beta) \longleftrightarrow(\neg \beta \longrightarrow \neg \alpha)$.

Now, we need to show that $\vee$ works as intended. But actually, we already did.

Proposition A.2.12. The disjunction rules are true, which are:

$$
\frac{\alpha}{\alpha \vee \beta} \quad \text { and } \quad \frac{\beta}{\alpha \vee \beta} \text {. }
$$

Proof. The first reads as $\alpha \vdash \neg \alpha \longrightarrow \beta$, which is $\alpha, \neg \alpha \vdash \beta$, which we proved in A.2.7. The other reads as $\beta \vdash \neg \alpha \longrightarrow \beta$, which is $\vdash \beta \longrightarrow(\neg \alpha \longrightarrow \beta)$, which is the first axiom.

We are going to enunciate some other basic properties that can be deduced with these techniques shown here, but we won't prove them. The interested reader can refer to [Men09], the book where most of these proofs came from. 
Proposition A.2.13. The following properties are true:

(i) $\vdash \alpha \wedge \beta \longleftrightarrow \beta \wedge \alpha$;

(ii) $\vdash \alpha \wedge(\beta \wedge \gamma) \longleftrightarrow(\alpha \wedge \beta) \wedge \gamma$;

(iii) $\vdash \alpha \vee \beta \longleftrightarrow \beta \vee \alpha$;

(iv) $\vdash \alpha \vee(\beta \vee \gamma) \longleftrightarrow(\alpha \vee \beta) \vee \gamma$

(v) $\vdash \alpha \wedge(\beta \vee \gamma) \longleftrightarrow(\alpha \wedge \beta) \vee(\alpha \vee \gamma)$;

(vi) $\vdash \alpha \vee(\beta \wedge \gamma) \longleftrightarrow(\alpha \vee \beta) \wedge(\alpha \vee \gamma)$;

(vii) $\vdash \neg(\alpha \wedge \beta) \longleftrightarrow(\neg \alpha \vee \neg \beta)$;

(viii) $\vdash \neg(\alpha \vee \beta) \longleftrightarrow(\neg \alpha \wedge \neg \beta)$;

(ix) $\vdash \neg(\alpha \longrightarrow \beta) \longleftrightarrow(\alpha \wedge \neg \beta)$.

Of all these properties, the first four allow us some conventions to eliminate parenthesis. They are:

$$
\alpha \wedge(\beta \wedge \gamma)=\alpha \wedge \beta \wedge \gamma \quad \text { and } \quad \alpha \vee(\beta \vee \gamma)=\alpha \vee \beta \vee \gamma
$$

\section{A.2.2 First order logic}

It is time we formalize the last three symbols we presented so far. Quantifiers are different because they talk about variables in the formulas, something we never have quite touched yet. Equality is not different. Up to now, the symbol $\longleftrightarrow$ has been doing the job of equality for us, but it is not equality.

You see, it's hard to explain the concept of variables in the context of logic systems because we interpret them as a placeholder for a very specific set of things, and in logic systems this set of things does not exists. It is a thing that exists only after you try to interpret the theory into something more palpable. Like developing group theory without examples of actual groups. You can show that a formula like $(\exists n>1)(\forall g \in G)\left(g^{n}=g\right)$ is valid in some groups like $\mathbb{Z}_{k}$ and not in others like $\mathbb{Z}$ because you know what the variables can become in those examples.

In our case, we don't have such examples. We are trying to make sure the language makes sense before we can apply it. That's why have to be able to abstract. Once again, 
since we know the interpretation of these symbols, we know what results we want to obtain, so the idea is to work around how to get such results.

This next logic system is called first order logic, and it is completing propositional logic. We will use all it's symbols, axioms and rules while also adding new axioms and a new inferring rule. On this table, $\varphi, \psi$ and $\chi$ are first order formulas, and when we want to highlight a specific free variable, we write it in between parenthesis after the formula, just like our convention set before. When we do that, we will also assume this variable won't appear outside it's scope. We will also use $t$ for any term written in whatever language we are applying first order logic to.

\section{First Order Logic}

\section{Language:}

$$
\mathcal{L}=\{\neg, \longrightarrow, \wedge, \vee, \longleftrightarrow \forall, \exists,=\}
$$

\section{Axioms:}

1. $\varphi \longrightarrow(\psi \longrightarrow \varphi)$

2. $(\varphi \longrightarrow(\psi \longrightarrow \chi)) \longrightarrow((\varphi \longrightarrow \psi) \longrightarrow(\varphi \longrightarrow \chi))$

3. $(\neg \varphi \longrightarrow \neg \psi) \longrightarrow((\neg \varphi \longrightarrow \psi) \longrightarrow \varphi)$

4. $(\forall x)(\varphi(x)) \longrightarrow \varphi(t)$

5. $(\forall x)(\varphi \longrightarrow \psi(x)) \longrightarrow(\varphi \longrightarrow(\forall x)(\psi(x)))$

6. $(\forall x)(x=x)$

7. $x=y \longrightarrow(\varphi(x) \longrightarrow \varphi(y))$

\section{Inferring rules:}

$\frac{\varphi, \varphi \longrightarrow \psi}{\psi} \quad \mathrm{MP}$, "modus ponens"

$\frac{\varphi(x)}{(\forall x)(\varphi(x))} \quad$ Gen, "generalization rule"

\section{Definitions:}

$$
\begin{array}{rll}
\varphi \wedge \psi: & \neg(\varphi \longrightarrow \neg \psi) \\
\varphi \vee \psi: & \neg \varphi \longrightarrow \psi \\
\varphi \longleftrightarrow \psi: & (\varphi \longrightarrow \psi) \wedge(\psi \longrightarrow \varphi) \\
(\exists x)(\varphi(x)): & \neg(\forall x)(\neg \varphi(x))
\end{array}
$$


From the connective axioms, we proved the deduction theorem for the cases where there was no generalization rule. Luckily, fixing it is easy. If $\psi_{k}$ is Gen in $\psi(x)$, then by inductive hypothesis, we know that $\vdash \varphi \longrightarrow \psi(x)$. By Gen, we get $\vdash(\forall x)(\varphi \longrightarrow \psi(x))$. By Ax. 5 and MP, we get $\vdash \varphi \longrightarrow(\forall x)(\psi(x))$, which is what we wanted to prove. So we still have the deduction theorem.

The other theorems didn't ever need any sort of argument where we supposed a step in a proof was only the old possibilities, so everything we proved last is still valid.

Let's start including the new symbols into our understanding.

Proposition A.2.14 (Existential rule). Given a language $\mathcal{L}$ and a set of $\mathcal{L}$-formulas $\Sigma$ such that $\Sigma \vdash \varphi(t)$ for a fixed $\mathcal{L}$-formula $\varphi$ and a fixed $\mathcal{L}$-term $t$ such that $x$ is free in, then $\Sigma \vdash(\exists x)(\varphi(x))$. In other words,

$$
\frac{\varphi(t)}{(\exists x)(\varphi(x))}
$$

Proof. First, we need a lemma: $\vdash(\alpha \longrightarrow \neg \beta) \longrightarrow(\beta \longrightarrow \neg \alpha)$. This can easily be proved with the contrapositive, A.2.6 and the deduction theorem in a similar manner on how was done with the conjunction rule. Now notice that what we have to prove can be rewritten as $\varphi(t) \vdash \neg(\forall x)(\neg \varphi(x))$ by definition of $\exists$.
1. $(\forall x)(\neg \varphi(x)) \longrightarrow \neg \varphi(t)$
Ax. 4
2. $((\forall x)(\neg \varphi(x)) \longrightarrow \neg \varphi(t)) \longrightarrow(\varphi(t) \longrightarrow \neg(\forall x)(\neg \varphi(x)))$
Lemma
3. $\varphi(t) \longrightarrow \neg(\forall x)(\neg \varphi(x))$
MP 1, 2
4. $\varphi(t)$
Hyp.
5. $\neg(\forall x)(\neg \varphi(x))$
MP 3, 4

Proposition A.2.15 (Particularization rule). The inverse of the generalization rule is true, that is, for any given $t$ where $x$ is free, then

$$
\frac{(\forall x)(\varphi(x))}{\varphi(t)} .
$$

Proof.
1. $(\forall x)(\varphi(x)) \longrightarrow \varphi(t) \mid \mathrm{Ax} .4$
2. $(\forall x)(\varphi(x)) \quad$ Hyp.
3. $\varphi(t)$ MP 1, 2

Corollary A.2.16. $(\forall x)(\varphi(x)) \vdash \varphi(x)$. 
These two rules are how we always used those quantifiers. Not much mystery to these rules. It all proceeds like it did on the connectives section. Using the results from there and a bit of trial and error, one can prove all the rest of these properties, so we will omit those proofs. Again, one can find some of these in [Men09], where most of those deductions came from.

Proposition A.2.17. The following properties are true:

(i) $(\forall x)(\varphi(x)) \longrightarrow(\exists x)(\varphi(x))$;

(ii) $(\forall x)(\varphi(x) \longleftrightarrow \psi(x)) \longrightarrow((\forall x)(\varphi(x)) \longleftrightarrow(\forall x)(\psi(x)))$;

(iii) $(\forall x)(\forall y)(\varphi(x, y)) \longleftrightarrow(\forall y)(\forall x)(\varphi(x, y))$;

(iv) $\neg(\forall x)(\varphi(x)) \longleftrightarrow(\exists x)(\neg \varphi(x))$;

(v) $\neg(\exists x)(\varphi(x)) \longleftrightarrow(\forall x)(\neg \varphi(x))$.

We only need to interpret $=$ now. Keep in mind that some sources do not consider $=$ to be a symbol of first order logic. So the $=$ symbol is a part of the language using first order logic and the two last axioms are included in the theories using it. Here, we will include it in first order because it makes our lives easier. If a theory does not need to use equality, it can simply not use the symbol and the axioms.

Proposition A.2.18. Given any three terms $t, s$ and $r$, the following properties are true:

(i) $\vdash t=t$ (reflexive);

(ii) $t=s \vdash s=t$ (symmetric);

(iii) $t=s, s=r \vdash t=r$ (transitive).

Proof.

$\vdash t=t$

1. $(\forall x)(x=x) \mid$ Ax. 6

2. $t=t \quad$ Particularization, 1 


\begin{tabular}{|c|c|}
\hline \multicolumn{2}{|l|}{$t=s \vdash s=t$} \\
\hline 1. $\quad t=s \longrightarrow(t=t \longrightarrow s=t)$ & Ax. 7 \\
\hline 2. $t=s$ & Hyp. \\
\hline 3. $\quad t=t \longrightarrow s=t$ & MP 1,2 \\
\hline 4. $t=t$ & Prop. (i) \\
\hline 5. $s=t$ & MP 3, 4 \\
\hline \multicolumn{2}{|l|}{$t=s, s=r \vdash t=r$} \\
\hline 1. $t=s$ & Hyp. \\
\hline 2. $s=t$ & Prop. (ii) \\
\hline 3. $s=t \longrightarrow(s=r \longrightarrow t=r)$ & Ax. 7 \\
\hline 4. $\quad s=r \longrightarrow t=r$ & $\mathrm{MP} 1,4$ \\
\hline 5. $s=r$ & Hyp. \\
\hline 6. $t=r$ & $\operatorname{MP} 4,5$ \\
\hline
\end{tabular}

And this is the one main property that equality has that we know: it's an equivalence relation. After all those deductions, we are finally ready to say that our mathematical language and theory are ideal. All the symbols we use have the properties we wanted. With all the formalism obtained, we can probably delve deeper into the theory of first order logic to find deeper properties about mathematical theories. Of course, it was already done by mathematicians before. And the findings changed mathematics as we knew it.

\section{A.3 Theories}

So far, we talked a lot about theories but only referred to them formally as "sets of formulas of a given language". That was mostly because more than that was no use for us back then, and also, theories are exactly that: a set of formulas of a given language plus the symbols, axioms and inferring rules of some logic system. To put it simply, one can build different logics. By logic I mean the symbols of connectives, quantifiers and equality together with the axioms and inferring rules.

While there are other logic systems beyond propositional logic and first order logic, we are going to focus only in first order logic for this part. Not only it is the most important of logics, but it is the one that we have the most interesting results that later led to the creation of more complex and expressive logic systems. 
Definition A.3.1. Let $\mathcal{L}$ be a language. $A$ first order $\mathcal{L}$-theory is a set $\Sigma$ of $\mathcal{L}$-formulas together with the first order logic.

Of course, there are other types of logics beside first order logic being used in mathematics, but right now we will focus on first order logic because, not only we know it, but it's also the most important one. We are saying this because not all theories in mathematics are first order. We will talk more about such theories later, on the next session.

Now, let's give some examples of first order theories to let us know which ones can be properly axiomatized in first order logic.

\section{A.3.1 Initial concepts}

Example. Group Theory

\section{Language:}

$$
\mathcal{L}=\{e, \cdot\}
$$

\section{Axioms:}

1. $(\forall x)(\forall y)(\forall z)((x \cdot y) \cdot z=x \cdot(y \cdot z))$

2. $(\forall x)(x \cdot e=e)$

3. $(\forall x)(\exists y)(x \cdot y=e)$

This is the easiest example of theory we studied at some point.

Example. Linear Order

\section{Language:}

$$
\mathcal{L}=\{<\}
$$

\section{Axioms:}

1. $(\forall x)(\neg(x<x))$

2. $(\forall x)(\forall y)(x<y \vee x=y \vee y<x)$

3. $(\forall x)(\forall y)(\forall z)(x<y \wedge y<z \longrightarrow x<z)$

Another example we know but not as a theory. Different examples would include $\mathbb{N}$ as a linear order that is also a well order, $\mathbb{R}$ as an example of dense order and $[0,1]$ as a dense order that has maximal and minimal points.

Example. Peano's Axioms 


\section{Language:}

$$
\mathcal{L}=\{0,+, \cdot, s\}
$$

\section{Axioms:}

$$
\begin{aligned}
& \text { S1. }(\forall x)(\neg 0=s(x)) \\
& \text { S2. }(\forall x)(\forall y)(s(x)=s(y) \longrightarrow x=y) \\
& \text { A1. }(\forall x)(x+0=0) \\
& \text { A2. }(\forall x)(\forall y)(x+s(y)=s(x+y)) \\
& \text { M1. }(\forall x)(x \cdot 0=0) \\
& M 2 . \quad(\forall x)(\forall y)(x \cdot s(y)=x \cdot y+x) \\
& I(\varphi(x)) . \quad(\varphi(x) \wedge(\varphi(x) \longrightarrow \varphi(s(x)))) \longrightarrow(\forall x)(\varphi(x))
\end{aligned}
$$

This is an example of an infinite theory. The last axiom is written in this way because it's actually one axiom for each formula $\varphi(x)$. If you remember a different version of Peano's axioms, one that did not include + and ', that's because you've seen the non-first order version. This is one theory that has a more complete version outside of first order logic. You see, we showed propositional logic and first order logic, but there are other logic systems beyond these two. Just as first order logic completes propositional logic, second order logic completes first order logic. There are many other types of logic, but for this work, we will only present and work with first order logic.

Before we begin the theory, let's introduce some new notation.

Definition A.3.2. Given $\mathcal{L}$ a language and $\Sigma$ a first order $\mathcal{L}$-theory, we say that $\mathcal{L}$ is $\Sigma$ 's signature, and we denote it by $\mathcal{L}=\operatorname{sig}(\Sigma)$. A formula $\varphi$ such that $\Sigma \vdash \varphi$ is called a $\Sigma$-theorem. We denote the set of all $\Sigma$-theorems by $\operatorname{Th}(\Sigma)$.

With these, we can talk about theories and theorems without first mentioning the language in which they are written in.

Let's recall what we said earlier about A.2.7. Let $\Sigma$ be first order theory. If there is a formula $\varphi$ for which both $\varphi$ and $\neg \varphi$ are theorems, then it means that everything is also a theorem in $\Sigma$. All notion of truth loses it's sense in $\Sigma$. Earlier than that, we said that we won't be using theories that allow a sentence to be true and false at the same time. We know why now. If there is a sentence that is both true and false, than every sentence is also true and false. Such theory is completely useless.

Definition A.3.3. Given $\Sigma$ a first order theory, we say that it is consistent if it has no theorem $\varphi$ such that $\neg \varphi$ is also a theorem. Otherwise, we say that it is inconsistent. 
When a theory is inconsistent, it means that it's axioms are contradicting each other somehow, directly or indirectly. This means that we can't take any set of formulas and call it a theory, one must take care not to assume too many things and end up contradicting itself. How do we know if a theory is inconsistent? Is there a way to check all formulas?

Definition A.3.4. Given $\Sigma$ a first order theory, we say that it is complete if for every formula $\varphi, \varphi$ is a theorem or $\neg \varphi$ is a theorem.

An ideal theory would be one that is complete and consistent. Unfortunately, it's impossible to take every possible formula and either prove or disprove it because there are infinite many formulas. Also, is it possible for a theory not to be complete? This means that in such a theory there would be affirmations that are impossible to prove or disprove.

Consider the group theory $\Sigma$, for instance. Since we have examples of non-abelian groups, then it must mean that $\Sigma$ can't prove $(\forall x)(\forall y)(x \cdot y=y \cdot x)$. On the other hand, there are also examples of abelian groups, therefore it can't prove it's negation too!

Definition A.3.5. Given a $\Sigma$ a first order theory, we say that a formula $\varphi$ is independent of $\Sigma$ is neither $\varphi$ nor $\neg \varphi$ are theorems. We also say a set $\left\{\varphi_{1}, \cdots, \varphi_{n}\right\}$ of formulas is independent if each formula can't be proven nor disproven by the others.

When we make a theory, we need axioms to have a ground so we can build structures on it. So usually the axioms are independent. But of course, it may not be the case as we start out and redundancy might happen. But is it a bad thing? If we have a redundant axiom, nothing bad will happen since it is already a theorem, so even if it weren't an axiom we could still use it to prove other things. At best, it could make proving things easier or having less things to prove, which are all good things. At worst, it could make our theory less elegant.

\section{A.3.2 Results}

Theorem A.3.6 (Finiteness Theorem). Given $\Sigma$ a first order theory and $\varphi$ such that $\Sigma \vdash \varphi$, then there exists a finite $\Sigma_{0} \subset \Sigma$ such that $\Sigma_{0} \vdash \varphi$.

Proof. Given that every deduction has only finite many steps, then there can be only finite many axioms in $\varphi$ 's proof.

Theorem A.3.7 (Compactness Theorem). Given $\Sigma$ a first order theory, then $\Sigma$ is consistent if and only if all finite parts $\Sigma_{0} \subset \Sigma$ are consistent. 
Proof. If $\Sigma$ is consistent, let's suppose a finite $\Sigma_{0} \subset \Sigma$ is inconsistent. Taking a formula $\varphi$ such that $\Sigma_{0} \vdash \varphi$ and $\Sigma_{0} \vdash \neg \varphi$. Because $\Sigma_{0} \subset \Sigma$, proofs of these formulas in $\Sigma_{0}$ are also proofs in $\Sigma$, and so it would mean that $\Sigma$ is inconsistent.

For the converse, let's suppose $\Sigma$ is inconsistent while all it's finite parts are not. Take a formula $\varphi$ such that $\Sigma \vdash \varphi$ and $\Sigma \vdash \neg \varphi$. By finiteness, there are finite parts $\Sigma_{1} \subset \Sigma$ and $\Sigma_{2} \subset \Sigma$ such that $\Sigma_{1} \vdash \varphi$ and $\Sigma_{2} \vdash \neg \varphi$ which, in a similar fashion to the last case, would mean the finite part $\Sigma_{1} \cup \Sigma_{2}$ is inconsistent.

These results may be simple to prove, but they are very powerful tools. The compactness theorem might as well be considered our first great result.

Lemma A.3.8. Given $\Sigma$ a consistent first order theory and a formula $\varphi$ independent of $\Sigma$, then both $\Sigma \cup\{\varphi\}$ and $\Sigma \cup\{\neg \varphi\}$ are consistent.

Proof. Let's suppose $\Sigma \cup\{\varphi\}$ is inconsistent. By compactness, let's take a finite $\Sigma_{0} \subset \Sigma \cup\{\varphi\}$ that is inconsistent. Since $\Sigma$ is consistent, $\Sigma_{0}$ cannot be a part of it, which implies that $\varphi \in \Sigma_{0}$. Again, by $\Sigma$ 's consistency, $\Sigma_{1}=\Sigma_{0} \backslash\{\varphi\}$ is consistent. Because $\Sigma_{1} \cup\{\varphi\}$ is inconsistent, then

$\Sigma_{1} \cup\{\varphi\} \vdash \neg \varphi \Longrightarrow \Sigma_{1} \vdash \varphi \Longrightarrow \neg \varphi \Longrightarrow \Sigma_{1} \vdash \neg(\varphi \wedge \varphi) \Longrightarrow \Sigma_{1} \vdash \neg \varphi \vee \neg \varphi \Longrightarrow \Sigma_{1} \vdash \neg \varphi$

proves $\varphi$ is not independent of $\Sigma$. The case for $\Sigma \cup\{\neg \varphi\}$ is done in the same way.

Theorem A.3.9 (Lindenbaum). Given $\Sigma$ an incomplete and consistent first order theory, there is an extension $\bar{\Sigma} \supset \Sigma$ with the same signature that is consistent and complete.

Proof. Consider $X$ the universe of all consistent extensions of $\Sigma$ partially ordered by $\subset$. We are going to prove that all chains have an upper bound and use Zorn's lemma.

As usual, given a chain $\mathcal{C}$, it's upper bound is $\bigcup \mathcal{C}$. We need to prove it is consistent. Let's suppose it's not. By compactness, there is some inconsistent finite part $C_{0} \subset \bigcup \mathcal{C}$. Then, there is some $C \in \mathcal{C}$ such that $\Sigma \cup C_{0} \subset C \in \mathcal{C}$, which means $C$ is inconsistent.

Now we have our maximal consistent extension $\bar{\Sigma}$. Let's prove it is complete. If it wasn't, then there would be some independent formula $\varphi$ of $\bar{\Sigma}$, which means $\bar{\Sigma} \cup\{\varphi\}$ is a consistent extension of $\bar{\Sigma}$ by A.3.8.

Remark. This proof uses the Zorn's lemma, which is a tool from set theory. You can see this property in more detail in Appendix B. Although we used it in this proof, in Lindenbaum's 
first version of this theorem, he didn't need to use this property, but instead, he had to assume that $\Sigma$ 's signature was countable.

You see, with very simple concepts like consistency and completeness, one can already get to very interesting results. There are more interesting things we would like to show, but to do that we need to introduce another concept.

\section{A.4 Models}

In logic we have those three main objects of study: logics, theories and models. Theories and models are so interdependent and synchronized that it's hard to study them isolated. We will talk about the concept of satisfiability now. Most books introduce it together with the idea of deductibility, as they are somewhat similar. But first we need to understand the concept of a model.

Let's think about plane geometry. The Elements by Euclid is a very famous book in mathematics, and the first to propose mathematical theories how we know today. He presented axioms and formalized what we view as 2-dimensional geometry. And then there was this whole deal with his 5th postulate, where everybody was trying to prove it from the other postulates. With the language we just develop, they thought that the 5th postulate was dependent on the others. We know today that they are independent. How did they prove this independence? With model theory.

A model for a theory $\Sigma$ is essentially an example of a structure that satisfies the axioms of $\Sigma$. It's an interpretation of the theory. $\mathbb{R}^{2}$ is a model for euclidean geometry. The Poincaré's Disk $D$ is a model for hyperbolic geometry. $\mathbb{Z}$ is a model for group theory. $\mathbb{Q}$ can be a model for linear order or a model for field theory, depending on the interpretation we are taking.

Definition A.4.1. A structure is a quintuple $(M, \sigma, \mathcal{C}, \mathcal{F}, \mathcal{R})$ such that $M$ is any non-empty set called the structure's domain, $\sigma$ is a language called the structure's signature, $\mathcal{C}$ is a function that assigns to each constant $\sigma$-symbol $c$ an element $c^{M} \in M, \mathcal{F}$ is a function that assigns to each n-ary function $\sigma$-symbol $f$ an $n$-ary function $f^{M}: M^{n} \rightarrow M$ and $\mathcal{R}$ is a function that assigns to each n-ary relation $\sigma$-symbol $R$ an n-ary relation $R^{M} \subset M^{n}$. We simply denote $(M, \sigma, \mathcal{C}, \mathcal{F}, \mathcal{R})$ by it's domain $M$, and if it's signature is a given language $\mathcal{L}$, we may say that $M$ is an $\mathcal{L}$-structure.

These are the objects of study in model theory. They will be our models. It makes 
sense from a language perspective to call it a model for $\mathcal{L}$, but so far we can't say anything about axioms. With the definitions of $\mathcal{F}$ and $\mathcal{R}$, perhaps we can make the case for atomic formulas? I mean, we may say that for any given terms $t_{1}, \cdots, t_{n}$ and relation $R$, we can say that $M$ satisfies $R\left(t_{1}, \cdots, t_{n}\right)$ if each $t_{i}$ is of the form $f_{i}\left(c_{1, i}, \cdots, c_{k_{i}, i}\right)$ and, denoting $t_{i}^{M}=f_{i}^{M}\left(c_{1, i}^{M}, \cdots, c_{k_{i}, i}^{M}\right),\left(t_{1}^{M}, \cdots, t_{n}^{M}\right) \in R^{M}$. But what about variables? Right now, we are very limited. Well, remembering our first order logic, if our theory deduces $\varphi(x)$, then it also deduces $(\forall x)(\varphi(x))$. In addition, we have proven that $(\forall x)(\varphi(x)) \vdash \varphi(x)$, meaning actually that $(\forall x)(\varphi(x)) \longleftrightarrow \varphi(x)$ in all languages applied to first order logic. Now, given our interpretation of quantifiers, this is where they really do their purpose. Remember the discussion we had about quantifiers, variables and models when defining first order logic? It's only in model theory that they truly fulfill the role we tried to give them.

Definition A.4.2. Given an $\mathcal{L}$-structure $M$ and $\mathcal{L}$-formulas $\varphi(\bar{x})$ and $\psi(\bar{x})$, where $\bar{x}$ is the usual string of variables $x_{1}, \cdots, x_{n}$ with $n$ up to the formula where it's written, let's define satisfiability inductively in the formulas' complexity in the following way:

- If $\varphi(\bar{x})$ is the atomic formula $R\left(t_{1}(\bar{x}), \cdots, t_{k}(\bar{x})\right)$, we say that $M$ satisfies $\varphi(\bar{x})$ and write $M \models \varphi(\bar{x})$ if $\left(t_{1}^{M}\left(\bar{x}, \cdots, t_{k}^{M}(\bar{x})\right) \in R^{M}\right.$ for all $\bar{x} \in M$ and say that $M \not \models \varphi(\bar{x})$ otherwise;

- If $\varphi$ and $\psi$ have complexity less than or equal to $n$, then $M \models \varphi \longrightarrow \psi$ if and only if $M \models \psi$ or $M \not \varphi \varphi$

- If $\varphi$ has complexity less than $n$, we say that $M \models \neg \varphi$ if and only if $M \forall \varphi$;

- If $\varphi(x)$ has complexity less than $n$, we say that $M \models(\forall x)(\varphi(x))$ if and only if $M \models \varphi$.

Given a first order theory $\Sigma$, if a structure $M$ is such that $M \models \Sigma$, we say that $M$ is a model of $\Sigma$ or that $M$ models $\Sigma$.

Corollary A.4.3. Given an $\mathcal{L}$-structure $M$, then for all $\mathcal{L}$-formula $\varphi$ we either have $M \models \varphi$ or $M \models \neg \varphi$.

This is the formal definition of a model. With common sense, it is easy to see that if $\Sigma \vdash \varphi$ and $M \models \Sigma$, then $M \models \varphi$. That's what we've been witnessing our whole lives in mathematics, after all!

Proposition A.4.4. Given a first order theory $\Sigma, M$ a model for it and a formula $\varphi$ such that $\Sigma \vdash \varphi$, then $M \models \varphi$. 
Proof. By the definition of satisfiability, one can easily think that induction on $\varphi$ 's complexity is the way to go. If one thinks that, one is right.

In the case $n=1$, then $\varphi$ must be an axiom, which means $M \models \varphi$.

In the case $n=k$ where the result is true for all complexities less than $k$, if the last line is an axiom, then this axiom is $\varphi$ which means $M \models \varphi$. If the last line is MP in lines $i<k$ and $J<k$ with and $\varphi$ 's proof is $\left(\varphi_{1}, \cdots, \varphi_{k}\right)$, then the proofs $\left(\varphi_{1}, \cdots, \varphi_{i}\right)$ and $\left(\varphi_{1}, \cdots, \varphi_{j}\right)$ prove, with inductive hypothesis, that $M \models \varphi_{i}$ and $M \models \varphi_{j}$. By definition of MP, we know that, without loss of generality, $\varphi_{j}$ is actually $\varphi_{i} \longrightarrow \varphi_{k}$. By the definition of $M \models \varphi_{i} \longrightarrow \varphi_{k}$, then we must have that $M \models \varphi_{k}$.

We can sleep with clear consciences now. It is not that theorem that will kill all of mathematics. Thinking about that result, we will make the following notation:

Definition A.4.5. Given a theory $\Sigma$, if all models $M$ for it are such that $M \models \varphi$ for a given formula $\varphi$, we say that $\varphi$ is a logical consquence or a semantic consequence of $\Sigma$. We denote that property by $\Sigma \models \varphi$.

So we just proved that $\Sigma \vdash \varphi$ implies $\Sigma \models \varphi$. The most natural question after knowing this is: What about it's converse? Does $\Sigma \models \varphi$ implies $\Sigma \vdash \varphi$ ?

Theorem A.4.6 (Gödel's Completeness Theorem I). Let $\Sigma$ be a first order theory and $\varphi$ a $\operatorname{sig}(\Sigma)$-formula. Then, the following affirmations are equivalent:

(i) $\Sigma \models \varphi \Longrightarrow \Sigma \vdash \varphi$;

(ii) If $\Sigma$ is consistent, then $\Sigma$ has a model.

Proof. To see that (i) implies (ii), let's suppose (i) and that $\Sigma$ has no model. Since it has no model, $\Sigma \models \varphi$ is true for all $\varphi$ due to emptiness. This means that $\Sigma \vdash \varphi$ for all $\varphi$, which means $\Sigma$ is inconsistent. To see that (ii) implies (i), A.3.8 implies that either $\Sigma \vdash \varphi$ or $\Sigma \cup\{\neg \varphi\}$ is consistent. But it can't be the latter because all models for $\Sigma$ satisfy $\varphi$, which means that (ii) and $\Sigma \cup\{\neg \varphi\}$ 's consistency would lead to $\Sigma \cup\{\neg \varphi\}$ having a model $M$ such that $M \models \neg \varphi$ and since $M$ also models $\Sigma$, then $M \models \varphi$, which is a contradiction. So it must be that $\Sigma \vdash \varphi$.

Theorem A.4.7 (Gödel's Completeness Theorem II). Given a theory $\Sigma$, then $\Sigma$ is consistent if and only if it has a model. 
Proof. If $\Sigma$ was inconsistent and had a model $M$, then for a given formula $\varphi$, we would have that $\Sigma \vdash \varphi$ and $\Sigma \vdash \neg \varphi$ would mean that $M \models \varphi$ and $M \models \neg \varphi$ at the same time, which is impossible. So we just have to suppose that $\Sigma$ is consistent and we have to construct a model for it.

The strategy we will use to do this proof is not the original one Gödel used back in 1929. The strategy we will use was created 20 years later by Léon Henkin. It is a method to construct models that came to be very powerful. It's called Method of Constants or Henkin Method. This method requires knowledge of ordinals and cardinals of set theory. Feel free to see appendix B if you need it. So, let's get to it.

We have that $\Sigma$ is consistent. Let $\kappa$ be the cardinality of $\operatorname{sig}(\Sigma)=\mathcal{L}=(C, F, R)^{\prime}$ 's symbols. Let $D=\left\{d_{\alpha}: \alpha<\kappa\right\}$ be a set of new constant symbols. Let's take $\mathcal{L}(D)=$ $(C \cup D, F, R)$ as a new language. Then, take $\left\{\varphi_{\alpha}: \alpha<\kappa\right\}$ the set of all $\mathcal{L}(D)$-formulas. Let's build the sequence of consistent sets $\Sigma_{\alpha}$ recursively in the following way:

- $\Sigma_{0}=\Sigma$;

- Suppose we have $\Sigma_{\alpha}$ consistent for a given $\alpha<\kappa$.

- If $\Sigma_{\alpha} \cup\left\{\varphi_{\alpha}\right\}$ is inconsistent, $\Sigma_{\alpha+1}=\Sigma_{\alpha}$;

- If $\Sigma_{\alpha} \cup\left\{\varphi_{\alpha}\right\}$ is consistent and $\varphi_{\alpha}$ is not of the form $(\exists x)(\psi(x)), \Sigma_{\sigma+1}=\Sigma_{\alpha} \cup\left\{\varphi_{\alpha}\right\}$;

- If $\Sigma_{\alpha} \cup\left\{\varphi_{\alpha}\right\}$ is consistent and $\varphi_{\alpha}=(\exists x)\left(\psi_{\alpha}(x)\right)$, then let's take $\xi_{\alpha}=\min \{\xi<\kappa$ : $d_{\xi}$ does not appear in any formula in $\left.\Sigma_{\alpha}\right\}$ and make $\Sigma_{\alpha+1}=\Sigma_{\alpha} \cup\left\{\varphi_{\alpha}, \psi_{\alpha}\left(d_{\xi_{\alpha}}\right)\right\}$. It's consistent because $\Sigma_{\alpha} \cup\left\{\varphi_{\alpha}\right\}$ is consistent, which means that if $\Sigma_{\alpha+1}$ were inconsistent, then $\Sigma_{\alpha} \cup\left\{\varphi_{\alpha}\right\} \vdash \neg \psi_{\alpha}\left(d_{\xi_{\alpha}}\right)$. Because $d_{\xi_{\alpha}}$ does not appear in any formula in $\Sigma_{\alpha}$, the only way to deduce $\neg \psi_{\alpha}\left(d_{\xi_{\alpha}}\right)$ from $\Sigma_{\alpha} \cup\left\{\varphi_{\alpha}\right\}$ is by using the particularization rule, which would imply that $\Sigma_{\alpha} \cup\left\{\varphi_{\alpha}\right\} \vdash(\forall x)\left(\neg \psi_{\alpha}(x)\right)$. Because $(\forall x)\left(\neg \psi_{\alpha}(x)\right)$ is $\neg(\exists x)\left(\psi_{\alpha}(x)\right)$ (which is $\left.\neg \varphi_{\alpha}\right)$ by definition, that would mean $\Sigma_{\alpha} \cup\left\{\varphi_{\alpha}\right\}$ is inconsistent;

- Suppose that, for a limit ordinal $\alpha<\kappa$, we have $\Sigma_{\xi}$ consistent for all $\xi<\alpha$. Then we have that $\Sigma_{\alpha}=\bigcup\left\{\Sigma_{\xi}: \xi<\alpha\right\}$ is consistent because if it weren't, then by compactness there would be an inconsistent finite part $\Gamma \subset \Sigma_{\alpha}$, which means that there is $\xi<\alpha$ such that $\Gamma \subset \Sigma_{\xi}$, making $\Sigma_{\xi}$ inconsistent.

Now we have a theory $\Sigma_{\kappa}$ that is consistent and complete by construction. We are going to build a structure out of $D$ that will model $\Sigma_{\kappa}$. Consider the equivalence relation $\sim$ in $D$ defined by $d \sim d^{\prime}$ if and only if the formula $d=d^{\prime}$ is in $\Sigma_{\kappa}$. Now let's take $M=\{[d] \subset D$ : 
$d \in D\}$ the set of equivalence classes of $\sim$ and make an interpretation of it to make it a model for $\Sigma_{\kappa}$.

- For every $d \in D$, make $d^{M}=[d]$;

- For every $c \in \mathcal{L}$, the formula $(\exists x)(x=c)$ will always be consistent with any $\mathcal{L}(D)$ theory, so that means that there is some $\alpha<\kappa$ such that $\varphi_{n}=(\exists x)(x=c)$, which by construction, means $\Sigma_{\alpha+1}=\Sigma_{\alpha} \cup\left\{(\exists x)(x=c), d_{\xi_{\alpha}}=c\right\}$. So we can interpret $c^{M}=\left[d_{\xi_{\alpha}}\right]$

- For every $n$-ary function symbol $f$, if the formula $f(\bar{d})=e$ for some $\bar{d}$ and $e$ in $D$, then we can have $f^{M}(\overline{[d]})=[e]$;

- For every $n$-ary relation $R$, we can make $(\overline{[d]}) \in R^{M}$ if and only if $R(\bar{d})$ is in $\Sigma_{\kappa}$

It's easy to see that, by construction, $M \models \Sigma_{\kappa}$. Since $\Sigma \subset \Sigma_{\kappa}$, then $M \models \Sigma$ too.

That's the first big and important theorem of model theory. It means that for any first order theory we create, if we can use ZFC to build a model for it, it is consistent. A corolary of the completeness theorem is also a powerful tool in model theory belt:

Corollary A.4.8 (Compactness). Any first order theory $\Sigma$ has a model if and only if each finite part $\Sigma_{0} \subset \Sigma$ has a model.

We could go on to show more powerful results of model theory, but what we have developed so far is more than enough for anyone to be able to read this document's main work. For those who are curious, you can see more books on the subject. We recommend [CK90], [Mar06] and [EFT13]. 
APPENDIX A 


\section{Appendix B}

\section{Set Theory}

Now that we have our basic knowledge of logic, we can talk about set theory. This appendix's intention is to be less extensive than appendix A, for there is far more topics to cover in set theory. Our intention is to only give enough ground for any reader to understand this document's language, which is mostly the basics of ordinals and models of ZFC.

Of course, there is so much to be shown about set theory here. For those who are interested in more content and exercises, the book [HJ99] covers the basics of set theory in great detail. For those looking for advanced set theory, [Kun83] is highly recommended.

\section{B.1 Axioms of ZFC}

Differently than what was done on appendix A, we are not going to simply list them in a table. Doing so wouldn't be very productive, as it's usually very hard to read between the lines and understand the message they are trying to convey. So instead of doing this, we are going to present them and do a short discussion around the presented axiom.

As said before in an example, our language will be the relation symbol $\in$ alone. Our terms will always refer to sets. There is nothing other than sets. Even the elements of our sets are sets. Everything is a set. When we "build" a set $X$, what we actually mean is that $Z F C \vdash(\exists X)(x \in X \longrightarrow \varphi(x))$ for some quality $\varphi$ that defines $X$ or something along those lines. 


\section{Axiom 1: Extensionality}

$$
(\forall x)(\forall y)((\forall z)(z \in x \longleftrightarrow z \in y) \longrightarrow x=y)
$$

What this axiom is saying is that, given the sets $x$ and $y$, if $(\forall z)(z \in x \longleftrightarrow z \in y)$, then $x=y$. This is the set that links $=$ to $\in$ and define what makes a set different to each other. Proves most uniquenesses.

\section{Axiom 2: Comprehension}

This next axiom is actually one axiom for each formula $\varphi$ without $y$ as a free variable.

$$
(\forall z)(\exists y)(\forall x)(x \in y \longleftrightarrow x \in z \wedge \varphi(x))
$$

What this axiom is trying to say is that, given a set $z$ and a formula $\varphi$, there is a set $y \subset z$ with all $x$ such that $\varphi(x)$. You know when we write $\{x \in X: \varphi(x)\}$ ? We are actually using that axiom. We can prove two great things with this axiom:

Theorem B.1.1. There is an empty set.

Proof. Just use comprehension for $\varphi(x)$ being $\neg x=x$. Since no $x$ is such that $\neg x=x$ by the axioms of equality, no $x$ is such that $x \in y$.

From now on, we will refer to this set as $\varnothing$.

Theorem B.1.2. There is no set of all sets

Proof. If $(\exists z)(\forall x)(x \in z)$ held true, we could use the comprehension axiom to get a set $y$ such that $x \in y \longleftrightarrow x \in z \wedge \neg x \in x$, which is equivalent to $(\forall x)(x \in y \longleftrightarrow \neg x \in x)$. Particularizing it to $y$, we get that $y \in y \longleftrightarrow \neg y \in y$.

\section{Axiom 3: Replacement}

Like the axiom of comprehension, this one also comprises infinite many axioms, one for each formula $\varphi(x, y)$ without $Y$ as a free variable.

$\forall A \forall x\left(x \in A \rightarrow\left(\exists y\left(\left(\varphi(x, y) \wedge\left(\varphi\left(x, y^{\prime}\right) \rightarrow y=y^{\prime}\right) \rightarrow \exists Y \forall x(x \in A \rightarrow \exists y(y \in Y \rightarrow \varphi(x, y)))\right)\right)\right.\right.$ 
Feel free to ignore the formal axiom in symbols. What it actually means is: if $\varphi(x, y)$ is a function linking each $x \in A$ to a unique $y \in Y$, then $Y$ exists. In other words, given a set $A$ and a function $f$ linking each $x \in A$ to a unique $y$, there is $Y=\{y: y=f(x)\}$

Also like the axiom of comprehension, this one is very powerful too. Together with the axiom of comprehension, it lets us create any set. Most sets we write are a combination of those axioms in use.

\section{Axiom 4: Pairing}

$$
(\forall x)(\forall y)(\exists z)(\forall t)(t \in z \longleftrightarrow t=x \vee t=y)
$$

This means that for any $x$ and $y$, there is $\{x, y\}$. Particularizing $y=x$, we get that for all $x$ there is $\{x\}$. This also allows the construction of ordered pairs.

Definition B.1.3. Given any two $x$ and $y$, we denote $(x, y)=\{x,\{x, y\}\}$.

\section{Axiom 5: Reunion}

$$
(\forall \mathcal{F})(\exists z)(\forall x)(x \in z \longleftrightarrow(\exists y)(y \in \mathcal{F} \wedge x \in y))
$$

As the name suggests, this axioms is saying that there exists $\bigcup \mathcal{F}$. We can't use comprehension alone to get the reunion set because we would need a bigger $z$ to contain $y$ in it's statement. That's why we need to state the existence of the reunion as an axiom.

\section{Axiom 6: Power Set}

$$
(\forall X)(\exists z)(\forall y)(y \in z \longleftrightarrow(\forall x)(x \in y \longrightarrow x \in X))
$$

Again, as the name suggests, this axiom allows us to get $\mathcal{P}(X)$ the set of all subsets of $X$. We need this axiom because the power set has the same issues as the reunion set to be defined with comprehension and replacement alone. 


\section{Axiom 7: Infinity}

$$
(\exists z)(\varnothing \in z \wedge(\forall x)(x \in z \longrightarrow x \cup\{x\} \in z))
$$

This axiom is saying that there is a $z$ that has $\varnothing,\{\varnothing\},\{\varnothing,\{\varnothing\}\},\{\varnothing,\{\varnothing\},\{\varnothing,\{\varnothing\}\}\} \ldots$ We are actually saying that $x \cup\{x\}$ acts like a successor of $x$ and $z$ is inductive. Without this axiom we can't actually make any infinite set.

Definition B.1.4. Given a set $x$, we define it's successor as $S(x)=x \cup\{x\}$.

\section{Axiom 8: Foundation}

$$
(\forall x)((\exists y)(y \in x) \longrightarrow(\exists y)(y \in x \wedge \neg(\exists z)(z \in x \wedge z \in y)))
$$

This axiom is there so there are no cases of $x \in x$ or $x \in y \in x$ or any sort of cycle like $x_{0} \in x_{1} \in \cdots \in x_{n} \in x_{0}$. If you go picking elements of the elements of the elements of $x$, the sequence will be finite. It won't really be used by us on this document, so feel free to ignore it, but it's always good to be mindful of this axiom.

\section{Axiom 9: Choice}

$$
(\forall A)(\exists R)(R \text { well orders } A)
$$

Writing this one in formal language is way too complicated, so we opted to do this instead. We assure you that there is a first order formula that says that $R$ well orders $A$. In case the reader is not familiar with well orders:

Definition B.1.5. A linear order $<$ in a set $X$ is said to be a well order if every subset $A \subset X$ contains a minimal element. We say that two ordered sets $A$ and $B$ are isomorphic and we write $A \cong B$ if and only if there is a crescent bijective function between them.

This axiom is equivalent to Zorn's lemma:

Theorem B.1.6. (Zorn's Lemma) Given a set $X$ with a partial order $<$, the following properties are equivalent: 
(i) X has a maximal element;

(ii) Every $C \subset X$ such that $<$ total orders $C$ has an upper bound.

There are many equivalencies to the axiom of choice. We can't quite prove this lemma yet, but we don't intend to prove it. It is a very famous result with applications throughout all of mathematics, so most readers are probably already familiar with it.

\section{Constructions of ZFC}

Now, to see that this theory works, we need to build the basic tools and definitions of sets we use in the other areas of mathematics. Without any formal knowledge of set theory, every mathematician still knows and uses basic notions such as the $\epsilon$ and $\subset$ symbols and the operations of reunion, intersection and cartesian product. Let's think about these ideas and some others.

Reunion. The first idea that springs to mind is to simply apply the axiom of reunion. So, if one have two sets $A$ and $B$, we can define $A \cup B$ as $A \cup B=z$ where $z$ is the set obtained from the reunion axiom. But we can't actually apply it to two sets $A$ and $B$, we need a family $\mathcal{F}$ of sets to do so. In our case, we want it to be $\{A, B\}$. Luckily, the axiom of pairing got us covered. If we want the reunion of a family of sets instead, we don't need this last step with the pairing axiom.

Intersection. This one is easier. Given two sets $A$ and $B$, we can use comprehension for $A \cap B=\{x \in A: x \in B\}$. If we want to intersect a family $\mathcal{F}$ of sets, we can use comprehension for $\bigcap \mathcal{F}=\{x \in \bigcup \mathcal{F}:(\exists y)(y \in \mathcal{F} \wedge x \in y)\}$. Notice how we used $\bigcup \mathcal{F}$, be we don't actually need it. We could have taken any $A \in \mathcal{F}$ instead, given that $\mathcal{F}$ is not empty. But what would happen if it was? By looking at the axiom of reunion, one can see that $\mathcal{F}=\varnothing$ means $\cup \mathcal{F}=\varnothing$. Looking at the definition we gave for $\bigcap \mathcal{F}$, we can see that $\bigcap \mathcal{F} \subset \cup \mathcal{F}$ and that the formula inside is always false, meaning that $\bigcap \mathcal{F}=\varnothing$ for at least two different reasons. But of course, if one wants to exclude those cases, one can always define the reunion and intersection only for non empty families of sets.

Minus. Given two sets $A$ and $B$, we can define $A \backslash B=\{x \in A: x \notin B\}$ with the axiom of comprehension. 
Power set. We just need to apply the axiom of the power set, which exists solely for this. Also, notice how this also defines the symbol $\subset$, to which we can simply define as $A \subset B$ to mean $A \in \mathcal{P}(B)$.

Finite product. Given two sets $A$ and $B$, we want to define $A \times B$. With clever usage of the pairing axiom, we defined ordered pairs $(x, y)$ for any given $x$ and $y$ as $(x, y)=\{x,\{x, y\}$. This means that, if $x \in A$ and $y \in B$, then $(x, y) \in \mathcal{P}(A \cup \mathcal{P}(A \cup B))$, which lets us define the product as $A \times B=\{x \in \mathcal{P}(A \cup \mathcal{P}(A \cup B)):(\exists a)(\exists b)(a \in A \wedge b \in B \wedge x=(a, b))\}$. Again, note how $A \times B=\varnothing$ if and only if $A=\varnothing$ or $B=\varnothing$.

Defining the product of a family of sets is a tricky matter at this point. The easiest way to do so is by defining functions first.

Definition B.1.7. Given two sets $A$ and $B$, a function $f: A \longrightarrow B$ is a non empty subset $f \subset A \times B$ such that if $(a, x) \in f$ and $(a, y) \in f$, then $x=y$.

Arbitrary product. Given a family $\mathcal{F}$ of sets, we want to define $\prod \mathcal{F}$ to be the set of all functions $f: \mathcal{F} \longrightarrow \bigcup \mathcal{F}$ such that for all $A \in \mathcal{F}$, we have $f(A) \in A$. We can do so with the comprehension axiom with a long formula, so we will omit it.

As usual, let's analyze fringe cases. If $\mathcal{F}=\varnothing$, then there are no functions from it, which means $\prod \mathcal{F}=\varnothing$. But to arrive on those functions we've been through so many steps one can wonder if $\mathcal{F}=\varnothing$ is the only way to trivialize $\prod \mathcal{F}$. Out of the bat, it's easy to suspect that $\varnothing \in \mathcal{F}$ implies $\prod \mathcal{F}=\varnothing$, and it's also easy to verify that it's true. But are those the only cases?

Let's look closer at the definition of function. Given two non empty sets $A$ and $B$, are there any functions from $A$ to $B$ in the first place? Certainly. If $B \neq \varnothing$, then there is $c \in B$, which allows us to make $f(x)=c$ for all $x \in A$. But the functions in $\prod \mathcal{F}$ have an additional restriction on them. Given a non empty family $\mathcal{F}$ of non empty sets, is there a function $f: \mathcal{F} \longrightarrow \bigcup \mathcal{F}$ such that $f(A) \in A$ for all $A \in \mathcal{F}$ ? This seems obvious at first glance. Since each $A \in \mathcal{F}$ is non empty, we can take $x_{A} \in A$ in each $A \in \mathcal{F}$ and define $f(A)=x_{A}$, but when we try to use comprehension or some other axioms to construct such function, we hit an obstacle writing it in a formula. How can we quantify those $x_{A}$ and refer to them later? Doing so is exactly defining the function we want to!

If $\mathcal{F}$ is finite, then we need finite symbols to refer to each $x_{A}$, which allows us to simply write them all off in a single formula. But when $\mathcal{F}$ is infinite, listing a unique $x_{A} \in A$ 
for each $A \in \mathcal{F}$ is trickier. We know there must be such functions because we always assume on "normal mathematics" that the product of a non empty family of non empty sets is non empty. If we can't find those functions, we would need to fix our theory. Perhaps, we need to add another axiom that says those functions exist?

Here's the solution.

Theorem B.1.8. Given a non empty family $\mathcal{F}$ of non empty sets, there are functions from $\mathcal{F}$ to $\bigcup \mathcal{F}$ such that $f(A) \in A$ for all $A \in \mathcal{F}$.

Proof. For each $A \in \mathcal{F}$, the axiom of choice states that there is a well order $\leq_{A}$ on it. This means that there is a formula $\varphi(A, x)$ that says $x$ is the minimum element of $A$ in $\leq_{A}$ 's ordering, which lets us write $f=\{x \in \mathcal{F} \times \bigcup \mathcal{F}:(\exists y)(\exists z)(y \in \mathcal{F} \wedge z \in \cup \mathcal{F} \wedge x=\varphi(y, z))\}$, which is a function in the conditions we wanted.

That's actually a very easy resolution. In fact, one can even wonder if the axiom of choice was actually created just to solve this problem. In fact, the axiom of choice does seems odd when compared to the others, does it not? Truth is, stating that these functions do exist was actually the first form of the axiom of choice, and these functions were called choice functions, after all, a function with this property is choosing an element from each set in $\mathcal{F}$. As expected, the axiom of choice is independent from the rest of axioms. It is customary to refer to the axiom of choice simply by AC, and to call ZF the theory of all ZFC axioms except for $\mathrm{AC}$, and saying $\mathrm{ZF}+\neg \mathrm{AC}$ to mean $\mathrm{ZF}$ plus the negation of $\mathrm{AC}$.

Of course, the existence of these functions also prove that all sets can be well ordered. To do so, we need the theorem of transfinite recursion and as such, we can't prove it right now. As said before, there are many other equivalencies to the axiom of choice. The existence of choice functions and the fact that $\prod \mathcal{F} \neq \varnothing$ if $\mathcal{F}$ and it's elements are non empty are the most famous equivalencies. Other than those, there is Zorn's Lemma, that was already stated, there's Tychonoff's Theorem, there's the fact that all vector spaces have a base and many others. Today, the most common way to state the axiom of choice is the way we stated. It's easier to use, read and to apply. To end this section, let's discuss one last time about how we define sets, it's formalization and it's consequences to the rest of mathematics.

Final remarks. When we define set theory with a logic language as a first-order theory, we take away the notion that a set is a collection of things and replace it with symbols that make up a logical structure of a language. As of now, we are not dealing with "sets" anymore, even though we call it "set theory". The advent of modern logic gave us tools 
formal enough to create these structures that behave like our primitive idea of a set, but with formalism enough to let us see advanced things such as the axiom of choice and to apply logic's powerful results such as Gödel's theorems.

That's why we need this formalism. Logic and set theory first came to be after a lot of paradoxes came to light. This formalism fixes them and let us see now beyond what a set is supposed to be in our intuition. With the right logical structure and list of axioms, a set is whatever it can possibly be.

This theory for sets is the most used one today, although there are others. The ZF theory has this name due to Zermelo and Fraenkel, who worked on it from late 1900s to early 1920s. Adding the axiom of choice or some of it's equivalents yields ZFC.

\section{B.2 Ordinals and cardinals}

Ordinals can be seen as either an extension of the natural numbers or as a representation of each well order.

Definition B.2.1. We say that a set $X$ is transitive if and only if every $x \in X$ is also a subset of $X$. An ordinal is a transitive set that is well ordered by the relation $\in$.

Proposition B.2.2. Given $\alpha, \beta$ and $\gamma$ ordinals, the following statements hold true:

(i) If $x \in \alpha$, then $x$ is an ordinal and is an initial segment of $\alpha$;

(ii) If $\alpha \cong \beta$, then $\alpha=\beta$;

(iii) Either $\alpha \in \beta$ or $\beta \in \alpha$ or $\alpha=\beta$;

(iv) If $\alpha \in \beta$ and $\beta \in \gamma$, then $\alpha \in \gamma$;

(v) Given a non empty set $C$ of ordinals, then there is $\delta \in C$ such that for all $\xi \in C$, $\delta \in \xi$.

Proof.

(i): By definition, $x \subset \alpha$ means that it is well ordered by $\in$ and is the set of all it's predecessors. This will make it transitive too. 
(ii): Consider $x=\alpha \cap \beta$. It is not empty because $\varnothing$ is in all ordinals as their minimal elements due to transitivity and (i). So $x$ must be an initial segment of both, and so $x=\min (\alpha \backslash x)=\min (\beta \backslash x)$ which would mean $x \in x$ if either $\alpha \backslash x=\varnothing$ or $\beta \backslash x=\varnothing$. Because they are isomorphic, they must both be empty and thus $\alpha=x=\beta$.

(iii): Same as (ii).

(iv): Due to the transitive property of ordinals.

(v): Due to the (iii), take $\alpha \in C$ and consider $C \cap \alpha$. Since $\alpha$ is well ordered, $C \cap \alpha$ has a minimal element.

What we just proved is that the set of all ordinals is well ordered by $\in$. That is, if there is such a set.

Theorem B.2.3. There is no set of all ordinals.

Proof. If there were such a set $\Omega$, this set would be transitive and well ordered by $\in$, meaning it's an ordinal and belongs to itself, meaning $\Omega<\Omega$, which breaks the well order of $\in$ of ordinals.

On a different way, we could have said that $\Omega \in \Omega$ breaks the axiom of foundation to end this proof, but we said earlier that we won't need this axiom so we are avoiding it's use. Now we know that there is no set of all sets nor a set of all ordinals. Even so, we might refer to them as if there were to make notation easier. We will call $V$ the "set of all sets" and $O N$ the "set of all ordinals" so we can more easily say $x$ is a set by writing $x \in V$, and on the same idea, we can write $\alpha \in O N$ to mean $\alpha$ is an ordinal. We call those "sets" classes, so we say that $V$ is the class of all sets and $O N$ is the class of all ordinals. It also makes the notation easier for formulas that act like functions to be simply defined as functions on either $V$ or $O N$, more on those formulas later.

Also, as another interesting fact due to (iv), we can write $\alpha<\beta$ instead of $\alpha \in \beta$ because of $\beta$ 's ordering. That's how we will write when comparing them from now on. Now, of all the basic properties, let's see the most important one.

Theorem B.2.4. Let $A$ be a well ordered set. Then there is an ordinal $\alpha$ such that $A \cong \alpha$. 
Proof. First, let's denote the initial segments of $A$ by $I_{a}=\{x \in A: x<a\}$ for any given $a \in$ $A$. Let's take $S \subset A$ such that $S=\left\{x \in A:(\exists \alpha)\left(\alpha \in O N \longrightarrow \alpha \cong I_{x}\right)\right\}$. $S$ can't be empty because $\min A \in S$, as $I_{\min A} \cong 0$. Let's define $R=\left\{\alpha \in O N:(\exists x)\left(x \in A \longrightarrow I_{x} \cong \alpha\right)\right\}$. To define this set formally, we need the axiom of replacement in $S$, replacing each element by it's isomorphic ordinal.

Now, it's easy to see that both $S$ and $R$ are initial segments of $A$ and $O N$ respectively, the latter meaning that $S$ is actually an ordinal. To finish this proof, we will show that $S=A$. Supposing it's not, then we can take $a=\min (A \backslash S)$ and $\alpha \in O N$ such that $S \cong \alpha$. Then the set $I_{a}$ is actually congruent to $\alpha+1$, meaning that $a \in S$.

This means that ordinals represent well orders. Considering the axiom of choice, this also means that every set has a bijection into an ordinal.

Proposition B.2.5. If $\alpha$ is an ordinal, then $S(\alpha)=\alpha+1$ is an ordinal.

Proof. Self evident.

This property helps us see better how the ordinals look. Notice that $\alpha$ is greater than all it's elements, meaning that $\alpha+1$ is $\alpha$ with an added element greater than all it's original ones.

Now, let's take $\varnothing$ as an ordinal. We have that $\varnothing+1=\{\varnothing\}$. We can take it's successor, and it's successor's successor. With finite recursion, we can define these sets in a more formal way as $E_{0}=\varnothing$ and $E_{n+1}=S\left(E_{n}\right)$. On a careful look, we can see that these sets are actually the sets in the axiom of infinity. This means that, by the axiom of infinity there exists the set $\left\{E_{n}: n \in \mathbb{N}\right\}$, which we will call $\omega$.

One can also use finite induction to prove $E_{n}$ is an ordinal for all $n$ natural number, which means each $\omega_{1}$ is actually an ordinal! With another careful look, one can see that $\omega \cong \mathbb{N}$. And there you have it. We will use $\omega$ as our formal construction of the set of natural numbers. Notice that $\omega$ is the first "infinite" ordinal. I say "infinite" with quotation marks because we haven't formally defined finite or infinite sets yet.

Definition B.2.6. $A$ set $A$ is said to be infinite if and only if there is a bijection between $A$ and a proper subset of $A$. By proper subset, we mean a subset $B \subset A$ such that $B \neq A$.

One can prove each that $E_{n}$ is finite by induction on $n$, and the proof that $\omega$ is infinite is a widespread knowledge. 
Definition B.2.7. An ordinal $\alpha$ is said to be a successor ordinal if there is some ordinal $\alpha_{0}$ such that $\alpha=\alpha_{0}+1$. Otherwise, we say it is a limit ordinal.

It's easy to see that $\omega$ is a limit ordinal while the $E_{n}$ ' are not. Now, looking back to how we arrived at $\omega$, we can do the same technique of taking successors to build new sets. By taking $\omega$ 's successor, we arrive at a set with all natural numbers and an element greater than all naturals. That's $\omega+1=\{0,1,2, \cdots, \omega\}$. We can still take it's successor, arriving at $\omega+2=\{0,1,2, \cdots, \omega, \omega+1\}$. Doing it inductively, we arrive at the set $\{0,1,2, \cdots, \omega, \omega+$ $1, \omega+2, \cdots\}$, which we call $\omega+\omega$, or $\omega \cdot 2$. In the same manner, we can arrive at $\omega \cdot 2+1$, $\omega \cdot 3, \omega \cdot \omega=\omega^{2}$ and so on. In fact, we can define arithmetic operations with ordinals and make those ideas well defined.

Definition B.2.8 (Ordinal Arithmetic). Given ordinals $\alpha$ and $\beta$, we will define the following ordered sets:

(i) $\alpha+\beta$ is the ordinal isomorphic to the set $(\alpha \times\{0\}) \cup(\beta \times\{1\})$ ordered by $(\xi, i)<(\zeta, j)$ if and only if, $i<j$ or, if $i=j, \xi<\zeta$;

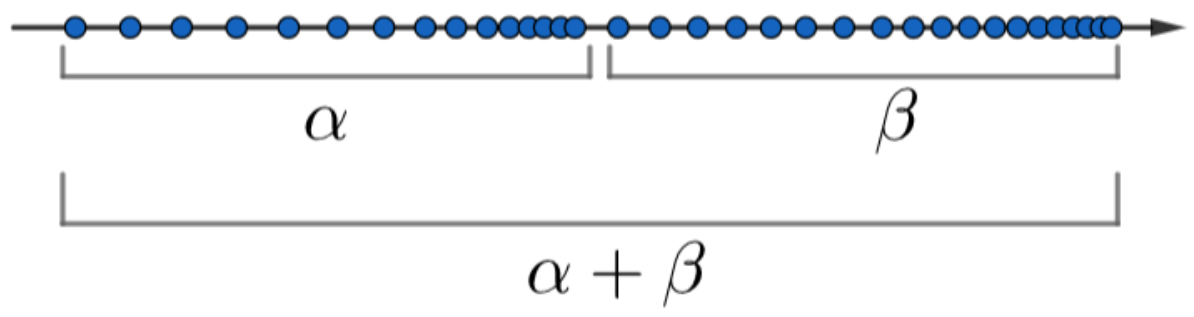

(ii) $\alpha \cdot \beta$ is the ordinal isomorphic to the set $\alpha \times \beta$ ordered by $(\xi, \zeta)<\left(\xi^{\prime}, \zeta^{\prime}\right)$ if and only if, $\zeta<\zeta^{\prime}$ or, if $\zeta=\zeta^{\prime}, \xi<\xi^{\prime}$;

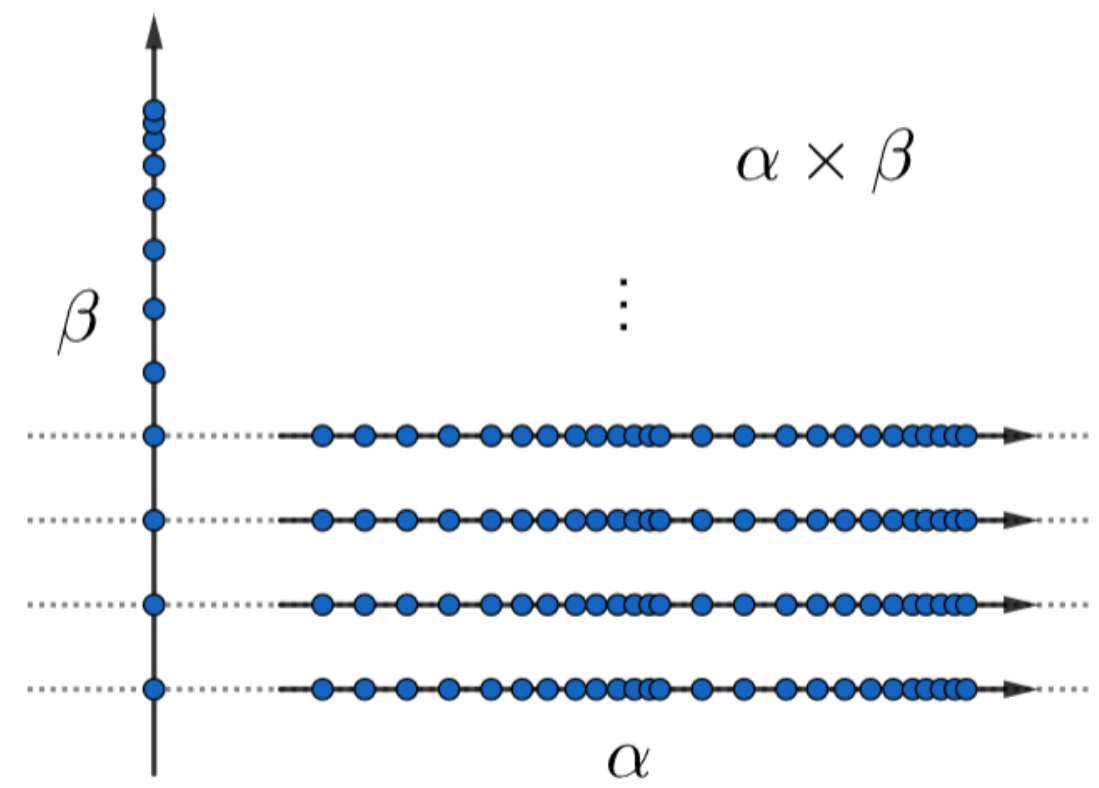


(iii) Given a set $S$ of ordinals, we define $\sup S=\bigcup S$.

There are many properties of these operations, but since those operations are not important for us, we are not going to delve deeper and prove those properties. They do not commute, though. Other than those properties, we also need to prove that they are well defined, but again, we will not do that. You can also define subtraction and division in the same way it's done in the natural numbers. In fact, it can even be proven a version of the basis representation theorem for ordinals! To do that, however, we need the transfinite induction theorem. We also need this theorem to define powers.

Transfinite induction and recursion are the main reason why we added this long section about ordinals. That and the fact that it's impossible to talk about $\omega_{1}$ without talking about ordinals first.

Theorem B.2.9 (Transfinite Induction). Let $\varphi(x)$ be a property such that if $\varphi(\beta)$ is true for all ordinals $\beta<\alpha$, then $\varphi(\alpha)$ is true. Then, $\varphi(\alpha)$ is true for all $\alpha \in O N$.

Proof. Let's suppose there is an ordinal $\alpha$ such that $\varphi(\alpha)$ is not true. Then we can define $C=\{\xi<\alpha: \neg \varphi(\xi)\}$, which should be well ordered by $\in$ and thus we can take $\delta=\min C$. This means that for all $\xi<\delta, \varphi(\xi)$ should be true, which means that $\varphi(\delta)$ is also true, which is a contradiction.

To talk about the transfinite recursion, let's make some notes about notation. Imagine a formula $\varphi(x, y)$ such that for every $x \in V$, there is a unique $y \in V$ such that $\varphi(x, y)$. There are many such formulas, such as a formula that states that $y$ is the power set of $x$ or that $y$ is the set $x \cup\{x\}$. The thing about these formulas is that they act like functions from $V$ to $V$, but since $V$ is not actually a set, we can't build a function $f: V \longrightarrow V$ such that $\varphi(x, f(x))$ for all $x \in V$. We will call those formulas class functions, as like if they were a function from a class into another. So now we can even write things like $F: O N \longrightarrow O N$ and $G: V \longrightarrow O N$ to mean something similar to the first formula on all sets.

Theorem B.2.10 (Transfinite Recursion). Given a class function $F: V \longrightarrow V$, then there is a unique class function $G: O N \longrightarrow V$ such that $G(\alpha)=F\left(G \uparrow_{\alpha}\right)$ for all $\alpha \in O N$.

Proof. Given an ordinal $\delta$, let's say that a function $g$ with domain $\delta$ is a $\delta$-approximation if it is such that $g(\xi)=F\left(g \uparrow_{\xi}\right)$ for all $\xi<\delta$. Let's see that for a given $\delta \in O N$, it's $\delta$-approximations are unique. Let's suppose $g$ and $h$ are $\delta$-approximations. If $\{\xi<\delta: g(\xi) \neq$ $\left.g^{\prime}(\xi)\right\}$ is not empty, then we can take $\gamma=\min \left\{\xi<\delta: g(\xi) \neq g^{\prime}(\xi)\right\}$. Because $\gamma$ is the 
minimal element, $g \Gamma_{\gamma}=g^{\prime} \Gamma_{\gamma}$. But that means $g(\gamma)=F\left(g \uparrow_{\gamma}\right)=F\left(g^{\prime} \Gamma_{\gamma}\right)=g^{\prime}(\gamma)$, a contradiction.

Now we will prove $G$ 's existence with by transfinite induction. Let's say that for a given ordinal $\alpha$, all $\beta<\alpha$ have $\beta$-approximations. If we prove that this means that $\alpha$ has an $\alpha$-approximation, then the transfinite induction theorem gives us that all $\alpha \in O N$ have $\alpha$-approximations, which ends our proof.

So, let's suppose all $\beta<\alpha$ have $\beta$-approximations. We know that they are all unique, so if $\beta<\beta^{\prime}<\alpha$ and $g_{\beta}$ is a $\beta$-approximation while $g_{\beta^{\prime}}$ is a $\beta^{\prime}$-approximation, then $g_{\beta^{\prime}} \uparrow_{\beta}=g_{\beta}$. With the axiom of replacement on $\alpha$, we know that there is the set of all $\beta$-approximations $S=\left\{g_{\beta}\right.$ : for all $\beta<\alpha, g_{\beta}$ is a $\beta$-approximation $\}$. With that, it's easy to see that the set $g=\bigcup S \cup\{(\alpha, F(\bigcup S))\}$ is an $\alpha$-approximation.

To link this recursion theorem to the normal finite versions, you have to think of $G$ as the sequence where each point is determined by the previous points, and $F$ is the rule which you apply to all those previous points to get the one in question. What this theorem is saying is that given the rule $F$ with the starting point $a_{0}=G(0)=F(\varnothing)$, then there are points $a_{\alpha}=G(\alpha)$ for each $\alpha \in O N$ such that $G(\alpha)=F\left(G \uparrow_{\alpha}\right)$.

We are finally ready to talk about cardinals. We will start with one of the most classic results of set theory.

Theorem B.2.11 (Cantor). Given a set $X$, there are no bijections from $X$ to $\mathcal{P}(X)$.

Proof. Let's suppose there is a bijection $f$ between them. Let's take the set $S=\{x \in X$ : $x \notin f(x)\}$. Naturally, there is some $y \in X$ such that $f(y)=S$. By definition of $S$, we would have $y \in S \longleftrightarrow y \notin S$.

With this result and the axiom of choice, we can do the following discovery:

Proposition B.2.12. There is an uncountable ordinal.

Proof. Consider $\mathcal{P}(\omega)$. It is not countable due to Cantor's theorem. With the axiom of choice, there is a well order for it. As of all well ordered sets, there is an ordinal $\alpha$ isomorphic to it. This means that $\alpha$ is uncountable.

With this, one can do the following definition: 
Definition B.2.13. Let's denote by $\omega_{1}=\min \{\xi \leq \alpha: \xi>\omega$ and $\xi$ is uncountable $\}$

This will be the main object of study in this work. This ordinal has many qualities going for it. Notice that, in the same manner, one can take $\mathcal{P}\left(\omega_{1}\right)$, well order it with an ordinal $\alpha$ and define $\omega_{2}=\min \left\{\xi \leq \alpha: \xi>\omega_{1}\right.$ and there are no bijections from $\xi$ to $\left.\omega_{1}\right\}$. Can we then use transfinite recursion to define an $\omega_{\alpha}$ analogously to each $\alpha \in O N$ ? You see, this method only gives us an ordinal from a previous one, so it means we need a way to form $\omega_{\alpha}$ for $\alpha$ limit.

Definition B.2.14. With transfinite recursion, let's define an ordinal $\omega_{\alpha}$ for each $\alpha \in O N$ in the following way:

- $\omega_{0}=\omega$;

- Given $\omega_{\alpha}$ defined, then $\omega_{\alpha+1}=\min \left\{\xi \leq \gamma: \xi>\omega_{\alpha}\right.$ and there are no bijections from $\xi$ to $\left.\omega_{\alpha}\right\}$, where $\gamma$ is an ordinal isomorphic to some well order for $\mathcal{P}\left(\omega_{\alpha}\right)$;

- Given $\alpha$ limit and $\omega_{\beta}$ defined for all $\beta<\alpha$, then $\omega_{\alpha}=\sup \left\{\omega_{\beta}: \beta<\alpha\right\}$.

This is how most transfinite recursive constructions are made. You have to specify the construction for successor ordinals and for limit ones. The step for limit ordinals is what differentiate transfinite recursion from finite recursion. Of course, sometimes it is possible for a single rule to work for both successors and limits. Let's see what's special about this sequence we just defined now.

Lemma B.2.15. Given $\alpha \in O N$, if $\xi$ is such that $\omega_{\alpha} \leq \xi<\omega_{\alpha+1}$, then there are bijections from $\xi$ to $\omega_{\alpha}$ and no bijections from $\xi$ to $\omega_{\alpha+1}$. Also, for all infinite ordinals $\xi$, there exists some $\alpha \in O N$ such that $\omega_{\alpha} \leq \xi<\omega_{\alpha+1}$.

Proof. Given $\omega_{\alpha}<\xi<\omega_{\alpha+1}$ for some $\alpha \in O N$, if there were no bijection from $\xi$ to $\omega_{\alpha}$, then we would have that $\omega_{\alpha+1} \leq \xi$ by the definition of $\omega_{\alpha+1}$. If there were some bijection from $\xi$ to $\omega_{\alpha+1}$, you could compose it with one of it's bijections to $\omega_{\alpha}$ and get a bijection from $\omega_{\alpha}$ to $\omega_{\alpha+1}$, which contradicts $\omega_{\alpha+1}$ 's definition.

For the second statement, let's consider the set $S$ of all $\omega_{\beta}$ such that $\omega_{\beta} \leq \xi$. If $S$ has a maximal element $\omega_{\alpha}$, then it's obvious that $\omega_{\alpha} \leq \xi<\omega_{\alpha+1}$. If it does not, then the only way it can happen is if $S$ is of the form $\left\{\omega_{\beta}<\xi: \beta<\alpha\right\}$ for some $\alpha \in O N$ limit. Let's consider $\delta=\bigcup S$. By definition, $\delta=\omega_{\alpha}$. Due to $S$ 's definition, $\omega_{\alpha}>\xi$ because $S$ has only elements equal to or greater than itself and $\omega_{\alpha}$ can't be in $S$ or else it would have a maximal 
element. But if $\xi<\omega_{\alpha}$, then it means there is some $\beta<\alpha$ such that $\xi<\omega_{\beta}$, contradicting $S$ 's definition.

Proposition B.2.16. Given $\alpha<\beta$ ordinals, there are no bijections from $\omega_{\alpha}$ to $\omega_{\beta}$.

Proof. Let's use transfinite induction. Let's say $\varphi(\alpha)$ means there are no bijections from $\omega_{\alpha}$ to $\omega_{\alpha}^{\prime}$ for all $\alpha^{\prime}<\alpha$. Now let's suppose $\varphi(\alpha)$ holds true for all $\alpha<\beta$ for a given $\beta$.

If $\beta$ is successor, say $\beta=\gamma+1$. By construction there are no bijections from $\omega_{\beta}$ to $\omega_{\gamma}$. Let's suppose there is a bijection $f$ from $\omega_{\beta}$ to some $\omega_{\alpha}<\omega_{\gamma}$. Since $\omega_{\gamma}<\omega_{\beta}$, we can consider the image $f\left(\omega_{\gamma}\right)$. Since it is a set of ordinals, we know it is well ordered and that it has some ordinal $\delta<\omega_{\alpha}$ isomorphic to it. By the lemma above, we can get $\alpha^{\prime}<\alpha$ such that $\omega_{\alpha^{\prime}} \leq \delta<\omega_{\alpha^{\prime}+1}$ such that there is a bijection from $\omega_{\alpha^{\prime}}$ to $\delta$. But then, by composing the functions, we get a bijection from $\omega_{\gamma}$ to $\omega_{\alpha^{\prime}}$, which contradicts the inductive hypothesis.

Now, let's say $\beta$ is limit. Supposing there is some $\alpha<\beta$ such that there is a bijection from $\omega_{\alpha}$ to $\omega_{\beta}$. Because of the inductive hypothesis, there can't be another $\alpha^{\prime}<\beta$ such that there are bijections from $\omega_{\alpha^{\prime}}$ to $\omega_{\beta}$ or else there would be a bijection from $\omega_{\alpha^{\prime}}$ to $\omega_{\alpha}$, which contradicts the inductive hypothesis. Now, with this knowledge and the fact that $\beta$ is limit, we can take some $\alpha<\gamma<\beta$ such that there are no bijections from $\omega_{\gamma}$ to $\omega_{\beta}$. In this case, we are exactly in the same conditions of the case $\beta$ successor. We can use the same argument from before to finish the proof.

These last two results show what are these ordinals important for. They represent the existence of bijections between sets. If there is a bijection between two infinite ordinals $\alpha$ and $\beta$, it means there is a unique $\gamma$ such that there are bijections from $\omega_{\gamma}$ to $\alpha$ and from $\omega_{\gamma}$ to $\beta$. In this regard, $\omega_{\gamma}$ is something like their "class". For finite ordinals, it is easy to see that there never is a bijection between two distinct finite ordinals, which means they are their own class, just like the $\omega_{\alpha}$ 's. Just like ordinals represent the well order of well ordered sets, these sets we built represent all ordinals that have bijections between each other. With the axiom of choice, they can represent all sets that have bijections between one another.

Definition B.2.17. An ordinal $\kappa$ is called a cardinal if there are no bijections from $\kappa$ to $\xi$ for all $\xi<\kappa$. The class of all cardinals will be denoted by Card.

With this definition, it's trivial to see that the sequence we constructed together with the finite ordinals are all the cardinals that exist.

Definition B.2.18. Given a cardinal $\kappa$, we write $\kappa^{+}$to mean the next cardinal above it, 
that is, given $\gamma$ isomorphic to some well ordering of $\mathcal{P}(\kappa)$,

$$
\kappa^{+}=\min \{\xi \leq \gamma: \kappa<\xi \text { and there are no bijections from } \xi \text { to } \kappa\} \text {. }
$$

Given a set $A$, we say that it's cardinality is the cardinal $\kappa$ such that there are bijections from $A$ to $\kappa$, and we denote it by $|A|$.

Remark. Notice how we used AC to construct $\kappa^{+}$from a given cardinal $\kappa$. It is not necessary, though. We decided to go through this way because most of mathematics happen assuming $\mathrm{AC}$, and using it is significantly easier in this case than not. But don't think we can't make this construction in $\mathrm{ZF}$ or $\mathrm{ZF}+\neg \mathrm{AC}$.

That's all for a good introduction to cardinals. Let's talk about cardinal arithmetics.

Definition B.2.19 (Cardinal Arithmetic). Given two cardinals $\kappa$ and $\lambda$, we define:

(i) $\kappa+\lambda=\max \{\kappa, \lambda\}$;

(ii) $\kappa \cdot \lambda=\max \{\kappa, \lambda\}$;

(iii) $\kappa^{\lambda}=\mid\{f \in \mathcal{P}(\lambda \times \kappa): f$ is a function from $\lambda$ to $\kappa\} \mid$.

As expected, it can be proven that the sum $\kappa+\lambda$ seen as a sum of ordinals has cardinality $\kappa+\lambda$ seen as a sum of cardinals, and the same is true for the multiplication. For the exponentiation, these definitions end up differently, so beware. Funny thing is, with these definitions for addition and multiplication, it makes no sense for they to be studied, so we only study exponentiation. It turns out we can't know the result of any exponentiation. Take $2^{\omega}=|\mathcal{P}(\omega)|$, for example. It is consistent with ZFC to assume that $2^{\omega}=\omega_{1}$. In fact, it's consistent to assume that it takes any value whatsoever. The same is true for any cardinal $\kappa$, it is consistent to assume that $2^{\kappa}$ is equal to any cardinal greater than $\kappa$.

Definition B.2.20. We call the continuum hypothesis the statement that $2^{\omega}=\omega_{1}$, and we denote it by $\mathrm{CH}$. We call the generalized continuum hypothesis the statement that $2^{\kappa}=\kappa^{+}$ for all $\kappa \in$ Card, and we denote it by $\mathrm{GCH}$.

As customary, one can write $\mathrm{ZFC}+\mathrm{CH}$ to mean the theory of ZFC plus the continuum hypothesis, or ZFC $+\neg \mathrm{CH}$ to mean the theory of ZFC plus the negation of the continuum hypothesis. The same for $\mathrm{ZF}+\mathrm{CH}, \mathrm{ZFC}+\mathrm{GCH}, \mathrm{ZF}+\neg \mathrm{CH}$ and $\mathrm{ZFC}+\neg \mathrm{GCH}$.

Let's prove some properties of cardinals now. 
Proposition B.2.21. Given two infinite sets $A$ and $B$, then $|A \times B|=|A| \cdot|B|$.

Proof. Suppose $A$ is the biggest set, which means $|A| \cdot|B|=|A|$. It's easy to see that $|A| \leq|A \times B| \leq|A \times A|$. If we prove that $|A \times A|=|A|$, which is the same thing as proving that $|\kappa \times \kappa|=\kappa$ to all $\omega \leq \kappa \in$ Card, everything is done. We will prove it by transfinite induction. This image will show how we will order $\omega \times \omega$.

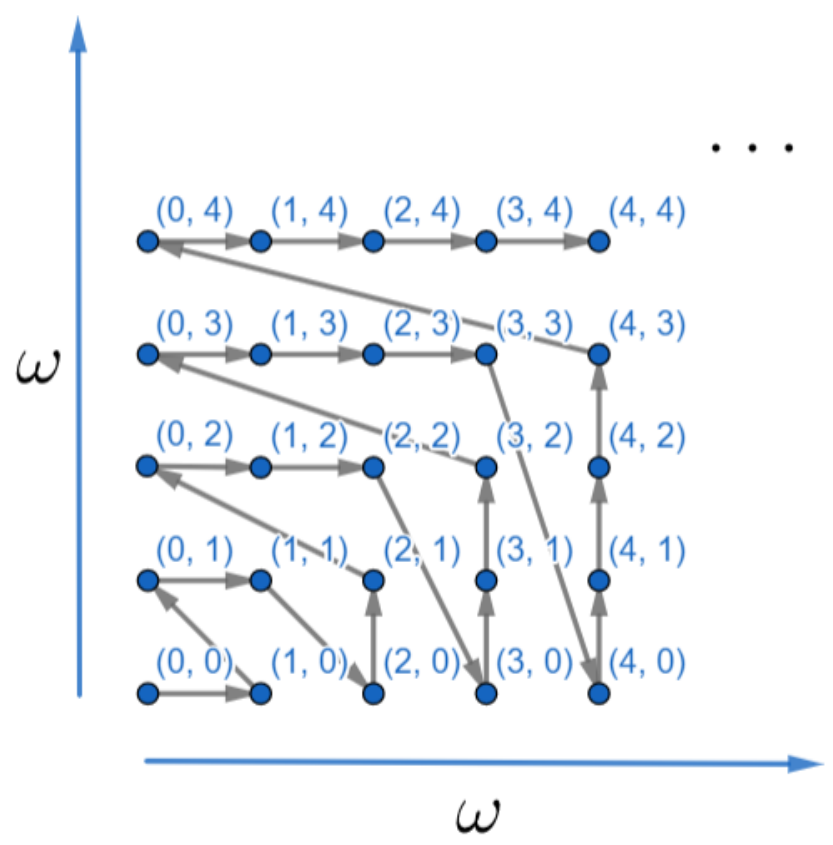

Every arrow points to the point's successor, so we have that $(0,0)<(0,1)<(1,1)<(1,0)$ and so on. It's easy to see that with this order, $\omega \times \omega$ is isomorphic to $\omega$. This scheme will well order $\alpha \times \alpha$ for every $\alpha \in O N$ in fact. Formally, it can be written as:

$$
(\xi, \zeta)<\left(\xi^{\prime}, \zeta^{\prime}\right) \Longleftrightarrow\left\{\begin{array}{l}
\max \{\xi, \zeta\}<\max \left\{\xi^{\prime}, \zeta\right\} \text { or; } \\
\max \{\xi, \zeta\}=\xi=\zeta^{\prime}=\max \left\{\xi^{\prime}, \zeta\right\} \text { or; } \\
\max \{\xi, \zeta\}=\xi=\xi^{\prime}=\max \left\{\xi^{\prime}, \zeta\right\} \text { and } \zeta<\zeta^{\prime} \text { or } \\
\max \{\xi, \zeta\}=\zeta=\zeta^{\prime}=\max \left\{\xi^{\prime}, \zeta\right\} \text { and } \xi<\xi^{\prime}
\end{array}\right.
$$

We now know that this order witnesses that $|\omega \times \omega|=\omega$. Now, let's suppose it witnesses that $|\lambda \times \lambda|=\lambda$ for all cardinals $\lambda<\kappa$ for a given $\kappa \in$ Card. If $|\kappa \times \kappa|=\kappa^{\prime}$ for some cardinal $\kappa^{\prime}>\kappa$, then we could take the initial segment $S \subset \kappa \times \kappa$ that's isomorphic to $\kappa$. Let's say that $S=\{(\xi, \zeta) \in \kappa \times \kappa:(\xi, \zeta)<(\alpha, \beta)\}$. By taking $\gamma=\max \{\alpha, \beta\}$, we know that $S \subset \gamma \times \gamma$, which means $|S| \leq|\gamma \times \gamma|$. But $\gamma<\kappa$ means $|\gamma|=\lambda<\kappa$, and so by 
inductive hypothesis, $|\gamma \times \gamma|=\lambda<\kappa$, which contradicts $|S|=\kappa$.

Proposition B.2.22. Given $\kappa \in$ Card infinite and a family of sets $\left\{X_{\alpha}: \alpha<\kappa\right\}$ such that $\left|X_{\alpha}\right| \leq \kappa$ for all $\alpha<\kappa$, then $X=\bigcup\left\{X_{\alpha}: \alpha<\kappa\right\}$ has cardinality less than or equal to $\kappa$.

Proof. It is done by comparing $X$ to $\kappa \times \kappa$. For every $\alpha<\kappa$, we can choose a one-to-one function $f_{\alpha}: X_{\alpha} \longrightarrow \kappa$. For every $x \in X$, there exists $\alpha_{x}<\kappa$ such that $x \in X_{\alpha_{x}}$, so we can define $f: X \longrightarrow \kappa \times \kappa$ such that $f(x)=\left(\alpha_{x}, f_{\alpha_{x}}(x)\right)$. We have that $f$ is one-to-one because each $f_{\alpha}$ is too. This means that $X$ has the same cardinality as $f$ 's range, which is a subset of $\kappa \times \kappa$, and so $|X| \leq \kappa$.

Corollary B.2.23. Given two infinite sets $A$ and $B$, then $|A \cup B|=|A|+|B|$.

Notice that this proposition is a more general version of the known fact that a countable reunion of countable sets is countable. With this, we can prove the following property of $\omega_{1}$ :

Proposition B.2.24. If $\left\langle\alpha_{\xi}: \xi<\omega\right\rangle$ is a crescent sequence of countable ordinals, then it does not converge to $\omega_{1}$.

Proof. Since it is crescent, they converge to $\alpha=\sup \left\{\alpha_{\xi}: \xi<\omega\right\}$. If $\alpha=\omega_{1}$, that would mean $\omega_{1}$ is a countable reunion of countable sets, making it countable.

This property of $\omega_{1}$ is very important to this work. Not only this work, but it is all over the whole set theory. One can clearly see that this is only a particular case of B.2.22, so we should be able to prove a more general version of this proposition.

Proposition B.2.25. Given $\kappa$ an infinite cardinal, if $\left\langle\alpha_{\xi}: \xi<\kappa\right\rangle$ is a crescent sequence such that $\alpha_{\xi}<\kappa^{+}$for all $\xi<\kappa$, then it does not converge to $\kappa^{+}$.

Proof. Since it converges to $\alpha=\sup \left\{\alpha_{\xi}: \xi<\kappa\right\}$, you just have to apply B.2.22.

Definition B.2.26. Given $\alpha \in O N$, it's cofinality, denoted by $\mathrm{c} f(\alpha)$, is the smallest ordinal $\beta$ such that a crescent sequence of order type $\beta$ converges to $\alpha$. Equivalently, it's the smallest ordinal $\beta$ of which a subset of $\alpha$ isomorphic to $\beta$ is unbounded in $\alpha$. If for a given $\alpha \in O N$ we have that $\operatorname{cf}(\alpha)=\alpha$, we say that $\alpha$ is regular.

It's very easy to prove that for all ordinals $\alpha, c f(\alpha)$ is a cardinal. What we just proved with the last proposition is that for all cardinals $\kappa$, it is true that $\kappa^{+}$is regular. This means that a subset of $\kappa^{+}$has cardinality $\kappa^{+}$if and only if it is unbounded in $\kappa^{+}$. This is a powerful tool that we will be using all the time in the main work of this document. 
Proposition B.2.27. Given some limit ordinal $\alpha$, then $c f\left(\omega_{\alpha}\right)=c f(\alpha)$.

Proof. Let's say that $c f(\alpha)=\delta$ and that $\left\{\alpha_{\xi}: \xi<\delta\right\}$ is a cofinal sequence in $\alpha$. Then it's easy to see that $\left\{\omega_{\alpha_{\xi}}: \xi<\delta\right\}$ is a cofinal sequence in $\omega_{\alpha}$.

If one wants to look more carefully at this last bit, the statement $c f\left(\omega_{\alpha}\right)=c f(\alpha)$ does not mean that $\omega_{\alpha}$ is not regular. That would mean that $\omega_{\alpha}=\alpha$, which is somewhat counter intuitive. Cardinals like this are called inaccessible and their existence is independent of ZFC. There are many types of different definitions and studies on inaccessible cardinals, or large cardinals, as some people like grouping these types of cardinals, but we have no need of this theory on this work.

Although there are a few more things we could speak about cardinals, we will opt not to do so, since we already covered more than enough. We have one more thing to talk about, and that is models of ZFC.

\section{B.3 Sets of hereditary cardinality}

Let's start by making a recursive construction.

Definition B.3.1. For each $\alpha \in O N$, let's define:

- $V_{0}=\varnothing$;

- $V_{\alpha+1}=\mathcal{P}\left(V_{\alpha}\right)$;

- If $\alpha$ is limit, $V_{\alpha}=\bigcup\left\{R_{\xi}: \xi<\alpha\right\}$.

Given $x \in V_{\alpha}$ for some $\alpha \in O N$, we define the rank of $\alpha$ as the ordinal $\operatorname{rank}(x)=\min \{\xi \leq$ $\left.\alpha: \xi \in V_{\xi}\right\}$.

Proposition B.3.2. For each $\alpha \in O N, V_{\alpha}$ is transitive and, if $\beta<\alpha$, then $V_{\beta} \subset V_{\alpha}$.

Proof. Transfinite induction. Supposing it's true for all $\beta<\alpha$, let's prove $V_{\alpha}$ is transitive and contains $V_{\beta}$ for all $\beta<\alpha$.

In the case $\alpha$ is limit, the definition makes it clear that $V_{\alpha}$ has all it's predecessors. To see that it's transitive, just use the fact that if $x \in V_{\alpha}$ then $x \in V_{\beta}$ for some $\beta<\alpha$, which is transitive by inductive hypothesis. 
In the case $\alpha=\beta+1$, then $V_{\alpha}=\mathcal{P}\left(V_{\beta}\right)$ is transitive because $\beta$ is transitive, and thus it also has all it's elements and therefore contains all it's predecessors.

Corollary B.3.3. Given $\alpha \in O N, V_{\alpha}$ satisfies the axiom of extensionality. In fact, the axiom of extensionality is true in any transitive set.

Proof. Given two elements $x \in V_{\alpha}$ and $y \in V_{\alpha}$, by transitiveness, $x \subset V_{\alpha}$ and $y \subset V_{\alpha}$. If $z \in x \longleftrightarrow z \in y$ is true, then they are the same subset of $V_{\alpha}$ and thus $x=y$.

This first result must paint a picture of where we want to get to. We will return to talk about formulas and satisfiability on this section.

Proposition B.3.4. Given some limit ordinal $\alpha, V_{\alpha}$ satisfies the axiom of comprehension.

Proof. The secret to the axiom of comprehension in this case lies in transitivity. When you take a set $S$ and a formula $\varphi(x)$, by construction, there is $\beta<\alpha$ such that $S \in V_{\beta}$, which means $\mathcal{P}(S) \in V_{\beta+1} \subset V_{\alpha}$. So, in this case, $\{x \in S: \varphi(x)\}$ must be in $V_{\alpha}$ too.

Next, we will talk about the axiom of foundation. Let's consider the class of all sets $X$ in $V_{\alpha}$ for some $\alpha \in O N$. We call this class $W F$. The name $W F$ means well founded sets. Those sets are built in a manner such that the relation $\in$ is well founded. We say that a relation $R$ on a set $X$ is well founded when every subset $A \subset X$ has an element $x \in A$ such that no $y \in A$ is such that $y R x$. That's the same thing of a well order, except the relation is not necessarily a linear order. This means that, by construction...

Proposition B.3.5. Given $\alpha \in O N, V_{\alpha}$ satisfies the axiom of foundation.

In fact, the axiom of foundation is equivalent to the statement $V=W F$, that is, for any given set $X$, there is $\alpha \in O N$ such that $X \in V_{\alpha}$. You see, the use of the notation $V_{\alpha}$ to get to $V=\bigcup\left\{V_{\alpha}: \alpha \in O N\right\}$ is no coincidence. This construction is due to von Neumann, and is regarded as one of the first "models" for ZFC. We talked first about the axiom of foundation before because we need $W F$ as a whole for the axiom of replacement.

Proposition B.3.6. WF satisfies the axiom of replacement.

Proof. Once again, the trick is on transitivity. Given $S \in V_{\alpha}$ and a formula $\varphi(x, y)$ on replacement's conditions, then there is some $\beta \in O N$ such that $S \in V_{\beta}$ and $y \in V_{\beta}$ for all $y$ such that $\varphi(x, y)$ for each $x \in S$. In this case, $\left\{y \in V_{\beta}:(\exists x)(x \in S \wedge \varphi(x, y))\right\}$ is an element of $V_{\beta+1}$. 
You see, since $W F$ is not something constructible with the tools we have now, we would prefer to work under some $V_{\alpha}$ instead. One solution to this problem is to accept only formulas $\varphi(x, y)$ such that there is some $\beta<\alpha$ that knows all images $y$ such that $\varphi(x, y)$ for all $x \in S$, where $S$ is the "domain" we are leaving from. That's what we are going to do.

Proposition B.3.7. WF satisfies the axiom of the power set.

Proof. Given some set $X \in W F$, there is some $\alpha \in O N$ such that $X \in R(\alpha)$, which means $\mathcal{P}(X) \in V_{\alpha+1}$.

This axiom is the hardest one to fit into some $V_{\alpha}$. The solution we will adopt is to limit the scope of sets from which we can consider the power set.

Proposition B.3.8. Given a limit ordinal $\alpha, V_{\alpha}$ satisfies the axiom of pairing.

Proof. Given two sets $X$ and $Y$ in $V_{\alpha}$, there is $\beta<\alpha$ such that $X \in V_{\beta}$ and $Y \in V_{\beta}$, which means $\{X, Y\} \in V_{\beta+1} \subset V_{\alpha}$.

Proposition B.3.9. Given a limit ordinal $\alpha, V_{\alpha}$ satisfies the axiom of reunion.

Proof. Given a set $S \in V_{\alpha}$, there is $\beta<\alpha$ such that $S \in V_{\beta}$ and, by transitivity, $S \subset V_{\beta}$. This means that $V_{\beta+1}$ has $\bigcup S$.

Proposition B.3.10. Given an ordinal $\alpha>\omega, V_{\alpha}$ satisfies the axiom of infinity.

Proof. By construction, $V_{\alpha}$ possesses all ordinals $\beta<\alpha$, which includes $\omega$.

Proposition B.3.11. Given a limit ordinal $\alpha, V_{\alpha}$ satisfies the axiom of choice.

Proof. Given a non empty family $\mathcal{F}$ of non empty sets, there is $\beta<\alpha$ such that $\mathcal{F} \in V_{\beta}$. Since $(x, y)=\{x,\{x, y\}\}$ and by transitivity, $V_{\beta+3}$ has all functions from $\mathcal{F}$ to $\bigcup \mathcal{F}$, which includes the choice functions. And thus, $V_{\alpha}$ has choice functions for $\mathcal{F}$.

Now we have our model, somewhat. You see, we are constructing a model for ZFC but at the same time, we are going from objects that somehow satisfy all those axioms in the first place. This is a more complicated topic than we are making it sound with those proofs. We are just scratching the surface, in fact. If the reader wants more details, check [Kun83] for a better explanation.

Let's make one last definition. 
Definition B.3.12. Given a set A, let's define recursively, for each $n<\omega$, the following:

- $A_{0}=A$;

- $A_{n+1}=\bigcup A_{n}$.

Let's define the transitive closure of $A$ as the set $\operatorname{trcl}(A)=\bigcup\left\{A_{n}: n<\omega\right\}$. At last, given an infinite cardinal $\kappa$, let's define the set of hereditarily $<\kappa$ cardinality as

$$
H(\kappa)=\left\{x \in V_{\kappa}:|\operatorname{trcl}(x)|<\kappa\right\} .
$$

You see, the set $H(\kappa)$ represents all sets of cardinality less than $\kappa$ with elements of cardinality less than $\kappa$, all of which have elements of cardinality less than $\kappa$ and so on. Since $\epsilon$ is well founded, there are no infinite decreasing chains of elements in regards to $\epsilon$, that is, no infinite elements with $x_{0} \ni x_{1} \ni x_{2} \ni \cdots$ and as such, the definition we gave to the transitive closure is enough to cover all the elements of a given $x$ to determine if it is hereditarily of cardinality less than $\kappa$. So, for example, $H(\omega)$ is the set of all finite sets with finite elements and so on. $H\left(\omega_{1}\right)$ is the set of countable sets with countable elements and so on.

Proposition B.3.13. Given an infinite cardinal $\kappa$, the family $H(\kappa)$ is transitive, $\in$ is well founded in $H(\kappa)$ and $H(\kappa)$ models ZFC except for the power set axiom.

Proof. It's transitive because of it's definition. If $x \in X$ for some $X \in H(\kappa)$, then we have that $|\operatorname{trcl}(x)|<\kappa$ and thus $x \in H(\kappa) . \in$ is well founded due to the fact that $H(\kappa) \subset V_{\kappa}$. The rest of the axioms have the same demonstration due to transitivity and maybe few tweaks. In the case of the axiom of replacement, you can see that if for each $x \in S$, it's image $y$ is hereditarily of cardinality less than $\kappa$, then since $|S|<\kappa$, then the set of images is also of hereditarily of cardinality less than $\kappa$, thus it is in $H(\kappa)$.

Although it does not satisfies the power set axiom, it does satisfies it for some sets. For example, $H\left(\kappa^{+}\right)$satisfies the power set for all sets of size $\kappa$ and less. For everything that we will use, that will be enough. We will be using $H\left(\omega_{1}^{+}\right)$as our model of choice through the main work of this document, as we will be working with sets of size $\omega$ and $\omega_{1}$.

This is pretty much all we need for this work. There is another chapter on model theory, but it is self contained and we believe this appendix provides all the prior basis necessary to read it. 


\section{Bibliography}

[CK90] Chen Chung Chang e H Jerome Keisler. Model theory, volume 73. Elsevier, 1990. 69

[Dow88] Alan Dow. An introduction to applications of elementary submodels to topology. Em Topology Proc, volume 13, páginas 17-72, 1988. 13

[EFT13] H-D Ebbinghaus, Jörg Flum e Wolfgang Thomas. Mathematical logic. Springer Science \& Business Media, 2013. 39, 69

[Eps07] Richard L. Epstein. Classical mathematical logic. Princeton University Press, 2007. 39

[HJ99] Karel Hrbacek e Thomas Jech. Introduction to Set Theory, Revised and Expanded. Crc Press, 1999. 71

[Kro84] Leopold Kronecker. Näherungsweise ganzzahlige auflösung linearer gleichungen. 1884. 25

[Kun83] Kenneth Kunen. An introduction to independence proofs, volume 102 of studies in logic and the foundations of mathematics, 1983. 38, 71, 91

[Lar07] Paul B Larson. The nonstationary ideal in the p max extension. The Journal of Symbolic Logic, 72(1):138-158, 2007. 37

[Mar06] David Marker. Model theory: an introduction, volume 217. Springer Science \& Business Media, 2006. 69

[Men09] Elliott Mendelson. Introduction to mathematical logic. Chapman and Hall/CRC, 2009. 55,59

[Moo06] Justin Moore. A solution to the 1 space problem. Journal of the American Mathematical Society, 19(3):717-736, 2006. iii, v, 1, 19, 20, 25

[Rau06] Wolfgang Rautenberg. A concise introduction to mathematical logic. Springer, 2006. 39

[Tod87] Stevo Todorcevic. Partitioning pairs of countable ordinals. Acta Mathematica, 159(1):261-294, 1987. 1, 33, 37

[Tod07] Stevo Todorcevic. Walks on ordinals and their characteristics, volume 263. Springer Science \& Business Media, 2007. iii, v, 2, 7, 11, 12, 19 
[Tod10] Stevo Todorcevic. Walks on countable ordinals and selective ultrafilters. Bulletin (Académie serbe des sciences et des arts. Classe des sciences mathématiques et naturelles. Sciences mathématiques), páginas 65-78, 2010. 7

[Wee97] W.J.M. Weese. Discovering Modern Set Theory. II: Set-Theoretic Tools for Every Mathematician. Graduate studies in mathematics. American Mathematical Soc., 1997. 13

[Zen80] Phillip Zenor. Hereditary m-separability and the hereditary m-lindelöf property in product spaces and function spaces. Fundamenta Mathematicae, 106(3):175-180, 1980. 1, 25 